\title{
THERMO-ACOUSTIC VELOCITY COUPLING IN A SWIRL-STABILIZED GAS TURBINE MODEL COMBUSTOR
}

\author{
by \\ Vincent R. Caux-Brisebois
}

A thesis submitted in conformity with the requirements for the degree of Masters of Applied Science Graduate Department of Aerospace Engineering University of Toronto

Copyright @ 2013 by Vincent R. Caux-Brisebois 


\section{Abstract \\ THERMO-ACOUSTIC VELOCITY COUPLING IN A SWIRL-STABILIZED GAS TURBINE MODEL COMBUSTOR

\author{
Vincent R. Caux-Brisebois \\ Masters of Applied Science \\ Graduate Department of Aerospace Engineering \\ University of Toronto
}

2013

The research presented herein describes the coupling of acoustic and heat release fluctuations in a perfectly-premixed swirl-stabilized combustor by analysis of simultaneous high-repetition-rate laser diagnostics data. Nine cases are studied, varying the thermal power and the equivalence ratio. Proper orthogonal decomposition (POD) of the velocity data shows that cases with higher amplitude thermoacoustic oscillations have flow fields containing helical vortex cores (HVC); these cases are further analysed to determine the driving mechanisms of the oscillations. Flow and flame statistics are compiled as a function of both the phase in the thermoacoustic cycle and a phase representing the azimuthal position of the HVC relative to the measurement plane. These data are used to spatially map the thermoacoustic energy transfer field, as described by the Rayleigh integral. It is found that periodic deformations of the HVC cause large-scale flame motions, resulting in regions of positive and negative energy transfer. 


\section{Acknowledgements}

The fact that the cover page of this thesis reads Vincent $R$. Caux-Brisebois is a misleading proposition that the content of this work is the product of a single man's endeavour. Although nothing would please me more than adding the names of all those who made this possible, the imposed front-to-back limit of 100 pages would hardly allow the table of contents to be published. Nevertheless, I will try to show my appreciation to all those who made this possible in the short space that is available to me.

The second name that would go on the cover page of this document is without doubt Prof. Adam M. Steinberg's. In fact, if the degree of MASc. were not conditional to this dissertation, I would likely insist that it be written above mine. Prof. Steinberg is one of the few supervisors that I have known to always work with his door wide open. At any time during his long working hours, any student can walk in and ask any question knowing he or she will be well received, treated fairly, and perhaps most importantly, get the help they need. The work presented in this thesis would not have been possible without his valuable input, guidance, and hard work. Though I could go on about why Prof. Steinberg is a model supervisor, I have to cut this discussion short for the sake of space, and take this opportunity to thank the second reader of this dissertation, Prof. C. P. T. Groth, for taking some time out of his already-full schedule to review this work. Both Professors Steinberg and Groth were part of the Research Assessment Committee which guided me through my project, along with Professors Gotlieb, Gülder, Sampath and Sislian. For your time and advice, thank you!

Still at UTIAS, I cannot overlook the people who make this old building a great place to work. Though there is not enough space to mention the lot of you, I must include a special mention for the lab-mates who have put up with me a minimum of eight hours a day for the past year-and-a-half. Qiang, Michael, Maciej, Penelope, Jeff, and Hasnein have always provided a healthy balance of concentration and comical relief. To those of you who I have had lunch with, played tennis with, and partook with in countless activities outside of the great UTIAS walls (you know who you are), thank you! Let's not forget the members, past and present, of the Aerospace Students' Association (ASA), who always thought of different ways to keep us happy and entertained. A man by the name of Julius Cesar once said that providing a population with bread and games will keep them quiet and happy. While ASA strives against all odds to provide the games, 
they certainly provide the bread! Finally, the great UTIAS staff has always been helpful. Peter and Joan, keep up your fabulous attitudes, you make this place very special. Marta y Douglas, os quiero los dos y estaré aquí quatro años mas para compartir churros con vosotros!

To my fiends outside of UTIAS (not excluding people at UTIAS), the little engine inside me feeds off of your kindness, generosity, love, and humour. Thank you!

Finally, I have to mention my family. From the day I was born they have provided me with unwavering support, material and otherwise. While they have always looked out for my well-being, and they always offered advice in times of need, they have also provided me with the liberty to make my own path. It's not so much that without them I would never have gotten a Master's degree, it's that without them I wouldn't be the person I am today. Whatever good values I have today were transferred to me by my family. Maman, Papa, Philippe et Martin (urgghh), merci énormément pour votre support et votre amour. Non-plus devrais-je oublier la grande famille: Jeannot, Ben, Pierrette, Guylaine, Dan \& Dan, Pierre, Eva, Lyne, Marc, les cousins et les cousines. Je ne peus imaginer une meilleure famille. 


\section{Contents}

$\begin{array}{ll}\text { Abstract } & \text { ii }\end{array}$

Acknowledgements $\quad$ iv

Contents $\quad$ vii

List of Tables $\quad$ viii

List of Figures $\quad$ xi

1 Introduction $\quad 1$

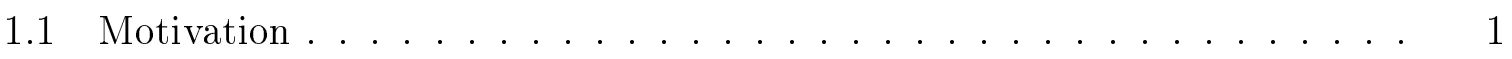

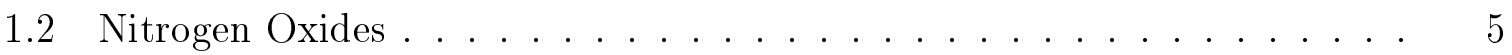

1.2.1 Effects and Legislation ................. 5

1.2.2 $\mathrm{NO}_{\mathrm{x}}$ and Combustion ................... 6

1.3 Thermo-Acoustic Coupling in Gas Turbine Combustors . . . . . . . . 8

1.4 Modelling Thermo-Acoustic Instabilities . . . . . . . . . . . . 11

1.4.1 Flame Transfer/Describing Functions . . . . . . . . . . . . 11

1.4.2 Flame Sheet Modelling ................ 13 
1.5 Swirl-stabilized flames in gas turbine combustors . . . . . . . . . . . 13

1.6 Optical Diagnostics . . . . . . . . . . . . . 16

1.6.1 Laser induced fluorescence . . . . . . . . . . . . 16

1.6.2 Chemiluminescence .................. 17

1.6.3 Particle image velocimetry . . . . . . . . . . . 17

1.6.4 High-repetition-rate diagnostics ............. 18

1.7 Review of Experimental Work in Swirl-Stabilized Combustors . . . . . . 18

1.8 Objectives . . . . . . . . . . . . . . . . . . . 19

2 Experiment and Basic Combustor Behaviour $\quad 21$

2.1 Combustor and Experiment . . . . . . . . . . . . 21

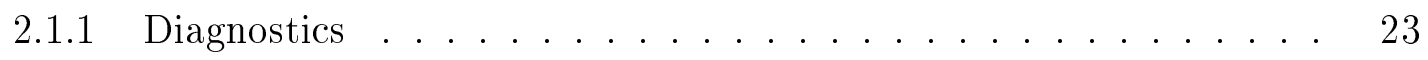

2.2 Overview of Flow-Flame Interactions in the Model Combustor . . . . . . 25

2.2.1 Fluid Dynamics . . . . . . . . . . . . 25

2.2.2 Flow-Flame Interactions within the Model Combustor . . . . . . 26

3 Analytical Techniques $\quad 29$

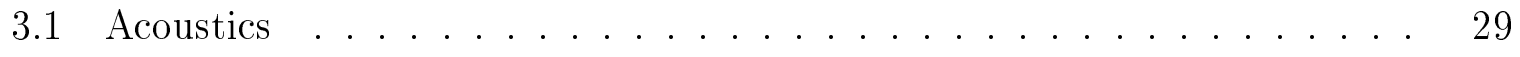

3.2 HVC Dynamics . . . . . . . . . . . . . . . . 30

3.3 Doubly Phase Resolved Analysis . . . . . . . . . . . . . . . 33

3.4 Flame Finding . . . . . . . . . . . . . . . . . 39

3.5 Flame Surface Density . . . . . . . . . . . . . . . 41

4 Results $\quad 44$ 
4.1 Thermo-Acoustic Energy Transfer . . . . . . . . . . . . . . . . 44

4.2 Flow-Flame Interactions Causing $\psi$ Distributions . . . . . . . . . . 47

4.2.1 Flame-HVC Interactions . . . . . . . . . . . . . 49

4.2.2 Origin of the $\omega_{\mathrm{z}}^{\mathrm{a}}$ structures . . . . . . . . . . 53

4.3 Phase Relationships . . . . . . . . . . . . . 60

4.3.1 Quantifying $\phi\langle S\rangle^{\mathrm{a}}-a \ldots \ldots . \ldots . \ldots . \ldots . \ldots . \ldots$

$\begin{array}{lll}5 & \text { Conclusion } & 67\end{array}$

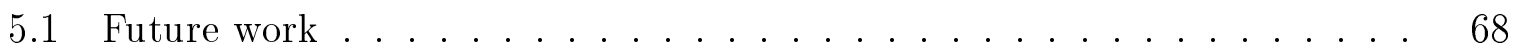

$\begin{array}{lr}\text { References } & 80\end{array}$ 


\section{List of Tables}

2.1 List of the different cases of the experiment and some important operating

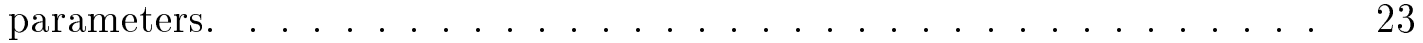

3.1 List of different cases and experiments showing dominant vortex type and associated frequency. . . . . . . . . . . . . . 34

4.1 Comparison of the mean axial flow velocity and the convective speed of the swirl wave. . . . . . . . . . . . . . . . . . . 59 


\section{List of Figures}

1.1 Schematic of a Rich-burn/Quick-mix/Lean-burn (RQL) combustor. . . . 3

1.2 Temperature dependence of the forward rate coefficient associated with the Zeldovich mechanism. . . . . . . . . . . . . . 7

1.3 Limit cycle amplitude (point A), resulting from arbitrary thermo-acoustic energy transfer and damping. . . . . . . . . . . . . . 10

1.4 Schematic of a typical swirling flow field near a confined expansion. . . . 14

1.5 Flame front wrinkling by a vortex. . . . . . . . . . . . 15

2.1 Schematic of the Turbomeca S. A. model combustor. . . . . . . . . 22

2.2 Layout of PLIF and s-PIV systems. . . . . . . . . . . . . . 24

2.3 Planar signature of a toroidal vortex . . . . . . . . . . 27

2.4 Instantaneous time sequence of swirling strength showing the planar signature of an HVC. ....................... 27

2.5 Time sequence showing the interactions between the HVC and heat release. 28

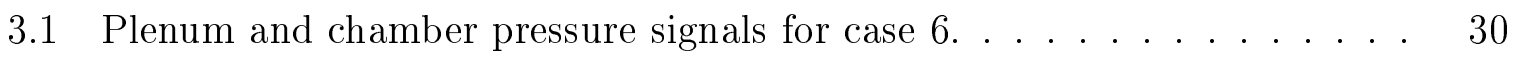

3.2 Acoustic frequency spectra of three different cases. . . . . . . . . . . . . 31

3.3 Swirl field POD reconstruction showing the planar signature of an HVC. 32

3.4 Signal burst of $a_{1}(t)$, corresponding to a helical mode. . . . . . . . . . 33 
3.5 Frequency spectra of $a_{1}(t)$ for a helical mode. . . . . . . . . . . . 34

3.6 Measurement decomposed into a long time average, phase, and random

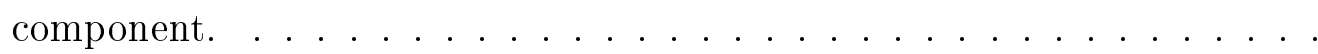

3.7 Definition of phase numbers used for doubly phase resolved analysis throughout the research project. . . . . . . . . . . 37

3.8 Illustrated representation of the douple-phase-resolving algorithm. . . . . 37

3.9 Domain of doubly phase resolved statistics around a helical vortex. . . . 38

3.10 Typical OH distribution across a stoichiometric laminar flame front. . . . 39

3.11 Important scalar fields in flame finding algorithm . . . . . . . . . . 41

3.12 Hypothetical grid division of the PLIF measurement plane for calculation of the flame surface density. . . . . . . . . . . . . . . 42

4.1 Thermo-acoustic energy transfer as a function of pressure oscillation. . . 45

4.2 Volumetric thermo-acoustic energy transfer distribution for all cases containing an HVC. ..................... 46

4.3 Flame hole surface density variation over the acoustic cycle. . . . . . . 47

4.4 Net flame surface density as a function of the acoustic phase. . . . . . . 48

4.5 Flame surface density slices taken at $\phi_{\mathrm{h}}=4$ of case $6 \ldots \ldots$. . . . . 49

4.6 Evolution of the FSD fluctuations, velocity fluctuations, and vorticity fluctuations in the vicinity of the HVC for the loudest case. $\phi_{\mathrm{h}}=1 . \ldots 50$

4.7 Evolution of the FSD fluctuations, velocity fluctuations, and vorticity fluctuations in the vicinity of the HVC for the loudest case. $\phi_{\mathrm{h}}=7 . \ldots 51$

4.8 Evolution of the FSD fluctuations, velocity fluctuations, and vorticity fluctuations in the vicinity of the HVC for the quietest case. $\phi_{\mathrm{h}}=2 \ldots \ldots 54$

4.9 Illustration of the HVC's deformation over the acoustic cycle. . . . . . . 56 
4.10 Axial and radial displacement of the vortex intersection for Case 6 over the acoustic cycle. ...................... 57

4.11 Axial velocity wave $\left(\left\langle u_{\mathrm{y}}\right\rangle^{\mathrm{a}}\left(\phi_{\mathrm{a}}\right)\right)$ as a function of axial location at different

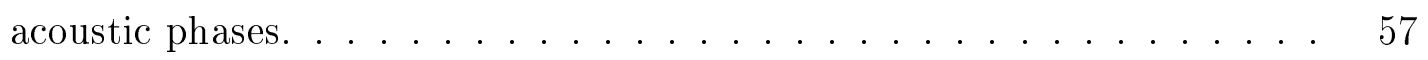

4.12 Comparison of the experimental vortex intersection displacement and the displacement that would result from convection by the $\langle a\rangle_{\mathrm{y}}^{\mathrm{a}}$ waves. . . . 58

4.13 Axial distribution of $\left\langle u_{\mathrm{y}}\right\rangle^{\mathrm{a}}$ and $\langle S\rangle^{\mathrm{a}}$ at $\phi_{\mathrm{a}=1}$ for case $6 . \ldots \ldots$

4.14 Swirl fluctuation plotted as a function of axial position and the acoustic

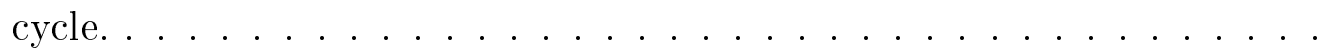

4.15 Fluctuations of the spatially integrated flame surface density $\left(\tilde{\Sigma}^{\mathrm{a}}\right)$ over the acoustic cycle for the quietest case (3), an intermediate case (5b), and the

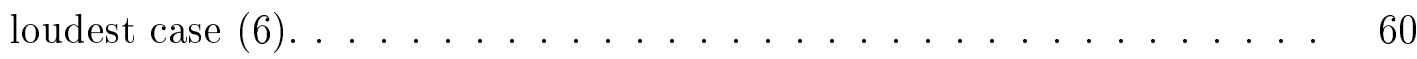

4.16 Phase relationship between the acoustic cycle, $\tilde{\Sigma}^{\mathrm{a}}$ and $\langle S\rangle^{\mathrm{a}}\left(y=0, \phi_{\mathrm{a}}\right) \ldots \quad 61$

4.17 Convective speed of the swirling strength wave as a function of the RMS pressure amplitude. . . . . . . . . . . . . 62

4.18 Simplified representation of the model combustor using a network of cylin-

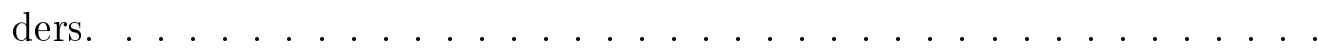

4.19 Spring-mass-damper model of the acoustic behaviour in the gas turbine

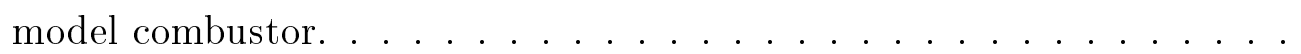

4.20 Free body diagram of the forces acting on $m_{\mathrm{s}}$ as it is accelerated in the positive $\xi$ direction. . . . . . . . . . . . . . . .

4.21 Phase lag of the reactant influx oscillations with respects to the chamber pressure as a function of acoustic forcing frequency. . . . . . . . . 66 


\section{Nomenclature}

\section{Symbols}

\begin{tabular}{|c|c|}
\hline$a$ & POD temporal coefficient \\
\hline$A$ & Area, Amplitude \\
\hline$b$ & Damping constant \\
\hline$f$ & Frequency \\
\hline$I_{0}$ & Flame stretch correction factor \\
\hline$k$ & Stiffness constant \\
\hline$k_{\mathrm{f}}$ & Forward rate of reaction \\
\hline 1 & length \\
\hline$m$ & mass \\
\hline$M$ & POD eigenmode \\
\hline$p$ & Pressure \\
\hline$P$ & Power \\
\hline$R$ & Radius \\
\hline $\mathbf{S}$ & Displacement \\
\hline$S$ & Swirl number \\
\hline$S_{\mathrm{L}}$ & Laminar flame speed \\
\hline$T$ & Temperature \\
\hline$m$ & Stiffness constant \\
\hline$\left(u_{\mathrm{x}}, u_{\mathrm{y}}, u_{\mathrm{z}}\right)$ & Cartesian velocity components \\
\hline$v$ & Convective velocity \\
\hline$V$ & Volume \\
\hline$(x, y, z)$ & Cartesian coordinates \\
\hline
\end{tabular}




\section{Greek}

$\begin{array}{ll}\gamma & \text { Ratio of specific heats } \\ \Theta & \text { Thermoacoustic energy transfer } \\ \lambda & \text { Eigenvalue } \\ \lambda_{\text {ci }} & \text { Swirling strength } \\ \rho & \text { Density } \\ \Sigma & \text { Flame Surface Density } \\ \Upsilon & \text { Thermo-acoustic damping } \\ \phi & \text { Phase, diameter } \\ \psi & \text { Local doubly-phase resolve thermoacoustic energy transfer } \\ \Psi & \text { Net doubly-phase resolved thermoacoustic energy transfer } \\ \omega & \text { Vorticity, angular velocity }\end{array}$

\section{Subscript}

ac Acoustic properties

$b \quad$ Burner properties

c Regarding a centroid

ch Regarding the combustion chamber

$f \quad$ Flame properties

$h$ Regarding the helical vortex core

$p l \quad$ Plenum properties

$s \quad$ Regarding the swirler

th Thermal properties

$v \quad$ Properties of a Vortex

\section{Superscript}

a Signifies the doubly phase resolved fluctuation over the acoustic cycle of a variable

ah Doubly phase resolved component of the associated variable

$t \quad$ Turbulent fluctuation of a variable

\section{Mathematical}

(:) Temporal derivative

$(\cdot)^{\prime} \quad$ Fluctuation

$(\cdot)^{\prime \prime} \quad$ Root-mean-square 
$(\sim) \quad$ Spatially integrated doubly phase resolved

$\langle\cdot\rangle \quad$ Radially and azimuthally integrated component

$\{\cdot\} \quad$ Radially and axially integrated component

\section{Acronyms}

CRZ Central recirculation zone

FDF Flame describing function

FHSD Flame hole surface density

FSD Flame surface density

FTF Flame transfer function

GT Gas turbine

HVC Helical vortex core

ICR Inner coupling region

LES Large eddy simulation

LIF Laser induced fluorescence

MC Model combustor

OCR Outer coupling region

ORZ Outer recirculation zone

PIV Particle image velocimetry

PLIF Planar laser induced fluorescence

POD Proper orthogonal decomposition

PVC Precessing vortex core

RMS Root-mean-square

RQL Rich burn-Quick mix-Lean burn

S-PIV Stereoscopic particle image velocimetry

UHC Unburnt hydrocarbons 


\section{Chapter 1}

\section{Introduction}

\subsection{Motivation}

The gas turbine based jet engine was pioneered by the English scientist Frank Whittle and his German counterpart Hans Von Ohain. Initially slow and underfunded, development was accelerated by the need for ever-increasing performance of aircraft in both camps of the Second World War, during which jet-powered interceptors entered service in the RAF and the Luftwaffe. This marked a milestone in aviation history, as from then on increasingly reliable, powerful, and efficient gas turbine engines have went on to power all but the smallest heavier-than-air aircraft. These characteristics also make gas turbine engines a viable and widely used means of power generation for ships, trains, generators, and even tanks. The tremendous power of gas turbine engines is extracted from the energy released by combustion of a reactive mixture. This emits pollutants into the atmosphere, which contribute to global climate change and air pollution. In an effort to mitigate these effects, authorities are setting increasingly stringent emissions regulations on fossil-fuel based combustion devices, concentrating legislation on the emission of carbon dioxide $\left(\mathrm{CO}_{2}\right)$, unburned hydrocarbons (UHCs), carbon monoxide $(\mathrm{CO})$, and nitrogen oxides $\left(\mathrm{NO}_{\mathrm{x}}\right)[1,2]$. Carbon dioxide is a product of hydrocarbon combustion, and it's emission can be reduced by bettering the efficiency of engines or by carefully selecting their fuel so as to minimize carbon intensity, a measure of $\mathrm{CO}_{2}$ emissions over the whole life cycle of the fuel including harvesting, refinement, distribution, etc. UHC emissions can be mitigated by increasing the residence time of gases in the combustion chamber, and 
increasing pressure and temperature in the combustor so as to increase the likelihood that any given combustible material will indeed react in chamber. These same conditions tend to reduce $\mathrm{CO}$ production, which is an intermediate product of combustion. It is an unfortunate consequence of these conditions, however, that the rate of production of the nitrogen oxides is increased [1]. These species are known to cause surface ozone $\left(\mathrm{O}_{3}\right)$ degradation [3] and are responsible for smog around large cities. $\mathrm{NO}_{\mathrm{x}}$ emissions are also responsible for several health issues in humans.

The nitrogen oxides produced by gas turbine engines are mainly a product of the Zeldovich mechanism, the rate of reaction of which is highly (exponentially) temperature dependant (see Sec. 1.2). As a result, methods of mitigating $\mathrm{NO}_{\mathrm{x}}$ emissions have focused on reducing the temperature of combustion through the effect of equivalence ratio. Because lean flames have less chemical energy per unit mass of reacting mixture, and products from rich flames have a greater capacity to absorb heat (due to the heavier molecules), the temperature of combustion can be decreased by burning lean or rich. This effect has been utilized to great success in two promising $\mathrm{NO}_{\mathrm{x}}$ reduction strategies: lean combustion and Rich-burn/Quick-mix/Lean-burn (RQL). The latter was introduced by Mosier et al. in 1980 [4], and has since then been the subject of numerous studies to become a well-accepted and commercially available method of mitigating emissions of nitrogen oxides [5-12]. The combustion process in an RQL burner is shown in Fig. 1.1. First, fuel and air burn in a rich environment (dark blue) insuring low combustion temperatures and low $\mathrm{NO}_{\mathrm{x}}$ formation. The products of this rich combustion contain partially-oxidized and partially-pyrolyzed reactants that must be burned before they reach the exhaust. Dilution air is added to reduce the equivalence ratio so that these unburned products may react in the lean zone (light blue). Note that the mixing must happen rapidly so that the equivalence ratio drops quickly from $\phi>1$ to $\phi<1$. For this to happen, the mixture's equivalence ratio must necessarily go through stoichiometric, temporarily increasing the temperature of combustion and the rate of production of $\mathrm{NO}_{\mathrm{x}}$. To minimize the time during which these conditions occur, mixing must be maximized and therefore the moniker "quick-mix" is used to highlight the importance of this requirement. Examining the RQL strategy closely, one may ask himself why is rich combustion necessary in the RQL scheme? Why not simply burn lean? Indeed, rich combustion is not necessary if the objective is solely to reduce $\mathrm{NO}_{\mathrm{x}}$ emissions. In fact, lean combustion alone usually produces less $\mathrm{NO}_{\mathrm{x}}$ than the RQL scheme, as combustion temperatures remain 
low throughout the combustor (there is no mixing zone in which the equivalence ratio is near unity) [13]. This makes lean combustion a more suitable candidate to address the increasingly stringent $\mathrm{NO}_{\mathrm{x}}$ emissions targets. However, lean combustors have shown to be susceptible to harmful high-amplitude self-excited pressure and heat release oscillations resulting from thermo-acoustic instability. Though other challenges arise from the use of lean combustors, such as increased UHC and CO emissions, flame blow-off, and an increased possibility of flashback, thermo-acoustic instabilities are the least understood of the challenges facing lean combustor technology. These instabilities result from the coupling of acoustic and heat release oscillations via some feedback mechanism, and the potentially high amplitude oscillations that result may lead to increased thermal and mechanical fatigue.

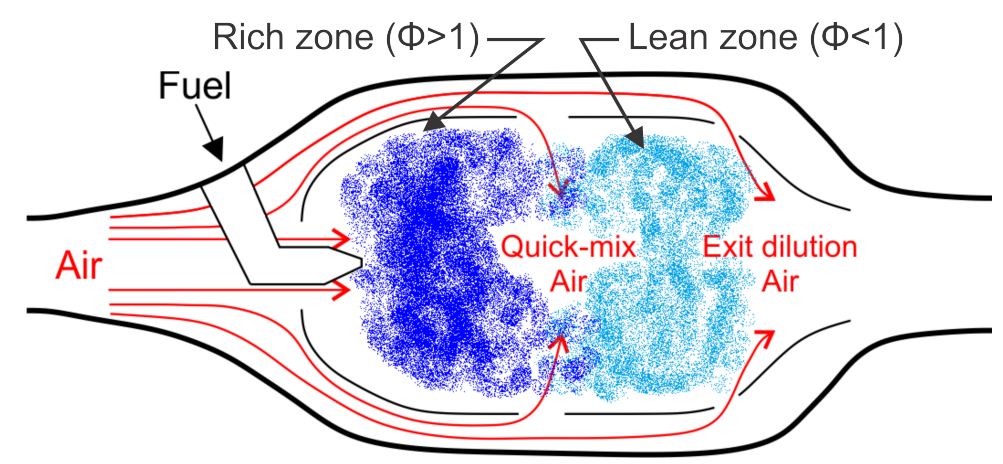

Figure 1.1: Schematic of a Rich-burn/Quick-mix/Lean-burn (RQL) combustor.

Various control strategies have been devised in an attempt to alleviate thermo-acoustic oscillations [14-17]. The different control strategies have had a limited success over a wide range of operating conditions due to the difficulties in modelling the thermo-acoustic behaviour of complex combustion systems. A common method of expressing a given combustor's thermo-acoustic response is the Flame Transfer Function (FTF), and more recently, the Flame Describing Function (FDF), which is a non-linear equivalent of the FTF that allows higher amplitude perturbations to be considered. FT/DFs are obtained for a given combustor geometry by operating the system over a range of conditions and measuring its response, at each condition, to acoustic forcing through a range of frequencies and amplitudes. The acoustic response is then expressed as a gain and a phase shift, which can be used to predict the system's response [18-21]. However, the requirement for experimental testing over a wide range of conditions makes FT/DFs a non-predictive tool, which can hardly be used for design applications. 
Flame sheet modeling has attempted to eliminate the need for experimental testing by modelling the flame's response to acoustic oscillations based on physical processes such as flow-flame interactions [22]. However, due to the complex nature of these interactions, such models have only yet been successful in predicting the acoustic response of simple flame geometries [23, 24], as the nature of the complex interactions between flow, flame, and acoustics remains ill-understood for flames relevant to gas turbine combustors. Although many flow and combustion processes that may potentially lead to coupling have already been identified [25], understanding how and when they couple with the acoustic field in the complex flow fields employed in today's combustors is a difficult task. High fidelity simulation of these mechanisms requires resolution of the finer time and length scales, and therefore simulation of thermo-acoustically coupled flames in the highly turbulent flow fields of technologically relevant combustor geometries is prohibitively expensive due to high computational costs. As a result, much effort has been expended into understanding thermo-acoustic coupling experimentally.

The application of modern laser-based diagnostics to combustion devices has provided much insight into the processes leading to thermo-acoustic instabilities. While investigations of simple flame geometries [26-34] have provided a detailed understanding of critical fundamental combustion processes, reduced optical access and high costs have limited similar studies in flame geometries relevant to gas turbine engines. In order to study these geometries, the combustion group at the German Aerospace Center (DLR) have devised a model combustor (MC). Based on an actual gas turbine engine's swirlstabilized combustor, the DLR burner reproduces the complex flow conditions desired while providing optical access for laser-based diagnostic techniques. Past research has shown that swirl-induced vortex breakdown lead to the formation of a central recirculation zone and large-scale helical vortices that interacted with the flame periodically, creating fluctuations in heat release $[35,36]$. While this research has clearly demonstrated the importance of the helical vortex in thermo-acoustic coupling, the mechanism through which the acoustic field coupled with heat release via the helical vortex remains unknown. This thesis aims to show the relationship between the acoustics, the velocity field, and the heat release oscillations that lead to thermo-acoustic coupling through analysis of previously collected experimental data from high-repetition rate laser diagnostics in a swirl-stabilized model combustor. Coupling between the acoustic and velocity fields will be quantified, and the coupling mechanism will be identified from flow-flame 
interactions.

\subsection{Nitrogen Oxides}

\subsubsection{Effects and Legislation}

Regulating agencies are placing an increased focus on $\mathrm{NO}_{\mathrm{x}}$ emissions due to the harmful effects that the molecules have on human health and the planet's climate. Direct consequences of high concentration $\mathrm{NO}_{\mathrm{x}}$ inhalation include the risk of acute lung injury and death [37]. Indirectly, $\mathrm{NO}_{\mathrm{x}}$ affects human health by creating ozone $\left(\mathrm{O}_{3}\right)$, which is responsible for photochemical smog. Smog consists of small particulate matter of diameters less than $2.5 \mu \mathrm{m}$ (PM2.5), which have been associated to increased mortality over the course of four decades (1974-2009) [38]. In addition to smog, ozone is considered a greenhouse gas that contributes to global climate change. Ozone affect's the planetary climate by changing the energy balance between the sun, the earth, and outer space via an increased radiative forcing of the tropopause. It is estimated that increased ozone concentrations in the troposphere have caused a change in preindustrial-to-present radiative forcing of $0.35 \pm 0.15\left[\mathrm{~W} / \mathrm{m}^{2}\right]$, based on simulations with chemical models [39]. The ozone responsible for photochemical smog is produced by nitrogen dioxide through the following reaction mechanism:

$$
\begin{gathered}
\mathrm{NO}_{2}+\text { uvsunlight } \rightleftharpoons \mathrm{NO}+\mathrm{O} \\
\mathrm{O}+\mathrm{O}_{2}+\mathrm{M} \rightleftharpoons \mathrm{O}_{3}+\mathrm{M},
\end{gathered}
$$

where $\mathrm{M}$ is an arbitrary molecule needed to absorb some of the energy from the exothermic reaction of the oxygens. In turn, nitrogen dioxide $\left(\mathrm{NO}_{2}\right)$ is generally created by the oxidation of rapidly cooling nitric oxide (NO) once combustion products have been released to the atmosphere [40, 41]. Therefore, addressing the health issues and global climate change related to $\mathrm{NO}_{\mathrm{x}}$ emissions depends largely on reducing the amount of nitric oxide released into the atmosphere.

The National Aeronautics and Space Administration (NASA) and the International Civil Aviation Organization (ICAO) have set stringent $\mathrm{NO}_{\mathrm{x}}$ emission targets to minimize the public's exposure to these harmful gases. Because the pollutant's effect on human 
health is more directly observed when it is released at low altitude, both organizations have set stringent landing and take-off (LTO) $\mathrm{NO}_{\mathrm{x}}$ emissions targets. NASA is aiming to achieve more than $75 \%$ reduction by the year 2025 [42]. while the ICAO has remained slightly more conservative, aiming for $60 \%$ reduction by the year 2026 [43]. To mitigate climate change due to ozone formation at altitude, NASA has also set cruise $\mathrm{NO}_{\mathrm{x}}$ reduction goals at $80 \%$ by 2025 [44] while the ICAO aims to reduce $\mathrm{NO}_{\mathrm{x}}$ emissions by the same amount before the year 2020 [45]. These targets require significant decrease in $\mathrm{NO}_{\mathrm{x}}$ emissions, and achieving either of them requires a thorough understanding of how $\mathrm{NO}_{\mathrm{x}}$ is formed during combustion.

\subsection{2 $\mathrm{NO}_{\mathrm{x}}$ and Combustion}

Emission of nitrogen oxides from hydrocarbon fuel combustion occurs when the fuel or the oxidizer contains nitrogen [46]. Indeed, nitrogen gas $\left(\mathrm{N}_{2}\right)$ makes up approximately $71 \%$ of the earth's atmosphere, and therefore fuel-air reactions are prone to $\mathrm{NO}_{\mathrm{x}}$ emissions. Since it was noted earlier that nitrogen dioxide is produced by the oxydation of nitric oxide, this section concentrates on the formation of NO.

Nitric oxide is formed by three competing chemical mechanisms: the extended Zeldovich (thermal) mechanism, the Fenimore (prompt) mechanism, and the $\mathrm{N}_{2} \mathrm{O}-$ intermediate mechanism [47]. Correa [48] studied $\mathrm{NO}_{\mathrm{x}}$ formation in flames under different conditions, relevant to gas turbine combustors. Navier-Stokes simulations with detailed chemistry, composed of 43 species and 154 reactions, were post-processed to obtain sensitivity coefficients relating any species to any reaction. It was found that the thermal mechanism was the dominant reaction pathway in both stoichiometric and lean methane-air flames. Similar research by Drake et al. [49] studied the relative contribution of the three mechanisms via a detailed chemical kinetics model containing 46 species and 212 reversible reactions. The NO contributed by each mechanism was studied over a range of pressures, and it was found that the Zeldovich mechanism was the main contributor in all but the lowest pressure flames $(0.1 \mathrm{~atm})$. Hence, for the purpose of this discussion, emissions of nitric oxides are considered to be the result of the Zeldovich mechanism. 
This mechanism consists of three reactions:

$$
\begin{gathered}
\mathrm{O}+\mathrm{N}_{2} \rightleftharpoons \mathrm{NO}+\mathrm{N}, \\
\mathrm{N}+\mathrm{O}_{2} \rightleftharpoons \mathrm{NO}+\mathrm{O}, \text { and } \\
\mathrm{N}+\mathrm{OH} \rightleftharpoons \mathrm{NO}+\mathrm{H},
\end{gathered}
$$

and it is coupled to the fuel oxidation chemistry via the $\mathrm{O}_{2}, \mathrm{O}$, and $\mathrm{OH}$ species. If assumed, however, that NO concentrations are low throughout the combustion process, and that they only become significant after combustion is complete, then the two processes can be decoupled [48]. If the time scale of the above reactions are sufficiently long, $\mathrm{N}_{2}$, $\mathrm{O}_{2}, \mathrm{OH}$ and $\mathrm{O}$ can be assumed to be in thermal equilibrium, while $\mathrm{N}$ concentrations are assumed to have reached steady-state. This means that Eq. 1.4 and 1.5 do not contribute to NO formation, thus the rate of production of NO may be expressed as [46]

$$
\frac{\mathrm{d}[\mathrm{NO}]}{\mathrm{d} t}=2 k_{\mathrm{f}}[O]_{\mathrm{eq}}\left[N_{2}\right]_{\mathrm{eq}}
$$

where $[x]_{\mathrm{eq}}$ is the molar equilibrium concentration of species $x$, and $k_{\mathrm{f}}$ is the forward rate coefficient associated to reaction 1.3. The latter may be expressed as a function of temperature $(\mathrm{T})$ via a semi-empirical Arrhenius type function:

$$
k_{\mathrm{f}}=1.8 \cdot 10^{11} \exp [-38370 / T(K)] \quad\left[\mathrm{m}^{3} / \mathrm{mol} \cdot \mathrm{s}\right] .
$$

Equation 1.7 shows the strong temperature dependence of the rate coefficient, which is plotted in Fig. 1.2.

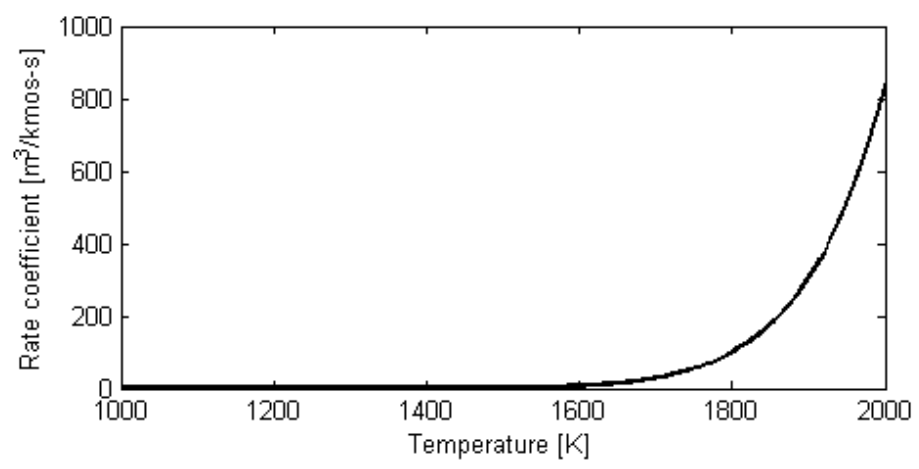

Figure 1.2: Temperature dependence of the forward rate coefficient associated with the Zeldovich mechanism. The rate increases exponentially with temperature. 
This implies that the NO formation of a given flame, largely dictated by the forward rate coefficient plotted in Fig. 1.2, is highly dependent on the temperature of the reacting gases. It follows that the NO emissions of a flame may be reduced by lowering the flame's temperature. As stated previously, one effective means of doing this is by adjusting the equivalence ratio - the ratio of fuel to air that composes the reacting mixture. While the global equivalence ratio of a non-premixed flame is defined as the fuel and oxidizer mass flow rate quotient, the local equivalence ratio of the combusting mixture cannot be controlled, as it depends of the flame geometry, flow field, and diffusive characteristics of the species. Hence, the rest of this thesis treats premixed combustion only. Heat release is diminished with low or high equivalence ratios, as less fuel reacts in lean mixtures and rich mixtures increase the specific heat of the mixture. This increase in heat capacity is a result of the fuel generally being composed of heavier and more complex molecules than the oxidizer. These heavier, more complex, molecules can absorb more energy because of their increased weight and number of vibrational modes. This provides an efficient, and easy way to decrease $\mathrm{NO}_{\mathrm{x}}$ emissions. Unfortunately, rich combustion introduces other pollutant emissions, such as unburned hydrocarbons and carbon monoxide. This has lead to great interest in lean combustion technology as a preferred strategy to mitigate $\mathrm{NO}_{\mathrm{x}}$ formation in gas turbine combustors. Unfortunately, these combustors have shown to be susceptible to coupled, high amplitude, pressure and heat release oscillations resulting form thermo-acoustic instabilities.

\subsection{Thermo-Acoustic Coupling in Gas Turbine Com- bustors}

Thermo-acoustic coupling is the result of natural acoustic modes of the combustor causing, through some mechanism, changes in the rate of heat release. If this heat release in turn causes the acoustic perturbation to be amplified, the thermo-acoustic coupling leads to instability in the system. Instability may lead to excessively high amplitude acoustic and thermal waves, causing increased fatigue loads on numerous engine components and possible flame blow-out, flashback, as well as reduced combustion efficiency. Thus, thermo-acoustic instabilities are extremely undesirable. Combustors found in gas turbine engines tend to be more susceptible to these instabilities than other combustion devices 
for two principal reasons [51]:

1 The energy required to drive unsteady motions represents an exceedingly small fraction of the heat released by combustion.

2 Combustion chambers are almost entirely closed and the internal processes tending to attenuate unsteady motions are weak.

The first attribute is best perceived through an example, such as given by Candel [25]. Given a mean chamber pressure of $10 \mathrm{MPa}$ and a mean power density of $E_{\mathrm{c}}=50 \mathrm{GW} \mathrm{m}^{-3}$, a $10 \%$ rms pressure disturbance may develop following an energy perturbation of no more than $E_{\mathrm{p}}=0.1 \mathrm{MW} \mathrm{m}^{-3}$. This represents a minute fraction of the total energy: $E_{\mathrm{p}} / E_{c}=10^{-6}$.

The second remark states that gas turbine combustors generally offer low damping of thermo-acoustic instabilities, as much of the unsteady energy cannot be dissipated to the surroundings because of the chamber walls. The amplitude of pressure and heat release oscillations will therefore increase until the rate of energy transferred into the acoustic field, $\Theta$, is equal to the rate of energy transmitted and dissipated by the system, $\Upsilon$. Though this condition is necessary for thermo-acoustic stability, it is not sufficient. The rate of increase of $\Upsilon$ must also be greater than the rate of increase of $\Theta$ for acoustic oscillations to stabilize [52]. Mathematically, the two conditions are

$$
\begin{aligned}
\Theta & =\Upsilon \text {, and } \\
\frac{\mathrm{d} \Theta}{\mathrm{d} p^{\prime \prime}} & \leq \frac{\mathrm{d} \Upsilon}{\mathrm{d} p^{\prime \prime}},
\end{aligned}
$$

where $p^{\prime \prime}$ is the root-mean-square of the pressure oscillations. This is best understood with the help of an illustration, such as Fig. 1.3. If the combustor begins operating with no pressure oscillations $\left(p^{\prime \prime}=0\right)$, a small, infinitesimal perturbation would mean there is more energy going into the acoustic field than dissipated or transferred out from the boundaries, as $\mathrm{d} \Theta /\left.\mathrm{d} p^{\prime \prime}\right|_{p^{\prime \prime}=0} \geq \mathrm{d} \Upsilon /\left.\mathrm{d} p^{\prime \prime}\right|_{p^{\prime \prime}=0}$. The oscillation amplitudes would continue to increase until they reach point $\mathrm{A}$, at which time they would stabilize. If the amplitude of the pressure oscillations would then decrease momentarily, the combustor would operate at a point just left of point A, where the instability would bring operation 
back to point A. Conversely, a positive pressure oscillation amplitude perturbation would bring the combustor to operate just right of point A. There, damping processes are more important than the driving ones and so the oscillation amplitude would gradually move back to point $\mathrm{A}$.

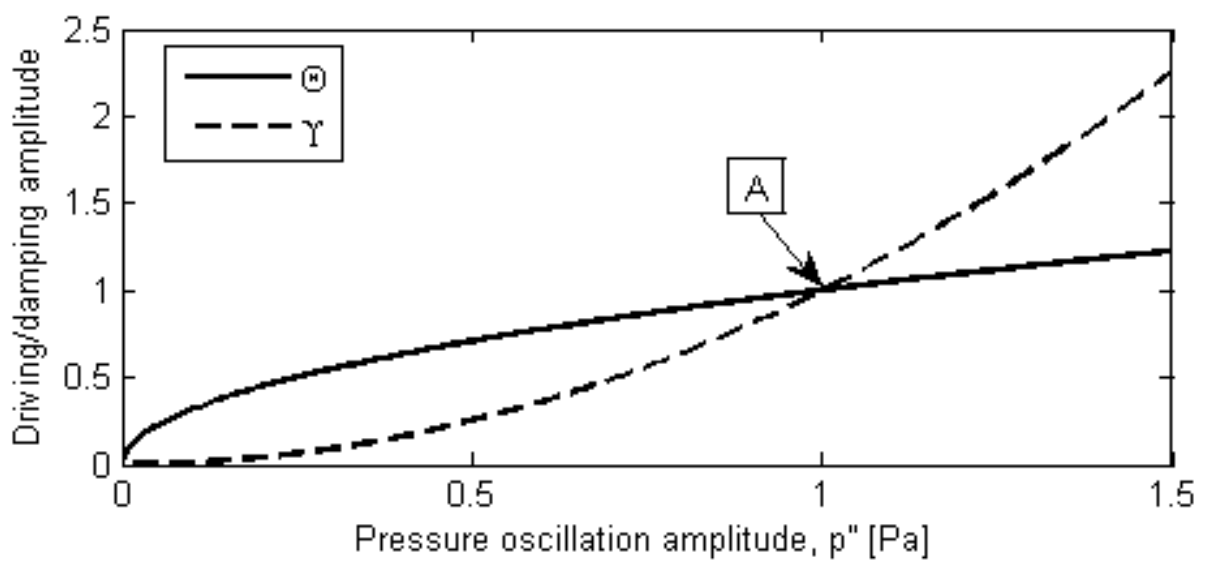

Figure 1.3: Limit cycle amplitude (point A), resulting from arbitrary thermo-acoustic energy transfer and damping. The conditions that describe the limit cycle amplitude are given by Eq. 1.8 and 1.9.

Damping processes such as mechanical hysteresis are difficult to quantify. $\Theta$, however, may be evaluated as a function of pressure $(p)$ and volumetric heat release $(\dot{q})$ via the Rayleigh integral,

$$
\Theta=\frac{\gamma-1}{\gamma} \frac{1}{\bar{p}} \int_{\mathrm{V}} \int_{\mathrm{t}} p^{\prime}(\mathbf{x}, t) \dot{q}^{\prime}(\mathbf{x}, t) \mathrm{d} t \mathrm{~d} \mathbf{x},
$$

where $\gamma$ is the ratio of specific heats and $\bar{p}$ is the mean pressure. The integrals are taken over any given time and volume over which the thermo-acoustic energy transfer is of interest. This relation states that if heat is generated at the moment of highest pressure or absorbed at the moment of lowest pressure, then the oscillations are excited. Conversely, if there is greater-than-average heat release at the moment of lowest pressure or lower-than-average heat release at the moment of highest pressure, then the oscillations are damped. In other words, the system tends to be unstable if the phase shift between heat release and pressure oscillations is less than 90 degrees. 
Thermo-acoustic instabilities are difficult to predict due to the complex physical phenomena involved: the detailed chemical reactions responsible for heat release, turbulent flow, and acoustics all are complicated when taken individually. When combined, general low-order prediction is not feasible [25]. Qualitatively, the instabilities are also hard to predict because of a lack of understanding of the underlying processes.

\subsection{Modelling Thermo-Acoustic Instabilities}

The interest in modelling thermo-acoustic instabilities comes from our desire to avoid them. Because of the harmful effects of large-amplitude pressure and heat release oscillations, gas turbine engine designers try to avoid thermo-acoustically unstable geometries and flow conditions during the design process, which requires predictive tools. If instabilities are still found in the finished combustor, control of the high-amplitude oscillations may be attempted. To avoid unstable combustion, it is necessary to understand the given combustor geometry's behaviour, and to this end Flame Transfer Functions (FTFs) and their non-linear cousins, the Flame Describing Functions (FDFs), were developed.

\subsubsection{Flame Transfer/Describing Functions}

Flame transfer/describing functions make use of heat release and velocity measurements to relate the acoustically-induced velocity perturbations to those of the heat release. Generally, a given flame is operated under many different conditions while, at each condition, it is excited acoustically over a range of frequencies. The flame's response is measured and the FT/DFs are derived from the velocity and heat release measurements. Mathematically, the FT/DFs are written as [52]

$$
\frac{Q^{\prime}}{\bar{Q}}=T(\omega, A) \frac{u^{\prime}}{\bar{u}},
$$

where $T$ is the transfer function, $\omega$ is the angular frequency of the velocity perturbations, and $A=\frac{\left|u^{\prime}\right|}{\bar{u}}$ is their amplitude. While this transfer function approach is very general, as it can be used to predict heat release oscillations for flames of any shape, it has the disadvantage of being difficult to model. Typically, the FT/DF is established by experiments or through direct numerical simulation (for simple flame geometries), in 
which the flame is operated at several conditions and excited acoustically over a range of frequencies and amplitudes [54]. The velocity and heat release perturbations are measured and the FT/DF can be established with proper post-processing. Unstable modes in the frequency domain can be identified by looking at the gain of the transfer function. An unstable mode is one that grows in time, and therefore any frequency at which the transfer function is greater than zero represents an unstable mode [52]. Using an appropriate model for damping, the limit cycle amplitude of the combustor may be established from the growth rate of the unstable modes.

Common FTF analysis uses a combined acoustic model approach to identify eigenmodes and their growth rates [55, 56]. These can then be used in a linear stability analysis to identify unstable modes, though the linear nature of this analysis limits its application to low amplitude perturbations. Hence, oscillations at the limit cycle cannot be accounted for, as they are known to be dominated by non-linearities [57-60]. Recently, Palies et al. [61] elaborated the non-linear FDF approach and applied it to study the response of a confined swirl stabilized flame. The FDF was obtained experimentally and combined with an acoustic transfer matrix analysis to derive a non-linear dispersion relation which was dependent on the amplitude of the velocity perturbations. This relation was then used to find the frequency and growth rate of oscillatory modes as a function of the upstream velocity perturbation amplitude. The growth rates were then compared to the damping rates of the combustor, which were also obtained experimentally, to determine whether individual modes were stable or unstable. The limit cycle amplitude was also obtained from this analysis.

The necessity of costly simulations and/or experiments to establish the transfer function (TF) of a given burner makes the FT/DF approach unfeasible for practical design work. At each design iteration, the combustor's TF should be established through costly numerical or experimental means for its unstable modes to be identified before another design iteration can be attempted. To avoid these large expenses, efforts have been concentrated on trying to establish the TF from operating conditions and geometry-related parameters. 


\subsubsection{Flame Sheet Modelling}

One widely used [62-64] method of modelling the TF makes use of the kinematic Gequation, in which the flame is represented as an infinitely thin surface $G=G_{0}$ separating fresh reactants $G<G_{0}$ from combustion products $G>G_{0}$ [25]. The G-equation represents the motion of a flame front with normal velocity $S_{\mathrm{L}}$ in a convective velocity field $\mathbf{v}$ via

$$
\frac{\partial G}{\partial t}+\mathbf{v} \cdot \nabla G=S_{\mathrm{L}}|\nabla G|
$$

The flame's response is analysed by modelling velocity disturbances and, assuming a uniform rate of reaction, the resulting heat release rate fluctuations are calculated from the flame surface perturbations [65]. In general, solutions of this equations have been attempted using simplifying assumptions such as axi-symmetric, purely axial velocity disturbances [18, 62]. As a result, only relatively simple flame geometries such as laminar flames [18, 62, 66, 67], "V" flames, and conical flames [24] have been successfully modelled. However, the complex flow-fields in gas turbine combustors do not allow for such simplifying assumptions, as the flow may be highly three dimensional. Swirling flows, for example, commonly used to stabilize flames in gas turbine engines, have not been faithfully modelled by this approach [22, 68, 69]. Proper modelling of swirl-stabilized combustors therefore requires a better understanding of swirling flows, and how they behave in acoustic fields.

\subsection{Swirl-stabilized flames in gas turbine combustors}

Flame stabilization based on swirling flows is common in gas turbine combustors, including the model combustor studied in this thesis. Stability of combustion is ensured by recirculating gases, which convey combustion products back to the root of the flame. This type of flame stabilization is important for combustion in gas turbines because it increases the residence time in a combustor of given length. This reduces the overall weight of the combustor and, therefore, of the engine assembly. Swirl also increases mixing within the chamber, which increases the overall rate of combustion. Reduced amounts of unburned hydrocarbons (UHC) and soot are two benefits offered by this increased mixing [70].

A key element to the aforementioned attributes of swirl stabilized combustors is the 


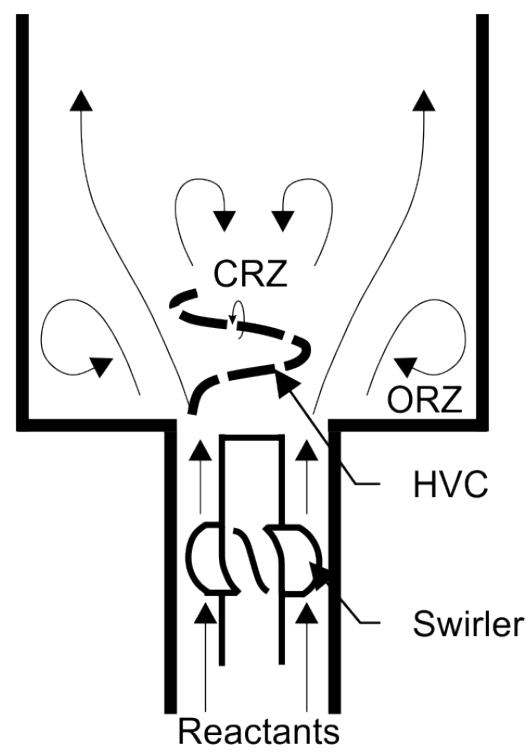

Figure 1.4: Schematic of a typical swirling flow field near a confined expansion.

formation of recirculation zones [71]. Due to swirl velocities, a centripetal force is required to balance the fluid's centrifugal acceleration. This centripetal force appears as a radial pressure gradient. As the flow is expanded through a nozzle, the tangential momentum decays, as does the radial pressure gradient. This downstream pressure equalization results in an adverse axial pressure gradient that, if strong enough, may cause flow reversal and the appearance of a central recirculation zone (CRZ). This CRZ transports hot products to the flame root and therefore is critical for flame stabilisation. Under some conditions, the velocity induced by the CRZ on the cyclone may cause it's axis to shift from the centreline. This configuration is unstable, and may cause a vortex to appear at the burner's nozzle. The vortex will precess around the burner axis, resulting in a precessing vortex core (PVC). The downstream portion of the PVC is wrapped into a helix by the swirling flow. The flow reversal responsible for both phenomena is only possible if the axial momentum is low with respect to the pressure gradient, which is itself a function of angular momentum. Hence, a critical parameter for the formation of CRZs and PVCs is the swirl number $S$, defined as the quotient of the angular momentum flux $\left(P_{\text {ang }}\right)$ and the product of the burner radius $\left(R_{\mathrm{b}}\right)$ with the linear momentum flux $\left(P_{\mathrm{x}}\right)$, such that

$$
S=\frac{P_{\mathrm{ang}}}{R_{\mathrm{b}} P_{\mathrm{x}}}=\frac{\int_{0}^{R_{\mathrm{b}}} \rho u w R_{\mathrm{b}}^{2} \mathrm{~d} r}{\int_{0}^{R_{\mathrm{b}}} \rho\left(u^{2}-\frac{1}{2} w^{2}\right) r \mathrm{~d} r}
$$

where $u$ and $w$ are the mean axial and swirl velocity components respectively [72]. 
Other parameters also affect the appearance of PVCs in combustion chambers. Of importance are the fuel injection method and chamber geometry. A PVC is less likely to form in radially confined areas as dissipation of angular momentum is attenuated. Recirculation zones are also likely to appear in the corners of confined swirl combustors. Figure 1.4, shows the important features of a confined swirl flow [51], including the CRZ, the PVC, and outer recirculation zones (ORZ).

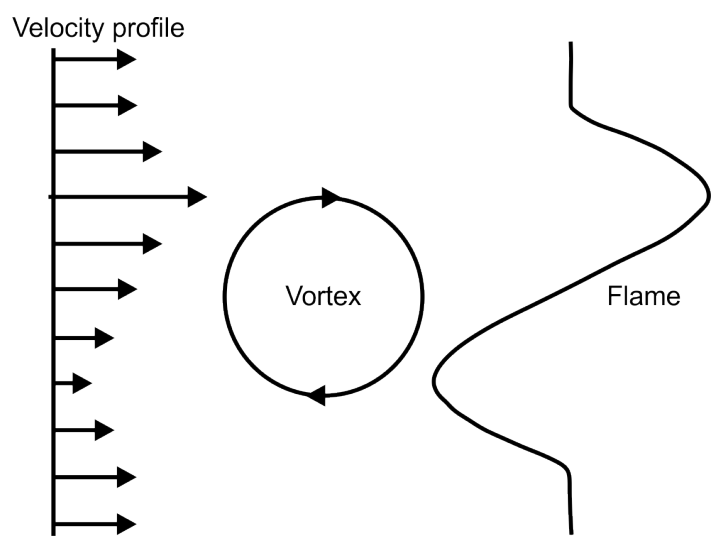

Figure 1.5: Flame front wrinkling by a vortex. Note the increased flame length, which leads to higher heat release in the region of the vortex.

The CRZ and PVC are important in combustion because of their interaction with the flame. CRZs, as well as secondary recirculation zones, provide the low velocities required for stable combustion. They also have the important role of anchoring the flame, by recirculating hot and reactive combustion species back to the flame root [72]. Also, Steinberg et al. [73] have shown that PVCs interact periodically with the flame surface to cause large scale flame corrugations. The mechanism by which a vortex may induce large scale flame corrugations is shown schematically in Fig. 1.5. These cause perturbations in heat release due to the variation in flame surface area, which may couple with the chamber acoustics. Due to the wide range of length and time scales associated to the flow, flame chemistry, and flow-flame interactions, direct numerical simulation of swirl-stabilized flames is prohibitively expensive. While Large Eddy Simulation (LES) of such flames has provided insight into their behaviour [74-76], they rely on subgrid models for turbulence, flame chemistry, and turbulence-flame interactions. These models remain an active area of research [77, 78], and they require validation with experimental 
results. It is therefore worthwhile to investigate the thermo-acoustic behaviour of swirlstabilized flames in a repeatable environment representative of swirl-stabilized gas turbine combustor. Such experiments often make use advanced laser diagnostics techniques, and therefore some of these will be reviewed in the coming section before moving on to a review of relevant experimental research in swirl-stabilized flames.

\subsection{Optical Diagnostics}

Optical diagnostics are often used in experimental combustion research to obtain velocity and chemical composition information of a fluid field. One reason for this is that optical techniques have the advantage of being non-intrusive. That is, optical methods do not perturb the fluid stream as do other methods such as hot wire anemometry, probes, and pitot-static tubes. This section will briefly describe the three commonly-used laser diagnostics techniques relevant to this project in sufficient detail to understand the advantages and limitations of the methods.

\subsubsection{Laser induced fluorescence}

Laser induced fluorescence (LIF) is a type of laser diagnostics used in fluid mechanics and combustion research to obtain information regarding distributions of particular species in a flow field. Other quantities, such as temperature, also can be measured using LIF. In LIF, the flow is irradiated with laser light that is tuned to a particular rovibronic transition of the species of interest [79]. When the laser radiation interacts with the target species, it can cause a transition to an excited state. This state is unstable, and the atom or molecule rapidly returns to a stable state, emitting light as it does so. Some of this emitted light is at a different wavelength than the laser light, and can be imaged by an intensified camera to determine the distribution of the emitting species. The method is sensitive and capable of high spatio-temporal resolutions which makes it an effective tool for measurements in turbulent combustion. As noted by Radi et al [80], many molecular species may be mapped with the technique. However, in combustion, one of the most commonly mapped species is the hydroxyl radical $(\mathrm{OH})$ because of its link to the flame front and its relatively high signal [81]. 


\subsubsection{Chemiluminescence}

Much like LIF, chemiluminescence is used to obtain information about the flame by imaging photons emitted as a molecule transitions from an excited state to a lower energy state. However, chemiluminescence differs from LIF in that no external source of energy (i.e. laser) is necessary to excite the molecule. Rather, the molecule is excited by collisions with its neighbours. A common example of chemiluminescence is the glowstick. In combustion, the blueish glow emitted from non-sooting flames is a result of chemiluminescence of the $\mathrm{CH}^{*}, \mathrm{OH}^{*}, \mathrm{CO}_{2}{ }^{*}$ and $\mathrm{C}_{2}{ }^{*}$ species [82]. Because molecular excitation is chemical reaction induced, photons emitted anywhere in the three dimensional field of view of the intensifying camera may be imaged. Thus, chemiluminescence measurements are line-of-sight integrated.

\subsubsection{Particle image velocimetry}

While LIF and chemiluminescence are powerful tools to obtain information on the flame, they provide little information on flow dynamics. For this reason, another diagnostics must be introduced: particle image velocimetry (PIV). PIV is a tool in experimental fluid dynamics that allows measurement of two or three component planar velocity fields. The method is performed by seeding a fluid flow with particles and observing their evolution in time so as to deduce the velocity vectors of these particles [83]. The flow at a given section is successively illuminated twice with a light sheet, most often created using a laser. The light scattered from the seeded particles is then imaged at two instants in time. For threecomponent measurements, the scattered light is imaged from two different angles in a stereoscopic configuration (s-PIV). The velocity may be approximated as $\mathbf{v}=\Delta \mathbf{x} / \Delta t$, where $\Delta \mathbf{x}$ is the displacement of particles and $\Delta t$ the time between images. Although individual particle tracking has been performed in the past [84], it is more common to find the velocity vectors statistically. To do this, the image plane is divided into interrogation cells composed of several pixels. The statistical displacement of the particles within each cell may then be determined by cross-correlation of the image sequence. 


\subsubsection{High-repetition-rate diagnostics}

Most laser-based diagnostics employed in the past have been used to acquire measurements at a rate of approximately $10 \mathrm{~Hz}$. This rate of data acquisition is insufficient to resolve thermo-acoustic processes, which typically happen at a few hundred Hertz. In order to temporally resolve these processes, acquisition rates on the order of the kilo Hertz $(\mathrm{kHz})$ are required, and have recently been available thanks to advances in laser and high speed camera technologies. These modern diagnostics will be used in the current thesis.

\subsection{Review of Experimental Work in Swirl-Stabilized Combustors}

Experimental analysis of swirl-stabilized flames and thermo-acoustic coupling is a wide field of study, for which Syred [71] provides a detailed review. This section will focus on research performed in a gas turbine model combustor (GTMC) derived from an industrial design by Turbomeca. Specially designed with laser diagnostics in mind, this burner bridges the gap between laboratory scale fundamental combustion studies and industrial-scale experiments, which are usually ill-suited to advanced diagnostics techniques. Furthermore, the geometry was selected as a target case by the International Workshop on Advanced Measurement Techniques and Computational Methods for Premixed and Partially Premixed Combustion, and it has been the subject of many experimental [85-88] and computational [89-91] studies. Here, experiments performed in two different versions of the Turbomeca-based model combustor will be reviewed. The first combustor (burner \#1) operated a non-premixed flame, in which non-swirling methane was injected between co-swirling jets of dry air. The second burner operated a premixed flame in which perfectly premixed fuel and dry air was fed to a plenum before going through the swirler into the combustion chamber $(\# 2)$.

Weigand et al. [92] and Meier et al. [93] studied the GTMC under a variety of partiallypremixed operating conditions in burner \#1. Using OH/CH PLIF, laser Doppler velocimetry, and Raman scattering, they found that the flame did not anchor on the fuel injector, but that it was lifted by a few milimeters, allowing for fuel the and air to partially mix prior to combustion. Combustion happened in thin layers, and interacted with 
the turbulent flow, which caused it to corrugate and extinguish. These flow-flame interactions were further studied by Sadanandan et al. [35] and Stöhr et al. [36], who used s-PIV and OH PLIF measurements to conclude that the helical vortex was a dominant structure in the flow field. This HVC precessed around the burner at a frequency different from the main acoustic frequency, and important interactions with the flame front were observed. To further elucidate these interactions, Boxx et al. [94] and Stöhr et al. [95] performed high-repetition-rate s-PIV, OH PLIF and $\mathrm{OH}^{*}$ chemiluminescence measurements at a rate of $5 \mathrm{kHz}$, allowing qualitative observation of flow-flame interactions through time sequences. The HVC was found to contribute to heat release oscillations by rolling up the flame, and via local extinctions. Possible auto-ignition events were also noted, though it was difficult to distinguish autoignition from through-plane, azimuthal convection of a quasi-stable flame. To address this problem, Steinberg et al. [73] statistically decoupled the azimuthal position of the HVC and the acoustic cycle by resolving the time-accurate data with respects to the acoustic and HVC phases. This process is detailed in Sec. 3.3. The HVC was found to deform axially over the acoustic cycle, and the deformations were not simultaneous everywhere along the span of the vortex. As it expanded and contracted, the HVC interacted with the flame front, causing periodic oscillations in heat release at the thermo-acoustic frequency. This created large regions of positive thermo-acoustic coupling which drove the instabilities.

Despite the insight that this research provides to the combustion community, it is unclear to what extent the HVC's interaction with the flame front are responsible for the heat release oscillations. This is due to the partially-premixed nature of the experiments, in which local oscillations in equivalence ratio may also largely affect the rate of heat release. To isolate the velocity coupling from equivalence ratio coupling, Steinberg et al. [96] used a modified version of the GTMC (burner \#2) to repeat the high-repetitionrate measurements of Boxx et al. [94] and Stöhr et al. [95] under perfectly premixed conditions. This thesis treats the analysis of this data.

\subsection{Objectives}

The remainder of this thesis is dedicated to the analysis of the data previously collected by Steinberg et al. [96] in a GTMC burning a perfectly premixed flame. This data, 
obtained at the German Aerospace Center (DLR), will be used to elucidate the role of the HVC-flame interactions in setting the thermo-acoustic behaviour of the combustor. The goal of this thesis is

1. To identify flow-flame interactions leading to positive and negative thermo-acoustic coupling in a gas turbine model combustor via the analysis of previously collected experimental data, and

2. to outline the mechanism responsible for coupling of the HVC dynamics, the heat release oscillations, and the acoustic oscillations.

The two objectives require that flow and flame interactions be characterized and that thermo-acoustic energy transfer be quantified. This in turn requires knowledge of the spatially resolved acoustic field and volumetric heat release throughout the acoustic cycle. To this end, the thesis is divided into four sections, namely experiment and combustor behaviour, analytical techniques, results, and conclusion. The coming section will present the experimental setup used to acquire the data analysed in this thesis, and give qualitative insight into the flow and flame dynamics in the model combustor. Some analytical tools necessary to interpret the experimental measurements are then laid out prior to treatment and analysis in the results section. Conclusions are then drawn and discussed in the final chapter. 


\section{Chapter 2}

\section{Experiment and Basic Combustor Behaviour}

The first section of this chapter will present a detailed description of the equipment used in the experiments, performed at DLR, including a description of the model combustor's geometry and the laser diagnostics used to acquire the data. Then, the fluid dynamics are detailed before discussing qualitative flow-flame interactions in the model combustor.

\subsection{Combustor and Experiment}

The gas turbine model combustor in which the data analysed in this thesis was obtained is shown in Fig. 2.1 [96]. Methane and dry air were metered using electromechanical mass flow controllers (Brooks) and then mixed in a commercial premixer. The upstream acoustic boundary condition was controlled approximately $0.15 \mathrm{~m}$ upstream of the plenum, where the mixture passed through a chocked orifice, the diameter of which was adjusted in accordance with the measured pressure to insure choked flow. The reactants then went to a plenum $(\phi=78 \mathrm{~mm})$ before being fed through a radial swirler composed of 12 swirl vanes. The inner surface of the swirler was a conical bluff body that directed the flow towards the nozzle $(\phi=27.85 \mathrm{~mm})$ and into the combustion chamber. Flow in the combustion chamber had a swirl number of approximately $\mathrm{S}=0.5$ [73]. The chamber had a square cross-section ( $85 \mathrm{~mm}$ x $85 \mathrm{~mm}$ x $114 \mathrm{~mm}$ ), and was delimited by four fused 
silica windows held in place by Inconel posts in the corners. This made the combustor optically accessible and suitable for optical diagnostics. The combustion products exited through a conical steal plate which joined the chamber to the exhaust tube $(\phi=40 \mathrm{~mm})$.

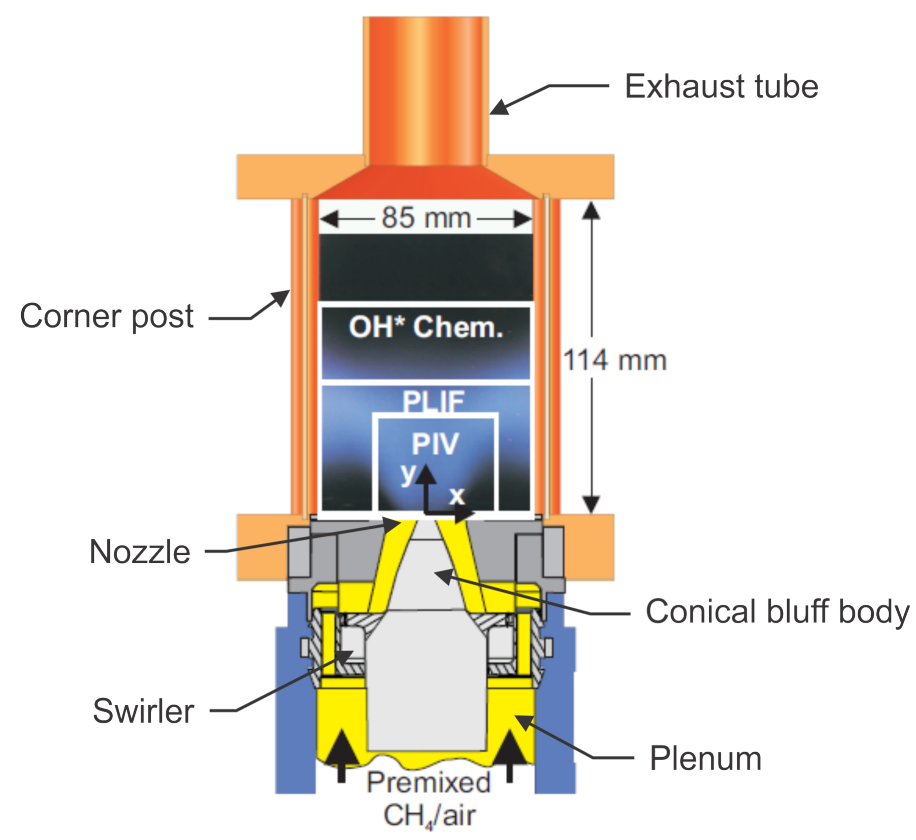

Figure 2.1: Schematic of the Turbomeca S. A. model combustor used in the experiments. Note the diagnostics field of views.

Measurements were acquired under nine different conditions, encompassing two distinct parameter variations. The first parameter study was at a constant equivalence ratio of $\phi=0.70$, with thermal powers between $P_{\mathrm{th}}=10-35 \mathrm{~kW}$ in increments of $5 \mathrm{~kW}$. The second parameter study was at a constant thermal power of $P_{\mathrm{th}}=30 \mathrm{~kW}$, with equivalence ratios between $\phi=0.65-0.80$ in increments of 0.05 . The different cases are listed in Table 2.1. Information about the flow field and flame dynamics was obtained by stereoscopic particle image velocimetry (S-PIV) and planar laser induced fluorescence (PLIF) of the $\mathrm{OH}$ radical, respectively, at a rate of $10 \mathrm{kHz}$. Additionally, acoustic data was gathered using calibrated microphone probes installed in a corner post, at a rate $50 \mathrm{kHz}$. 


\begin{tabular}{ccccccc} 
Case & $\phi$ & $P_{t h}[\mathrm{~kW}]$ & $\dot{m}_{\text {air }}[\mathrm{g} / \mathrm{s}]$ & $\dot{m}_{C_{H_{4}}}[\mathrm{~g} / \mathrm{s}]$ & $p^{\prime \prime}[\mathrm{Pa}]$ & Dominant flow structure \\
\hline & & & & & & \\
\hline 1 & 0.7 & 10 & 295 & 12 & 19.4 & Toroid \\
2 & 0.7 & 15 & 440 & 18 & 20.2 & Toroid \\
3 & 0.7 & 20 & 588 & 24 & 107.4 & HVC \\
4 & 0.7 & 25 & 733 & 30 & 74.4 & HVC \\
\hline $5 \mathrm{a}$ & 0.65 & 30 & 940 & 36 & 145.4 & HVC \\
$5 \mathrm{~b}$ & 0.7 & 30 & 880 & 36 & 149.1 & HVC \\
$5 \mathrm{c}$ & 0.75 & 30 & 824 & 36 & 177.2 & HVC \\
$5 \mathrm{~d}$ & 0.80 & 30 & 770 & 36 & 105.2 & Toroid \\
\hline 6 & 0.7 & 35 & 1020 & 42 & 181.9 & HVC
\end{tabular}

Table 2.1: List of the different cases of the experiment and corresponding equivalence ratio $\phi$, thermal power $P_{\text {th }}$, mass flow rate of air $\dot{m}_{\text {air }}$, mass flow rate of methane $\dot{m}_{C H_{4}}$, RMS pressure amplitude $p^{\prime \prime}$, and dominant flow structure (see Sec. 2.2.1).

\subsubsection{Diagnostics}

This section presents a brief overview of the optical diagnostics used during the experiments, as a detailed description of the diagnostics can be found in Ref. [96]. For an illustration of the experimental configuration in which the laser based diagnostics were used, see Fig. 2.2.

The S-PIV system used a high-repetition-rate, dual cavity, diode-pumped solid-state (DPSS) ND:YAG (Edge-wave, IS-6IIDE) laser for illumination. The $532 \mathrm{~nm}$ wavelength beam was formed into a $40 \mathrm{~mm}$ high sheet with approximately $1 \mathrm{~mm}$ full-width-at-halfmaximum (FWHM) using cylindrical lenses. Scattering from $\mathrm{TiO}_{2}$ seeding was imaged with two high-speed CMOS cameras (LaVision HSS 8) mounted equidistantly on both sides of the laser sheet. Both cameras were equipped with $100 \mathrm{~mm}$ focal length commercial lenses (Tokina) set to $\mathrm{f} / 5.6$. The $49 \times 38 \mathrm{~mm}$ field of view of the cameras was offset from the centreline of the burner due to optical restrictions imposed by the corner posts of the model combustor, covering a range of approximately $\mathrm{x}=-17 \mathrm{~mm}$ to $\mathrm{x}=32$ mm. The laser pulse separation was $5-15 \mu \mathrm{s}$. Vector fields were computed from particle images using the LaVision DaVis 7.2 software package. The resulting vector fields had a 
resolution and vector spacing of approximately $1 \mathrm{~mm}$ and $0.5 \mathrm{~mm}$, respectively.

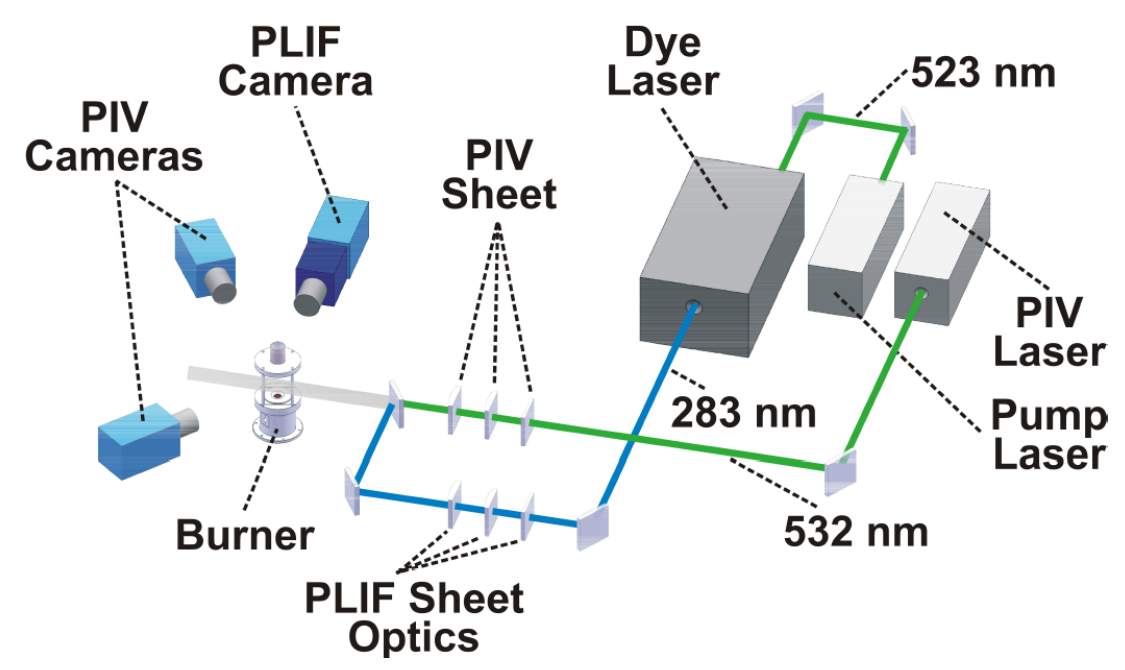

Figure 2.2: Layout of PLIF and s-PIV systems.

PLIF of the $\mathrm{OH}$ radical was performed by exciting the molecules at $283.2 \mathrm{~nm}$. The excitation came from a dye laser (Sirah Credo) pumped by a DPSS (Edgewave IS811-E) with an output of approximately $140 \mu \mathrm{J}$ per pulse and an average power of $1.4 \mathrm{~W}$. As for the s-PIV system, the laser beam was formed into a $40 \mathrm{~mm}$ high sheet using cylindrical lenses. The sheet was approximately $0.4 \mathrm{~mm}$ thick. The $\mathrm{OH}$ fluorescence was imaged at $310 \mathrm{~nm}$ using a CMOS camera (LaVision HSS 5) with an external, 2-stage, lens-coupled intensifier (LaVision HS-IRO) and a $45 \mathrm{~mm} \mathrm{f} / 1.8 \mathrm{UV}$ lens (Cerco). All PLIF images were filtered with a $0.4 \mathrm{~mm}$ Gaussian filter (same width as the sheet thickness) prior to post-processing in order to reduce high frequency noise.

OH* chemiluminescence was imaged using an identical configuration to that of the PLIF, with the exception of the intensifier gate time. Furthermore, since chemiluminescence does not rely on a source of excitation (laser), the field of view was not restricted by the hight of the laser sheet and measurements therefore had a square $(85 \times 85 \mathrm{~mm})$ field of view.

The s-PIV and PLIF laser sheets were superimposed using a dichroic mirror. To reduce the possibility of interference between the PLIF and s-PIV systems, the PLIF laser pulse was timed to go off between the two pulses of the s-PIV's pulse pairs. 


\subsection{Overview of Flow-Flame Interactions in the Model Combustor}

\subsubsection{Fluid Dynamics}

As a first step towards understanding flow-flame interactions in the model combustor, this section will discuss key properties of the flow field. In Sec. 1.7, it was noted that researchers had observed coherent structures that interacted with the flame periodically. This section will first discuss the identification of such flow structures from the laser diagnostics measurements, before elaborating on how they interacted with the flame within the GTMC studied here.

\section{Identification of Coherent Flow Structures}

Many methods have been proposed to identify vortices in fluid flows. In viscous flows, vortices are known to be associated with regions of high vorticity $(\nabla \times \mathbf{v})$. Thus, one of the most popular methods of vortex identification is to find peaks and valleys in the vorticity field. Vorticity, however, physically represents the angular velocity of a fluid particle and does not necessarily indicate the presence of vortices. Shear layers [97] and baroclinic stresses, for example, may also lead to peaks and valleys in the vorticity field. Vortices are also known to have low pressures at their core, and therefore a pressure minimum criterion has also been used as a means to locate them. This criterion presents its own set of problems, as source and sink flows also have a pressure minimum at the origin. Furthermore, the pressure field may be significantly perturbed in reacting flows.

Jeong \& Hussain [98] propose that a vortex core may be identified given two requirements:

1. A vortex core must have net vorticity and circulation.

2. The geometry of the identified vortex should be Galilean invariant. This means that the geometry should be the same in all inertial reference frames.

Mathematically, these conditions are insufficient to provide a single solution, and there- 
fore several methods of identifying vortices exist [99]. Many of these make the use of velocity gradient invariants to identify vortex tubes, thereby discriminating tubular vortices from vortex sheets.

None of the previously mentioned methods have been proven to achieve better performance than any other [99]. Rather, the choice of a method for identifying vortices depends on the investigator's concept of a vortex. For this work, the $\lambda_{\text {ci }}$ criterion, also known as the swirling strength criterion, will be used. It is mathematically defined as the imaginary component of the eigenvalues of the velocity gradient tensor:

$$
\lambda_{\mathrm{ci}}=\Im(\operatorname{EigenVal}(\nabla \mathbf{v}))
$$

The swirling strength criterion has the advantage of identifying the shape, as well as the intensity of a vortex core.

\section{Large Scale Coherent Structures in the Model Combustor}

The swirling strength fields were computed for every experimental case, and two different coherent flow structures were found to dominate the flow fields, depending on the conditions; loud flames contained helical vortex cores (HVCs) while quieter flames contained periodic toroidal vortex shedding from the burner nozzle. This is illustrated in Figs. 2.3 and 2.4, which show instantaneous swirling strength fields derived from measurements of a representative quiet and loud case, respectively. In Fig. 2.3, the toroids appear in the laser sheet as counter-rotating vortices that propagate symmetrically from the burner nozzle, whereas the helical vortex has a planar signature consisting of counter-rotating vortices in a staggered configuration (see Fig. 2.4). Since the focus of this work is mechanisms exciting thermoacoustic oscillations, and since the HVC dominated cases were louder than those with toroidal vortices, this work will focus on cases with HVCs; viz. Cases 3, 4, 5a, 5b, and 6. The dominant flow structures of each case is listed in Table 2.1 .

\subsubsection{Flow-Flame Interactions within the Model Combustor}

Now that the coherent structures in the flow field have been identified, the flow-flame interactions may be investigated. Flow-flame interactions in the model combustor were 


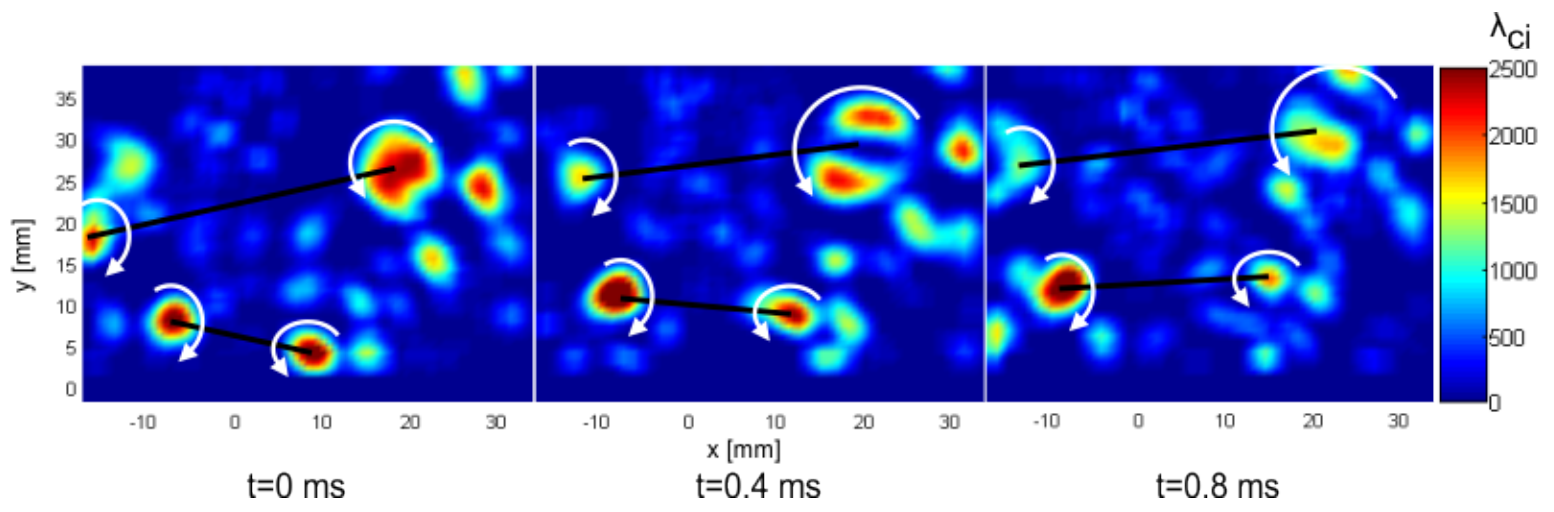

Figure 2.3: Instantaneous time sequence of swirling strength showing the planar signature of toroidal vortex shedding from the burner nozzle.

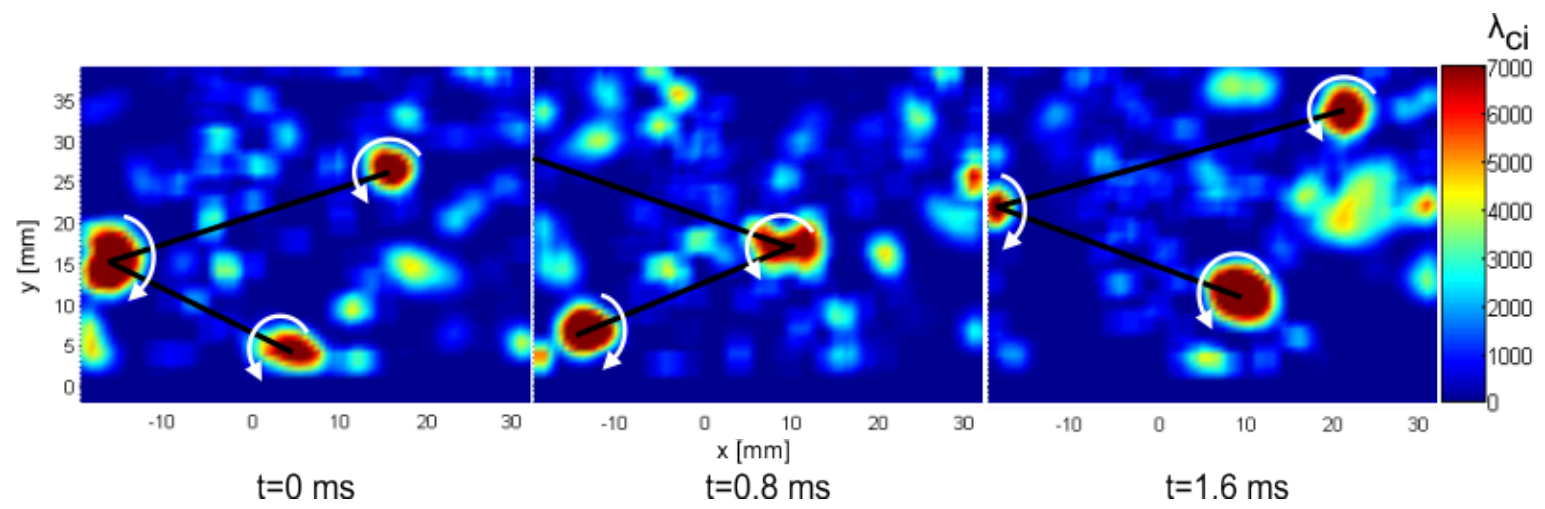

Figure 2.4: Instantaneous time sequence of swirling strength showing the planar signature of an HVC.

studied by Steinberg et al. and are reported in Ref. [96]. In the experimental cases with HVCs, the flame was found to be greatly affected by the vortex, showing largescale flame wrinkling induced by the HVC. This is illustrated in the time sequence of Fig. 2.5, which shows the swirling strength fields and OH PLIF fields at corresponding times. For comparison, an isocontour of the OH PLIF field is overlaid on the swirling strength field, appearing as a white line, which is an approximate indication of the flame topography. At $t=0 \mathrm{~ms}$, the flame was roughly anchored around the recirculation zone, and the HVC intersected the measurement plane at two different locations (numbered 1 and 2). The outward flow induced by the HVC corrugated the flame near the root, creating an upside-down "T" shaped flame. After $0.4 \mathrm{~ms}$ of time elapsed, vortex 1 had decreased in strength due to viscous dissipation or baroclinic stresses, leaving vortex 2 to have a greater influence on the flame. This moved the flame to the left as it wrapped 


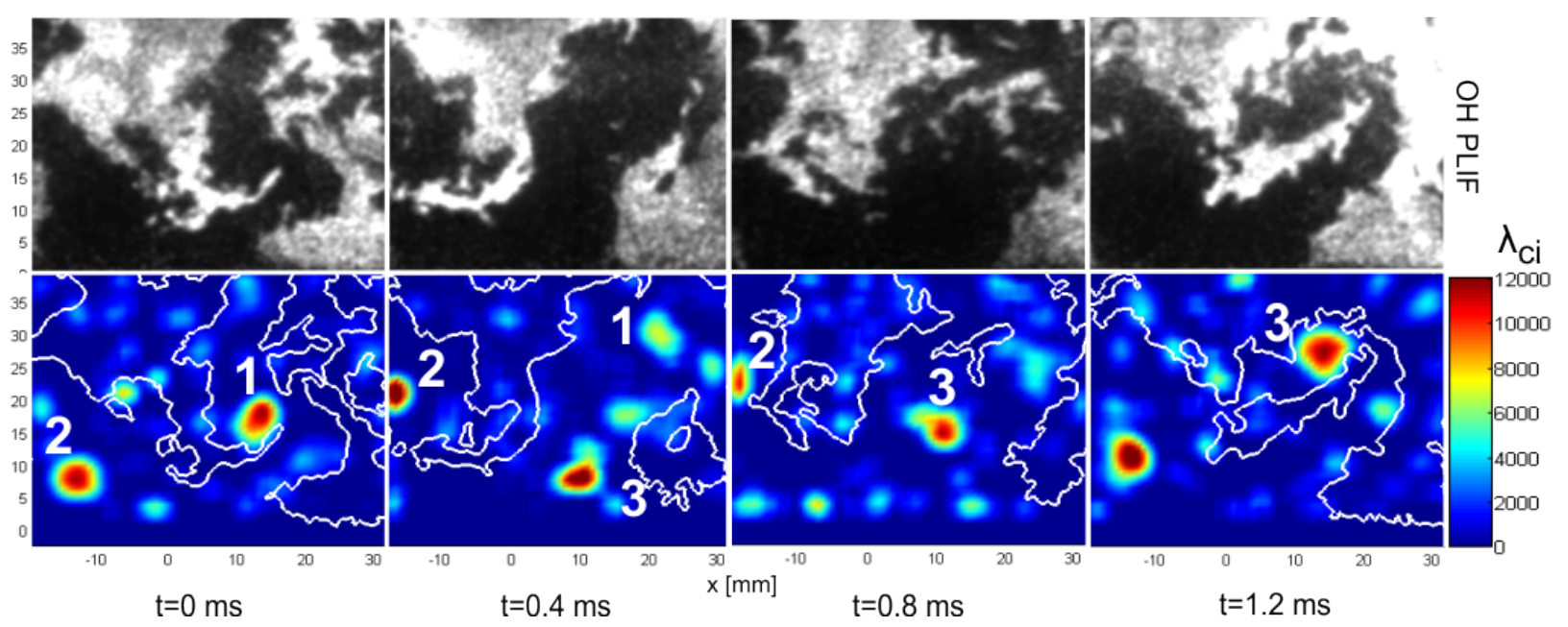

Figure 2.5: Time sequence showing the interactions between the flame and the helical vortex core. The flame is approximated by an iso-contour of the OH PLIF signal, shown on top, while the HVC is visualized through the swirling strength field. The vortices corrugate the flame locally, creating increased heat release.

around vortex 2. At this time, another part of the vortex crossed the measurement plane (numbered 3), though it was not yet strong or close enough to the flame to significantly affect it. As the HVC precessed, vortex 3 gained influence on the flame front, eventually bringing it back to its central position at $t=0.8 \mathrm{~ms}$ before wrapping up the flame at $t=1.2 \mathrm{~ms}$. From this description, it is clear that the flow and flame dynamics are highly dependent on the configuration of the HVC's intersection with the measurement plane, which is dependent of the HVC's azimuthal position. Further investigation into the importance of the HVC in flow-flame interactions requires more accurate prediction of the flame front's location and accurate quantification of the HVC's behaviour, which will be treated in the next chapter. 


\section{Chapter 3}

\section{Analytical Techniques}

The previous section presented a qualitative description of flow-flame behaviour in the swirl stabilized GTMC. The objective of this thesis requires that such flow-flame interactions be related to thermo-acoustic energy transfer, which in turn requires that acoustic pressure and heat release be quantified. Furthermore, important characteristics of the HVC, such as the precession frequency, have yet to be explored. This chapter will present the analytical techniques used to accurately quantify the pressure oscillations, local heat release rate, and $\mathrm{HVC}$ dynamics.

\subsection{Acoustics}

The acoustics of the chamber were measured using two microphone probes installed at distances of $15 \mathrm{~mm}$ and $55 \mathrm{~mm}$ from the nozzle exit plane. A microphone was also installed in the plenum of the burner. As seen in Fig. 3.1, the pressure signal of the two combustor probes were found to be in phase, indicating that the pressure field within the model combustor was spatially uniform. Also seen in Fig. 3.1 is the pressure signal from the plenum, which had a shape similar to that of the combustion chamber's, though the plenum and chamber signals were out of phase. Using cross correlation, the plenum pressure was found to lag the chamber pressure by roughly 60 to $80^{\circ}$, depending on the particular case and it's associated thermo-acoustic frequency. This frequency was obtained by Fourier transformation of the microphone signals, which showed a distinct 


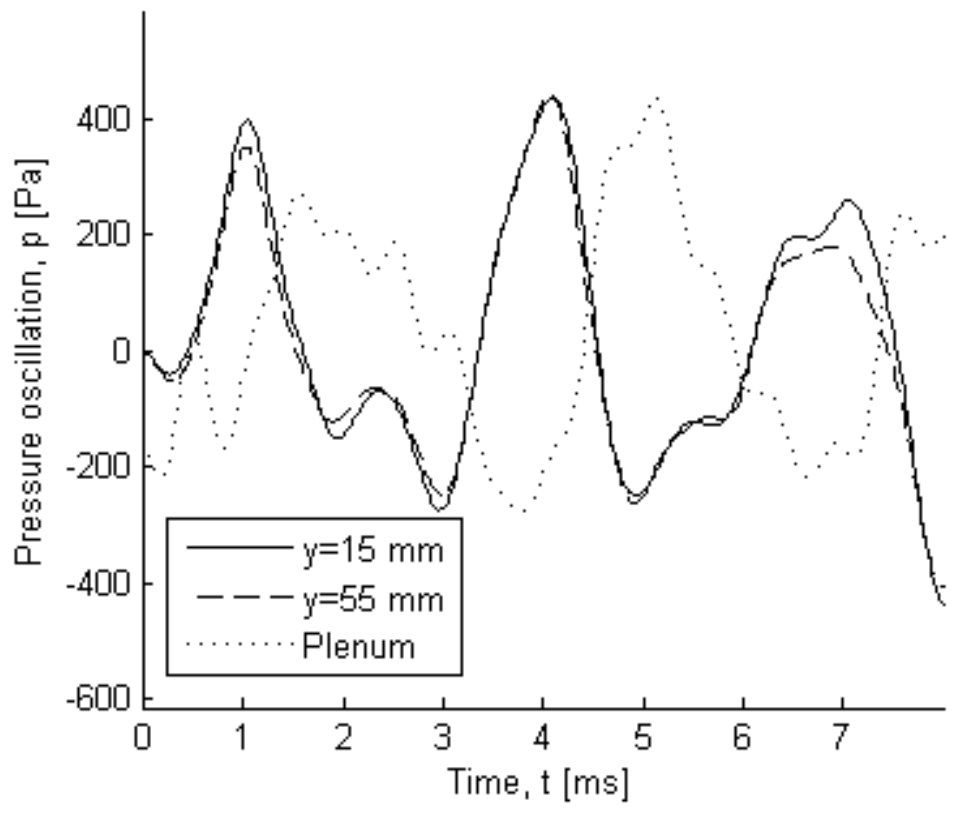

Figure 3.1: Pressure signal for case 6, taken at two different axial locations in the combustor and one location in the plenum.

peak in every experimental case. These peaks are shown in Fig. 3.2 for cases 1, 5b, and 6 , and the dominant frequency of the nine cases are listed along with the Root-MeanSquare (RMS) pressure oscillations $\left(p^{\prime \prime}\right)$ in Table 3.1. The plenum microphone signals exhibited less noise than those of the chamber microphones, and therefore they were used for all further analyses. To correct for the phase shift between the plenum $\left(p_{\mathrm{pl}}\right)$ and chamber $\left(p_{\mathrm{ch}}\right)$ signals, the plenum measurements were shifted by a value $(\Delta t)$ such that they overlapped the chamber signal in terms of phase. The value of $\Delta t$ was obtained by performing cross-correlation of the plenum and chamber measurements. That is, $\Delta t$ was chosen as the time at which the cross correlation reached its maximum value.

\subsection{HVC Dynamics}

To quantify the dynamics of the helical vortex core, swirling strength measurements were decomposed using proper orthogonal decomposition (POD), a statistical method of data analysis used to identify the most energetic modes of a system. POD decomposes a measurement $\Phi$ into a linear combination of mode shapes $M_{\mathrm{i}}(\mathbf{x})$, with each mode shape's contribution being a function of time via the temporal coefficient $a_{\mathrm{i}}(t)$. Mathematically, 


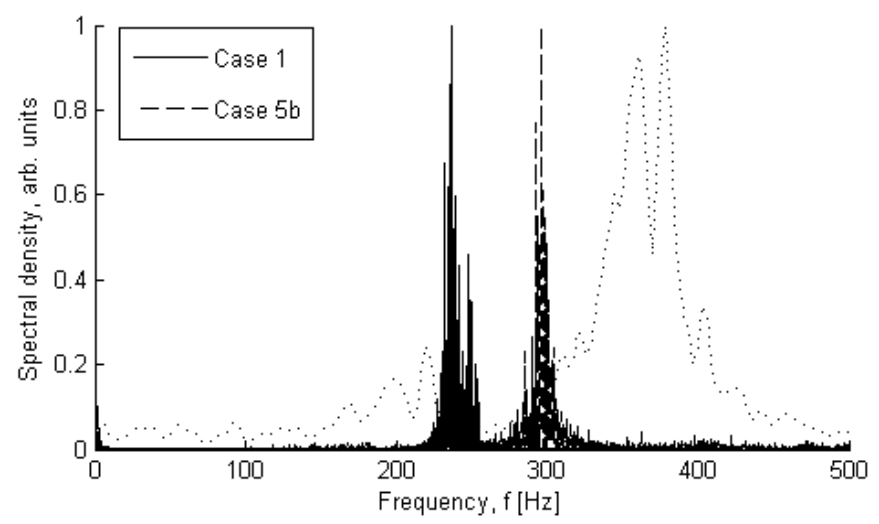

Figure 3.2: Acoustic frequency spectra of three different cases.

this is written

$$
\Phi_{k}(\mathbf{x}, t)=\sum_{i=1}^{k} a_{i}(t) M_{i}(\mathbf{x}),
$$

where $k$ is an arbitrary integer between 1 and $n$, the number of measurements. This is a generalisation of Singular Value Decomposition (SVD), a statistical method that performs the same decomposition with the number of modes, $m$, fixed by the matrix rank. The SVD theorem states that any $n$ measurement of rank $m$ may be factored in the form

$$
\Phi_{m n}=U_{m m} \Lambda_{m n} V_{n n}^{H}
$$

where $V^{\mathrm{H}}$ is the complex conjugate transpose (Hermitian) of $V$. The columns of $U$ are eigenvectors of $\Phi \Phi^{\mathrm{H}}, \Lambda$ is a diagonal matrix composed of the square roots of the eigenvalues of $U$ or $V$, and the columns of $V$ are eigenvectors of $\Phi^{\mathrm{H}} \Phi$. The proof of this theorem is beyond the scope of this thesis and the interested reader is referred to Ref. [100] where a detailed derivation of the proof can be found. Physically, the mode shapes are contained in the rows of matrix $U$ and the columns of $V$ represent the temporal coefficients, such that Eq. 3.2 is simply the matrix notation of Eq. 3.1 for $k=m$. The eigenvalues contained on the diagonal of $\Lambda$ represent the energy contribution of the corresponding modes. Typically, all three matrices are reordered such that the eigenvalues in $\Lambda$ are in decreasing order. This means that the first mode shape contained in $U$ will be the most energetic, followed by the second, third, and so on. One particularity of POD is that it provides optimal convergence of the kinetic energy, therefore identifying the most energetic orthogonal basis of a system. When performing POD, it is common practice to center data around the mean, such that the first mode represents a turbulent fluctuation. In this work, the mean flow pattern's intensity fluctuated in time as a result of thermo- 
acoustic coupling, and therefore POD was performed on the full data. As a result, the first mode $\left(M_{0}(x, y)\right)$ represented the long-time-averaged swirling strength field.

In order to pass from SVD to POD, that is, in order to obtain a mode decomposition of order $k$, one must solve

$$
\min \left\{|Y-\hat{Y}|^{2} \quad: \operatorname{rank}(\hat{Y})=k\right\}
$$

where $Y$ is the singular value decomposition of $\Phi(Y=U \Lambda V)$ and $\hat{Y}$ is the proper orthogonal decomposition of $\Phi$. Equation 3.3 is a least squares optimization problem and can readily be solved.

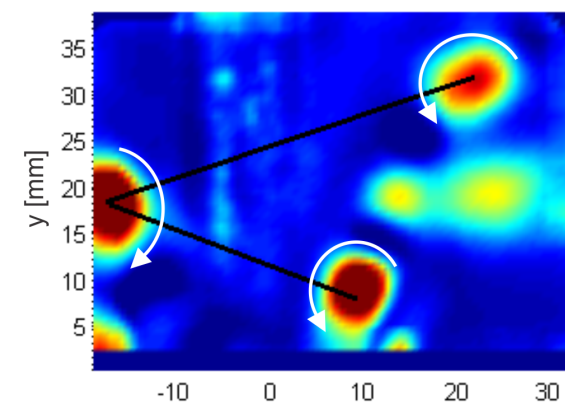

(a) $\mathrm{t}=0 \mathrm{~ms}$.

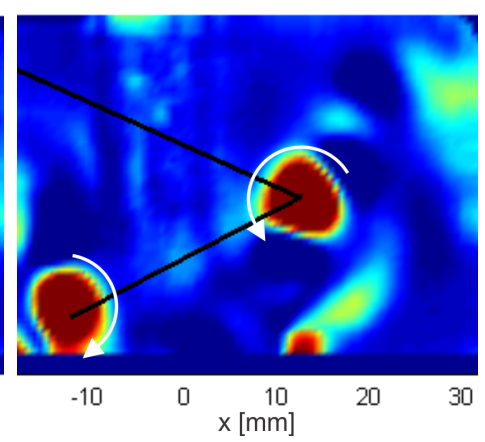

(b) $\mathrm{t}=0.7 \mathrm{~ms}$.

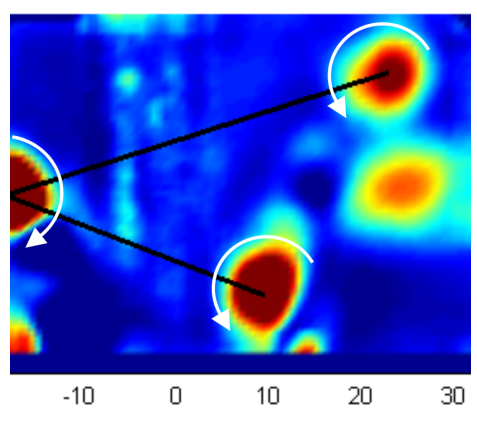

(c) $\mathrm{t}=1.3 \mathrm{~ms}$.

Figure 3.3: Instantaneous sequence showing the swirling strength reconstructed from the 10 first POD modes for the loudest. The staggered arrangement of the counter-rotating vortices is characteristic of the helical vortex core.

Figure 3.3 shows instantaneous swirling strength fields reconstructed from the first 11 (0 to 10) POD modes, which contained over $60 \%$ of the total kinetic energy of the flow. Steinberg et al. [101] demonstrated that the temporal coefficient associated with the first mode of the swirling strength POD $\left(a_{1}(t)\right)$ oscillated roughly sinusoidally at a frequency corresponding to the motion of the HVC around the burner and represented the HVC's precession around the combustion chamber in every experimental case. The relationship between the value of $a_{1}$ and the azimuthal position of the HVC may be verified by comparing instantaneous images of swirling strength fields with the value of $a_{1}$. Figure 3.4 shows the evolution of $a_{1}$ over a short time span, with the dashed lines at $\mathrm{t}=0, \mathrm{t}=0.7$, and $\mathrm{t}=1.3 \mathrm{~ms}$ corresponding to the time sequence in Fig. 3.3 . From $\mathrm{t}=0$ to $\mathrm{t}=0.7 \mathrm{~ms}$, the temporal coefficient underwent half of an oscillation. During the same time period, the HVC underwent half of a rotation, as indicated by the anti-symmetry along the burner 


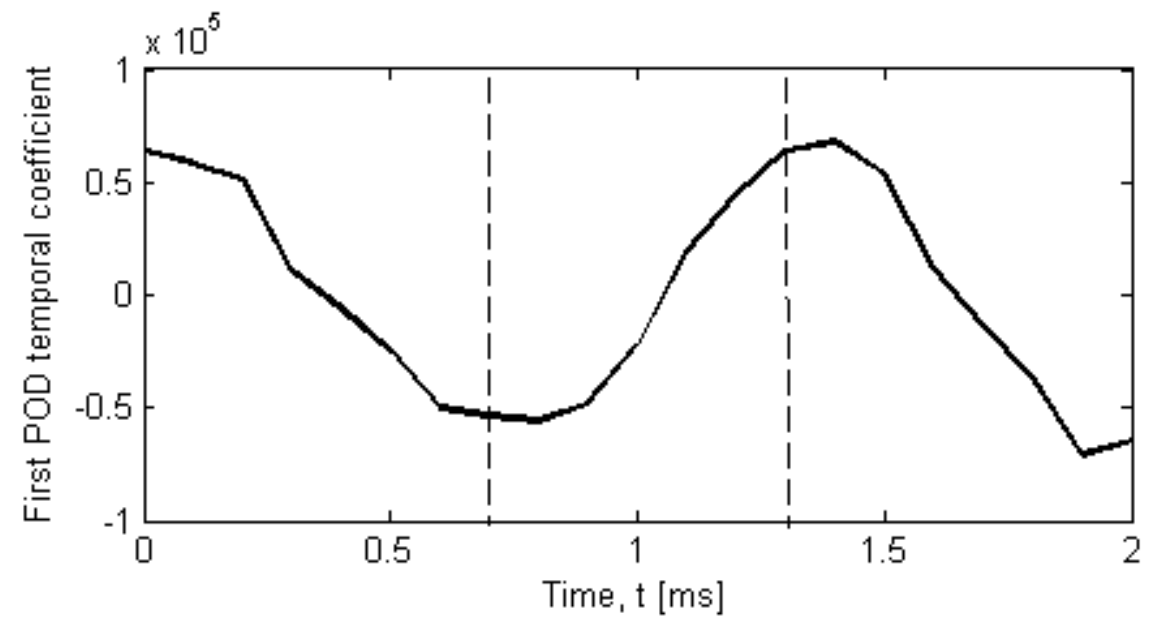

Figure 3.4: Evolution of $a_{1}(t)$ over the time span shown in Figure 3.3. Dashed lines are at $\mathrm{t}=0, \mathrm{t}=0.7$, and $\mathrm{t}=1.3 \mathrm{~ms}$, corresponding to the times in Figure 3.3.

axis between Figs. 3.3(a) and 3.3(b). Similarly, after $1.3 \mathrm{~ms}$, the temporal coefficient completed an entire cycle and the HVC rotated $360^{\circ}$ in the burner. Therefore, the phase of the temporal coefficient can be taken as a measure of the azimuthal position of the HVC from an arbitrary starting point. It follows that the frequency at which $a_{1}$ oscillates is a measure of the rotation frequency of the HVC. The frequency spectra of $a_{1}$ were computed by FFT and contained a distinct peak, shown for representative quiet, intermediate, and loud cases $\left(3,5 \mathrm{~b}\right.$, and 6 , respectively) in Fig. 3.5. The frequency $\left(f_{\mathrm{h}}\right)$ corresponding to the peak in the $a_{1}$ spectrum is shown in Table 3.1 for each case containing an HVC. As can be seen, the HVC's frequency was different than the thermoacoustic frequency in every case.

\subsection{Doubly Phase Resolved Analysis}

The qualitative analysis of flow-flame interactions of Sec. 2.2.2, along with past research [73, 101], have shown that both the azimuthal position of the HVC and the acoustic phase had an important influence on the rate of heat release. Therefore, understanding the coupled flame and flow dynamics required compilation of statistics as a function of both acoustic $\left(\phi_{\mathrm{a}}\right)$ and azimuthal position around the $\operatorname{HVC}\left(\phi_{\mathrm{h}}\right)$ [73, 101], which was derived from the POD temporal coefficient associated to the most energetic turbulent mode. That is, any quantity $\zeta$ was decomposed into the linear combination of a long time 


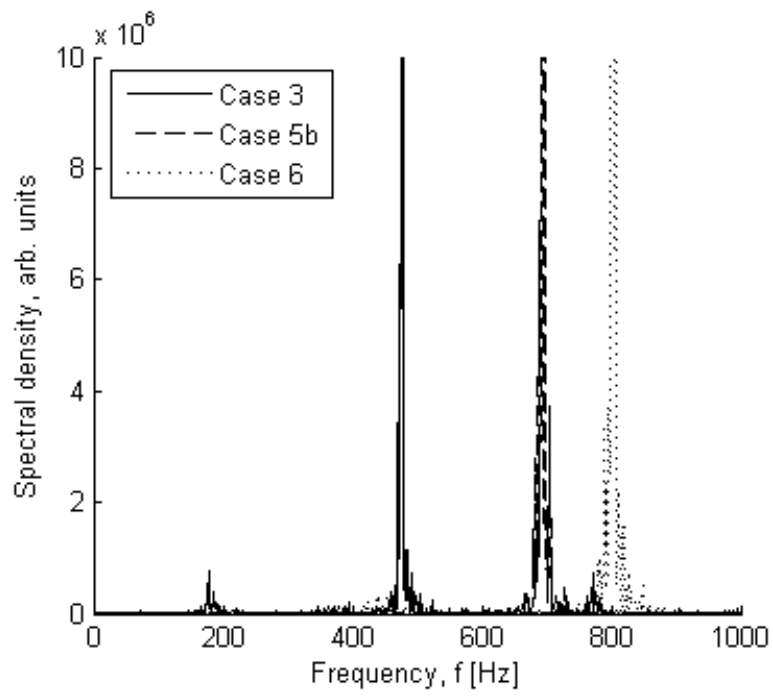

Figure 3.5: Frequency spectra of $a_{1}(t)$. The peak indicates the rotation frequency of the HVC

\begin{tabular}{cccccc} 
Case & $\phi$ & $P_{\mathrm{th}}[k W]$ & $p \prime \prime[\mathrm{Pa}]$ & $f_{\mathrm{ac}}[\mathrm{Hz}]$ & $f_{\mathrm{v}}[\mathrm{Hz}]$ \\
\hline & & & & & \\
\hline 1 & 0.7 & 10 & 19.4 & 237 & 234 (Toroidal) \\
2 & 0.7 & 15 & 20.2 & 270 & 300 (Toroidal) \\
3 & 0.7 & 20 & 107.4 & 296 & 475 \\
4 & 0.7 & 25 & 74.4 & 306 & 578 \\
\hline $5 \mathrm{a}$ & 0.65 & 30 & 145.4 & 289 & 749 \\
$5 \mathrm{~b}$ & 0.7 & 30 & 149.1 & 314 & 693 \\
$5 \mathrm{c}$ & 0.75 & 30 & 177.2 & 418 & $\mathrm{~N} / \mathrm{A}$ \\
$5 \mathrm{~d}$ & 0.80 & 30 & 105.2 & 430 & $\mathrm{~N} / \mathrm{A}$ (Toroidal) \\
\hline 6 & 0.7 & 35 & 181.9 & 380 & 799
\end{tabular}

Table 3.1: List of the different cases of the experiment and corresponding equivalence ratio $\phi$, themal power $P_{\mathrm{th}}$, RMS acoustic amplitude $p /$, dominant acoustic frequency $f_{\mathrm{ac}}$, and dominant vortical frequency $f_{v}$. Unless otherwise specified, the dominant vortical structure is the helical vortex core. Vortex frequencies that were unavailable due to bad data, or the lack of a dominant mode, are denoted N/A.

average component $\bar{\zeta}$, a doubly phase resolved component $\zeta^{\mathrm{ah}}\left(\phi_{\mathrm{a}}, \phi_{\mathrm{h}}\right)$, and a turbulent fluctuation $\zeta^{\mathrm{t}}(t)$, such that

$$
\zeta(\mathbf{x}, t)=\bar{\zeta}(\mathbf{x})+\zeta^{\mathrm{ah}}\left(\mathbf{x}, \phi_{\mathrm{a}}, \phi_{\mathrm{h}}\right)+\zeta^{\mathrm{t}}(\mathbf{x}, t)
$$


The decomposition is shown schematically in Fig. 3.6 for a single oscillatory process. Such statistics are useful in determining the effect of either the HVC or the thermoacoustic oscillations on a quantity. Furthermore, in the scope of this project, they are necessary for the calculation of thermo-acoustic energy transfer, as will be discussed in Sec. 4.1.

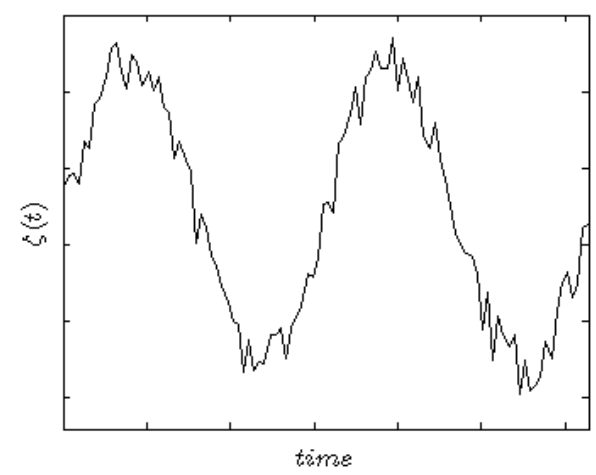

(a) Measurement.

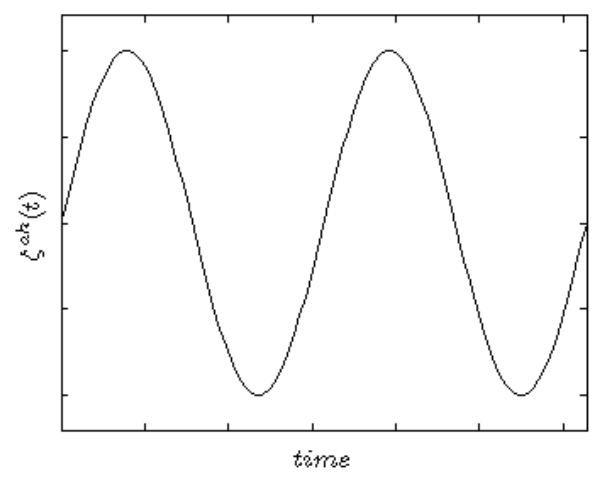

(c) Phase component.

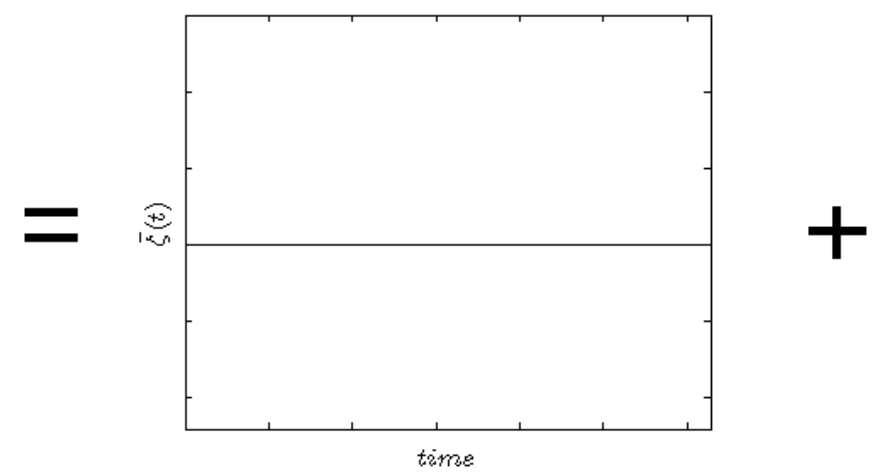

(b) Long time average component.

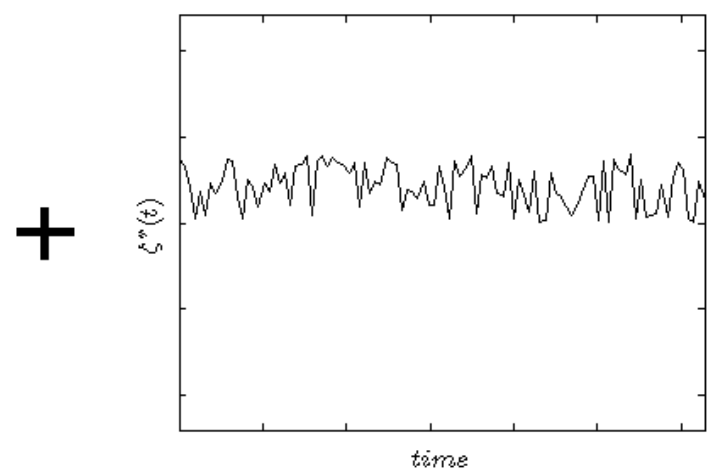

(d) Random component.

Figure 3.6: Measurement decomposed into a long time average, phase, and random component.

Doubly phase resolved statistics of experimental results were obtained by dividing both the acoustic signal and the POD temporal coefficient of the first mode into sections through the introduction of phase numbers, as shown in Fig. 3.7. Throughout the rest of this thesis, all phase angles will be given in terms this numbering scheme. A measurement taken at any given time was associated to the nearest acoustic and HVC 
phase number combination, and all measurements with a common combination of phase number were averaged. This is illustrated in Fig. 3.8. Mathematically, the averaging operation eliminates the random fluctuation term in Eq. 3.4, resulting in a doubly-phaseaveraged component, $\bar{\zeta}^{\text {ah }}$, defined as

$$
\bar{\zeta}^{\mathrm{ah}}\left(\mathbf{x}, \phi_{\mathrm{a}}, \phi_{\mathrm{h}}\right)=\bar{\zeta}(\mathbf{x})+\zeta^{\mathrm{ah}}\left(\phi_{\mathrm{a}}, \phi_{\mathrm{h}}\right)
$$

Using the 12 phase angles defined in Fig. 3.7, this implies a total of 144 different phase combinations. A typical measurement sequence consisted of roughly 8000 measurements, for an average of 50-60 measurements per phase combination. For better statistical convergence, two sequences were used for a given case, resulting in approximately 100-110 measurements per phase combination. Furthermore, since the POD temporal coefficient is a measure of the azimuthal position of the vortex from an arbitrary starting point (see Sec. 3.2), doubly phase resolved statistics can be used to visualize processes in three dimensions, albeit with low azimuthal resolution. This is achieved by treating the HVC phase as a physical azimuthal plane in a reference frame that rotates with the HVC. That is, at a particular acoustic phase, 12 different planar fields represent slices of the HVC at different azimuthal locations. When performing this reconstruction, only data measured on the right side of the burner's axis of symmetry was considered. This was to avoid a discontinuity in the 3-D reconstructions that would have resulted from the fact that measurements were not radially centred about the burner's axis. This would have created an overlap between statistics at opposing azimuthal positions around the HVC. For this reason, and because the measurement plane spanned a smaller distance to the left of the burner's axis of symmetry than to the right, all doubly phase resolved statistics will be presented on the right hand side of the burner's axis of symmetry only.

\section{Notation Relevant to Doubly Phase Resolved Analysis}

Many of the operations that can be performed on instantaneous data can also be performed on the doubly phase resolved statistics. Spatial averaging, integrals, and computation of oscillations are three operations that will be required in the subsequent analysis, for which distinct notation is laid out here to differentiate them from the equivalent operations on instantaneous data. 


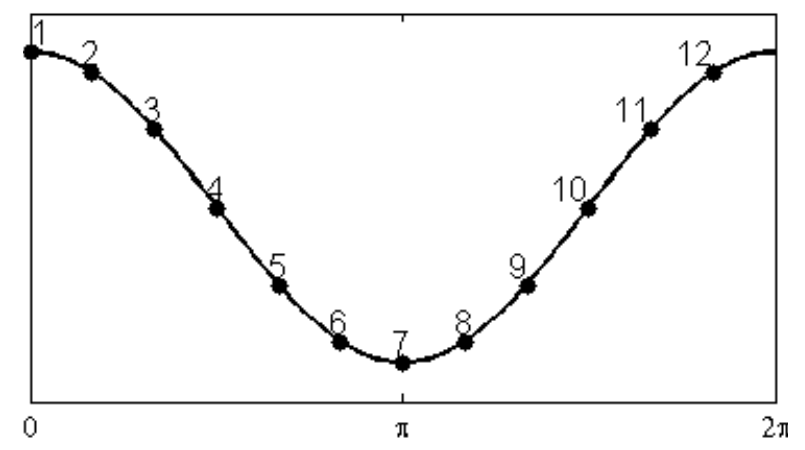

Figure 3.7: Definition of phase numbers used for doubly phase resolved analysis throughout the research project.

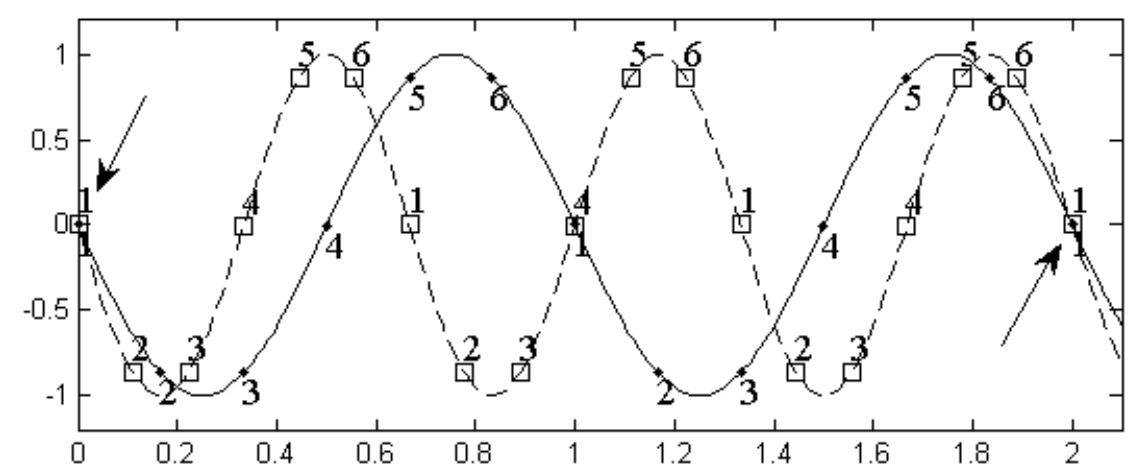

Figure 3.8: Schematic of two sinusoidal signals, separated into 6 phase numbers for clarity. The different frequencies of the two signals make for repeating phase combinations at times 0 and 2, respectively (pointed out by arrows). These measurements may be therefore averaged to obtain a doubly phase resolved statistic.

To study the behaviour of an arbitrary variable $\zeta$ as a function of the combustor's axial position, it's useful to calculate the mean value of this variable over the combustor's cross-section, denoted $\langle\zeta\rangle$, and defined as

$$
\langle\zeta\rangle\left(y, \phi_{\mathrm{a}}\right)=\int_{\phi_{\mathrm{h}}} \int_{x} \bar{\zeta}^{\mathrm{ah}}\left(x, y, \phi_{\mathrm{h}}, \phi_{\mathrm{a}}\right) \mathrm{d} x \mathrm{~d} \phi_{\mathrm{h}},
$$

where the denominator and the multiplication of $\bar{\zeta}^{\text {ah }}$ by $x_{\mathrm{i}}$ are included to compensate for the cylindrical nature of the co-ordinate system associated to the doubly phase resolved data (since $\phi_{\mathrm{h}}$ is a measure of the azimuthal position around the HVC).

The spatially integrated value of a variable at a given HVC phase was also a quantity of interest. This was obtained by integrating the variable over the $x$ and $y$ coordinates 


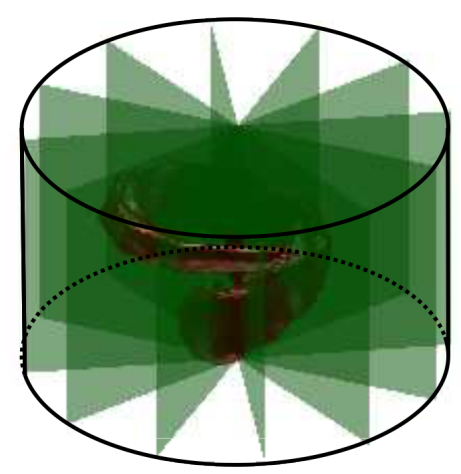

(a) Domain of doubly phase resolved statistics.

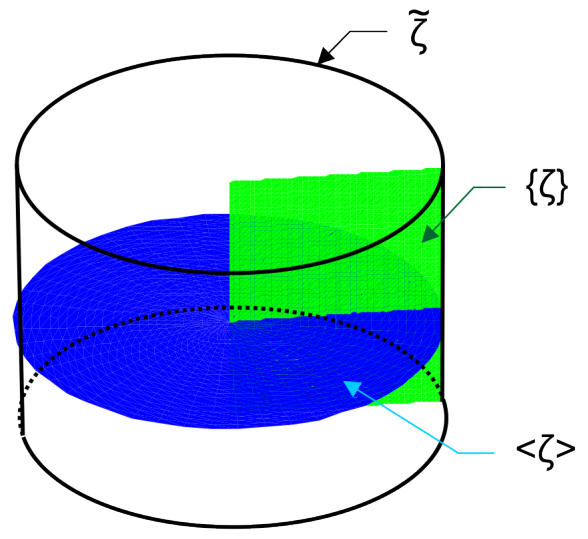

(b) Cylindrical domain with cross-section (blue) and doubly phase resolved data plane (green), representative of the domains over which important statistics are computed.

Figure 3.9: Domain of doubly phase resolved statistics around a helical vortex.

at a given HVC phase:

$$
\{\zeta\}\left(\phi_{\mathrm{a}}, \phi_{\mathrm{h}}\right)=\int_{x} \int_{y} \bar{\zeta}^{\mathrm{ah}}\left(x, y, \phi_{\mathrm{a}}, \phi_{\mathrm{h}}\right) \mathrm{d} y \mathrm{~d} x .
$$

The result could then be integrated over the HVC phases to obtain an approximation to the integral over a cylindrical domain:

$$
\begin{aligned}
\tilde{\zeta}\left(\phi_{\mathrm{a}}\right) & =\int_{\phi_{\mathrm{h}}}\{\zeta\}\left(\phi_{\mathrm{a}}, \phi_{\mathrm{h}}\right) \mathrm{d} \phi_{\mathrm{h}} \\
& =\int_{\phi_{\mathrm{h}}} \int_{x} \int_{y} \bar{\zeta}^{\mathrm{ah}}\left(x, y, \phi_{\mathrm{a}}, \phi_{\mathrm{h}}\right) \mathrm{d} y \mathrm{~d} x \mathrm{~d} \phi_{\mathrm{h}} .
\end{aligned}
$$

To better understand the physical significance of these three computations, it is helpful to represent the different domains of computation schematically. The doubly phase resolved domain is represented by green rectangular surfaces in Fig. 3.9(a), with each rectangular plane associated to a different HVC phase. An HVC is shown in red for spatial referencing. This domain evolves in a 3-D space in the shape of a circular cylinder, shown in black. $\langle\zeta\rangle\left(y, \phi_{\mathrm{a}}\right)$ represents averaging of a measurement over a planar crosssection of this cylinder, shown in blue in Fig. 3.9(b), while $\{\zeta\}$ is obtained by integrating the data at a given HVC phase, represented by the green rectangular plane in Fig. 3.9(b). For an approximation to the value of the integrated variable over the whole cylindrical domain $(\tilde{\zeta})$, the measurement may subsequently be integrated with respects to $\phi_{\mathrm{h}}$.

Another important quantity for the analysis of thermo-acoustic velocity coupling is the oscillation over the acoustic cycle. Spatially resolved oscillations over the acoustic 


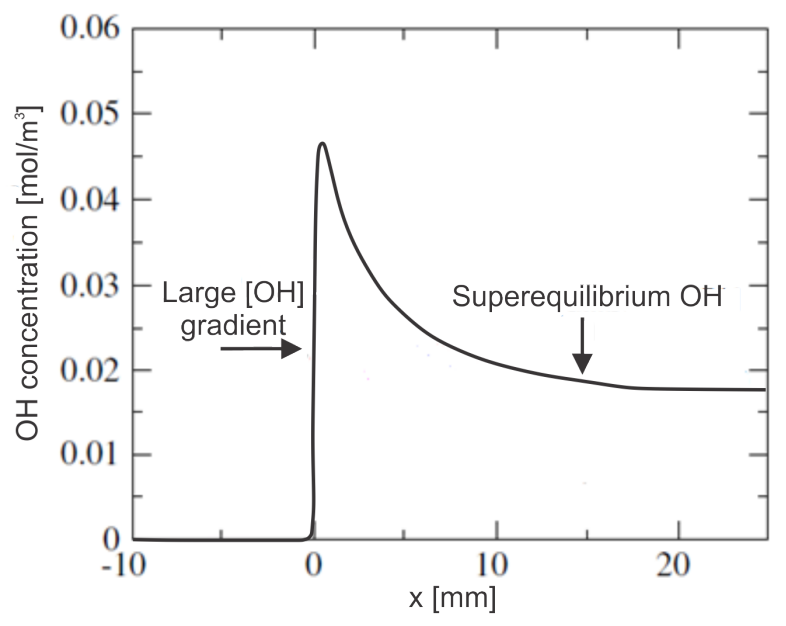

Figure 3.10: Typical OH distribution across a stoichiometric laminar flame front. Note the high gradient near the flame front $(\mathrm{x}=0)$ and the superequilibrium $\mathrm{OH}$ on the product side of the flame $(\mathrm{x}>0)$.

cycle can be computed by taking the difference between the local doubly phase resolved average and the local average over the acoustic cycle:

$$
\zeta^{\mathrm{a}}\left(x, y, \phi_{\mathrm{a}}, \phi_{\mathrm{h}}\right)=\bar{\zeta}^{\mathrm{ah}}\left(x, y, \phi_{\mathrm{a}}, \phi_{\mathrm{h}}\right)-\frac{1}{n_{\phi_{\mathrm{a}}}} \sum_{\phi_{\mathrm{a}}=1}^{n_{\phi_{\mathrm{a}}}} \bar{\zeta}^{\mathrm{ah}}\left(x, y, \phi_{\mathrm{a}}, \phi_{\mathrm{h}}\right),
$$

where $n_{\phi_{\mathrm{a}}}=12$ is the number of acoustic phases used in the analysis. Note that for the remainder of this thesis, doubly phase resolved oscillations over the acoustic cycle will be referred to as fluctuations. Also, the superscipt $a$ notation can be applied to any further manipulation of the variable $\zeta$. For example, $\tilde{\zeta}^{\text {a }}$ represents the fluctuation of the spatially integrated variable over the volume of the doubly phase resolved cylindrical domain.

\subsection{Flame Finding}

As a first step towards quantifying spatially resolved heat release, this section will discuss how the flame fronts may be identified from the laser diagnostics measurements. These flame fronts will then be used in combination with doubly phase resolved statistics to approximate the spatially-resolved heat release rate. Kortschik et al. [102] proposed a method of identifying flame fronts by making use of Rayleigh scattering measurements, the latter being used to map the temperature of the flow field. Applying a threshold 
to the temperature field allowed them to create a binary image of the reaction zone, setting all regions where $\mathrm{T} \geq 800 \mathrm{~K}$ to one and $\mathrm{T}<800 \mathrm{~K}$ to zero. Although this method was successful, it couldn't be implemented in the current research because of the absence of Rayleigh scattering data and no other means of establishing the temperature field quantitatively. Hence, another method was implemented, as described by Steinberg et al. [101]. This method makes use solely of OH PLIF data and knowledge of the $\mathrm{OH}$ distribution across a flame front (shown in Fig. 3.10). Specifically, the algorithm relies on the high $\mathrm{OH}$ concentration gradient at the flame front, super-equilibrium $\mathrm{OH}$ on the product side, and low, positive $\mathrm{OH}$ curvature on the product side. The validity of this algorithm relies on the assumption of a flamelet regime.

The specifics steps in the flame finding algorithm are as follows:

1. Calculate the OH PLIF gradient.

2. Obtain the magnitude of the PLIF gradient (Shown in Fig. 3.11(b)).

3. Apply a threshold to the PLIF gradient of step 2, and binarise the gradient field so that any gradient value below the threshold becomes 0 , and any value above the threshold becomes 1 . The binary field, $\beta$, corresponding to Fig. 3.11(b) is shown in Fig. 3.11(c).

4. Take the $\beta=0.5$ isocontours.

5. Examine the OH PLIF value (Fig. 3.11(a)) at every point on the isocontours of step 4. If the value is above a certain threshold, this indicates that the point is in a region of supercritical OH PLIF, and should be discarded.

6. Examine the OH PLIF curvature value (Fig. 3.11(d)) at every remaining point on the isocontours of step 4. If the value is above a certain threshold, then that point is on the product's side and should be discarded.

Any point that has not been discarded by the end of step 6 is considered to be part of the flame front. 


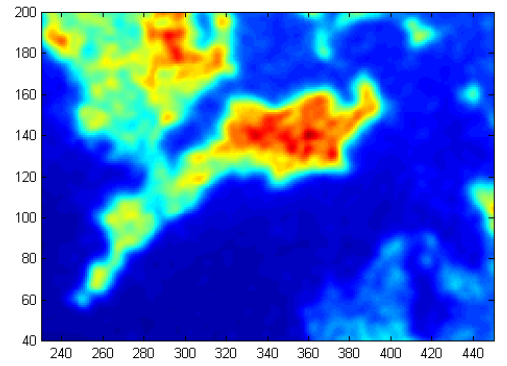

(a) $\mathrm{OH}$ distribution.

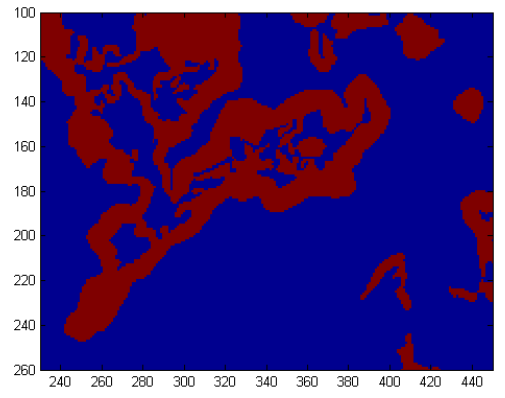

(c) Thresholded $\mathrm{OH}$ distribution gradient.

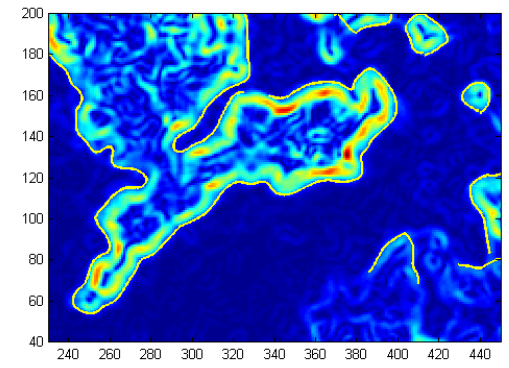

(b) $\mathrm{OH}$ gradient distribution and detected flame fronts.

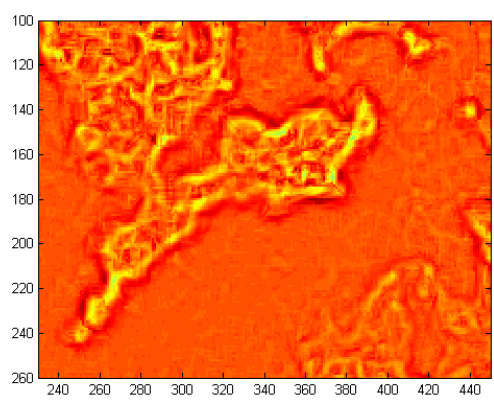

(d) $\mathrm{OH}$ curvature.

Figure 3.11: Important scalar fields in flame finding algorithm

\subsection{Flame Surface Density}

Recall that the objective of the thesis is to identify flow flame interactions leading to high and low thermo-acoustic energy transfer. This requires knowledge of spatially resolved volumetric heat release rate, which heretofore has not been quantified. The volumetric heat release is proportional to the volumetric rate of conversion from reactants to products $(\dot{\omega})$, for which the following relation applies [104]:

$$
\dot{\omega}=\rho_{\mathrm{u}} S_{\mathrm{L}}^{0} I_{0} \Sigma
$$

where $\rho_{\mathrm{u}}$ is the unburnt gas density, $S_{\mathrm{L}}^{0}$ is the unstretched laminar flame speed, $I_{0}$ is a correction factor for $S_{\mathrm{L}}^{0}$ arising from flame stretch, and $\Sigma$ is the flame surface density (FSD) [105]. The FSD is defined as the flame sheet surface per unit volume which, based on two dimensional measurements, reduces to flame surface length per unit area. In Eq. 


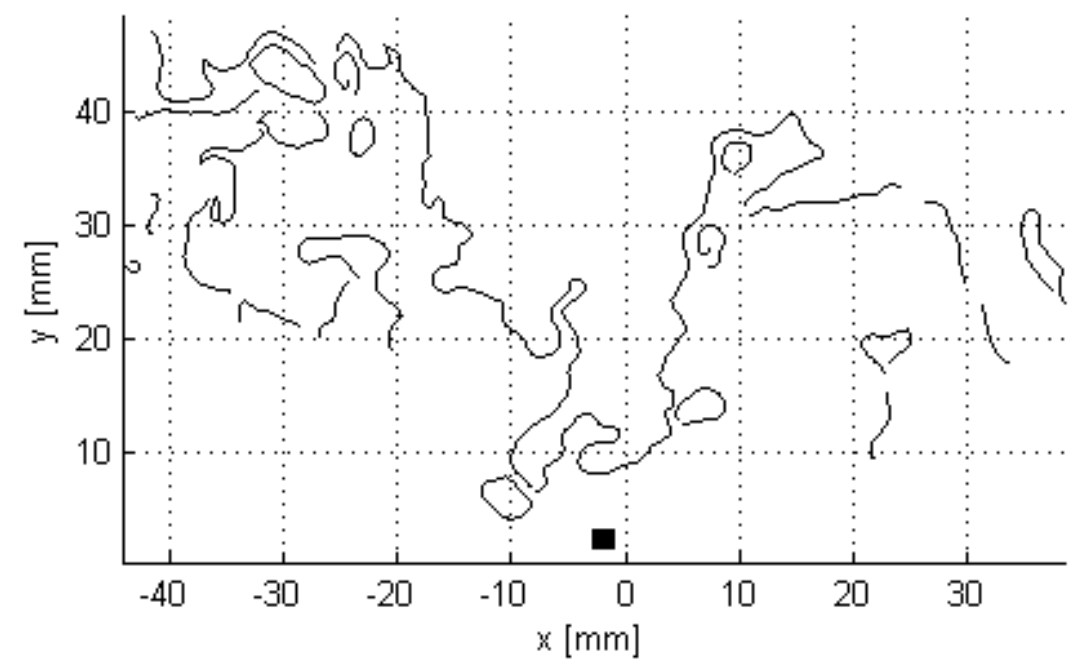

Figure 3.12: Hypothetical grid division of the PLIF measurement plane for calculation of the flame surface density. The actual grid division was 5x finer, represented as a black square.

$3.11 S_{\mathrm{L}}$ is determined by reactant composition, temperature and pressure [46], while for a methane-air flame in low to intermediate turbulence intensities, $I_{0}$ can be assumed equal to 1. The FSD may therefore be used to approximate the heat release rate distribution, an important factor in thermo-acoustic coupling as given by the Rayleigh integral.

Shepherd [105] has proposed a method of calculating the flame surface density based on a progress variable $\bar{c}$, which has a value of 0 in the reactants and 1 in the products. The flame length and flame zone area may then be expressed as a function of the progress variable and used to find the FSD. For this method to be employed, a reliable method for mapping degree of conversion, such as PLIF of the fuel, must be available. Since this is not the case for the current experiment, another method of calculating FSD was devised. First, the PLIF measurement plane was divided into a grid of known dimensions. This is illustrated in Fig. 3.12. The total flame sheet length of a given grid cell was found by computing the sum of the lengths of every instantaneous flame front within the cell. Once this quantity was known, the FSD of the given cell $\left(\Sigma_{\mathrm{i}}\right)$ was calculated by dividing 
the total flame sheet length $\left(l_{\mathrm{f}, \mathrm{i}}\right)$ by the grid cell's area $\left(A_{\mathrm{i}}\right)$ :

$$
\Sigma_{i}=\frac{l_{\mathrm{f}, \mathrm{i}}}{A_{i}}
$$

One consequence of the use of this method for calculating the FSD is that the resolution of the computed field will always be considerably lower than that of the PLIF field. In this thesis, the flames fronts are obtained with the same resolution as the $\mathrm{OH}$ PLIF images, using the algorithm described in the last section. The resolution of the FSD fields are five times lower than those of the PLIF fields, meaning that each FSD grid cell consists of 10x10 PLIF image pixels (approximately $1.7 \times 1.7 \mathrm{~mm}$ ). 


\section{Chapter 4}

\section{Results}

\subsection{Thermo-Acoustic Energy Transfer}

Calculation of the thermo-acoustic energy transfer requires evaluation of the Rayleigh integral (Eq. 1.10, repeated here for convenience).

$$
\Theta=\frac{\gamma-1}{\gamma} \frac{1}{\bar{p}} \int_{V} \int_{t} p^{\prime}(\mathbf{x}, t) \dot{q}^{\prime}(\mathbf{x}, t) \mathrm{d} t \mathrm{~d} \mathbf{x}
$$

As described in the previous chapter, spatio-temporally resolved heat release rate measurements $\left(\dot{\mathrm{q}}^{\prime}(\mathbf{x}, \mathrm{t})\right)$ were not available. Given the combination of fuel and combustion regime of the experiments, the heat release rate was approximately proportional to the flame surface area. Hence, flame surface density is a good approximation for the volumetric heat release rate distribution. Since the FSD is a statistical quantity, a statistical analogue to the Rayleigh integral was required. To this end, the temporal integral of Eq. 1.10 was replaced with an integral over the acoustic period, allowing for the doubly phase resolved statistics to be used. The resulting expression is

$$
\Psi \propto \int_{\mathbf{x}} \int_{\phi_{\mathrm{h}}} \int_{\phi_{\mathrm{a}}} p^{\mathrm{a}}\left(\phi_{\mathrm{a}}\right) \Sigma^{\mathrm{a}}\left(\mathbf{x}, \phi_{\mathrm{h}}, \phi_{\mathrm{a}}\right) \mathrm{d} \phi_{\mathrm{a}} \mathrm{d} \phi_{\mathrm{h}} \mathrm{d} \mathbf{x},
$$

where $p^{\mathrm{a}}$ and $\Sigma^{\mathrm{a}}$ are the doubly-phase-resolved pressure and flame surface density fluctuations respectively.

The relationship between the overall thermo-acoustic energy transfer computed using Eq. 4.1 and the measured pressure oscillation amplitude is shown in Fig. 4.1, clearly 


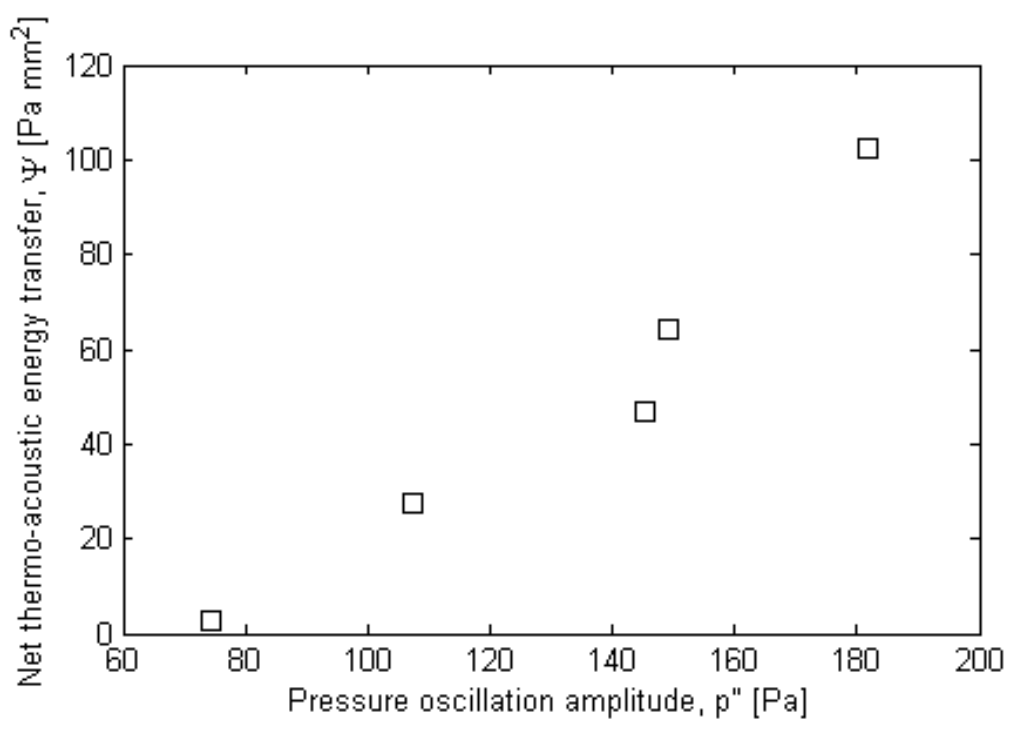

Figure 4.1: Thermo-acoustic energy transfer as a function of pressure oscillation.

demonstrating that $\Psi$ is a good representation of the thermo-acoustic coupling. However, $\Psi$ provides no insight into the underlying processes responsible for the thermo-acoustic energy transfer. To gain insight into these processes, the distribution of the volumetric thermo-acoustic energy transfer, $\psi$, defined by

$$
\psi\left(\mathbf{x}, \phi_{\mathrm{h}}\right) \propto \int_{\phi_{\mathrm{a}}} p^{\mathrm{a}}\left(\phi_{\mathrm{a}}\right) \Sigma^{\mathrm{a}}\left(\mathbf{x}, \phi_{\mathrm{a}}, \phi_{\mathrm{h}}\right) \mathrm{d} \phi_{\mathrm{a}},
$$

was analysed. Figures $4.2(\mathrm{a})$ to $4.2(\mathrm{e})$ show $\psi$ for all the experimental cases with HVCs. Regions of high positive and negative $\psi$ are shown by green and blue isosurfaces respectively, while the helical vortex is displayed with a red vorticity isosurface. From these figures, it may be noted that each case exhibits similar $\psi$ distributions. Notably, each case has two distinct regions of coupling:

1. An inner coupling region (ICR) composed of positive and negative $\psi$ zones, denoted by 1 and 2 respectively, that are bound by the helix.

2. An outer coupling region (OCR), denoted by 3, that surrounds the helix.

For the quieter cases, the ICR contained mostly positive $\psi$, whereas the OCR contained mostly negative $\psi$. For the louder cases, the net $\psi$ in the ICR became approximately zero, whereas the OCR changed from negative to positive $\psi$. Hence, the OCR is responsible for most of the positive thermo acoustic coupling in the louder flames. Furthermore, at a given azimuthal position, $\psi$ in the ICR and OCR had opposite signs. 


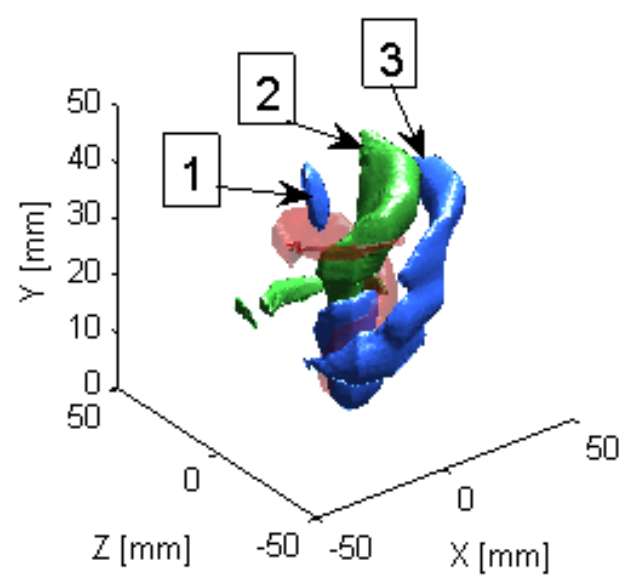

(a) Case 3 .

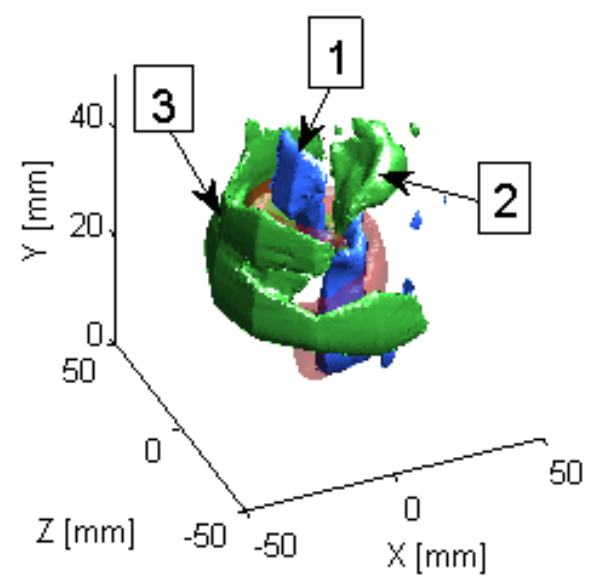

(c) Case 5b.

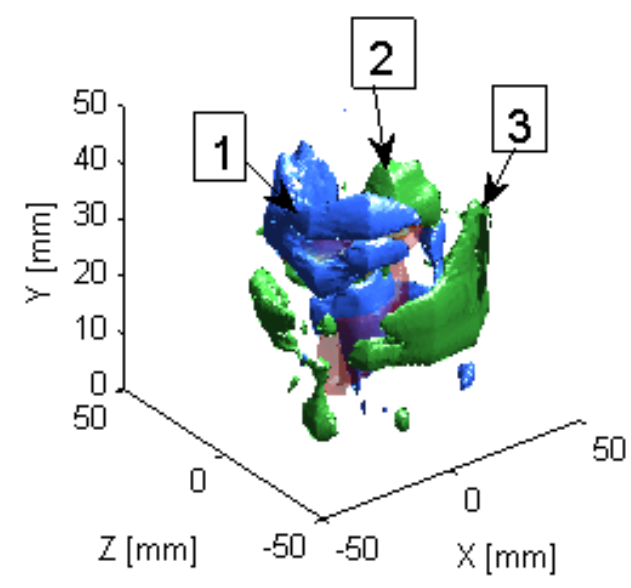

(b) Case 4 .

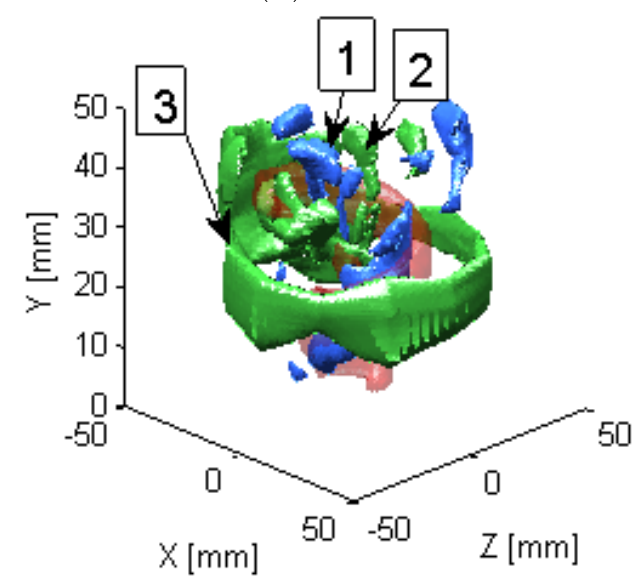

(d) Case 5a.

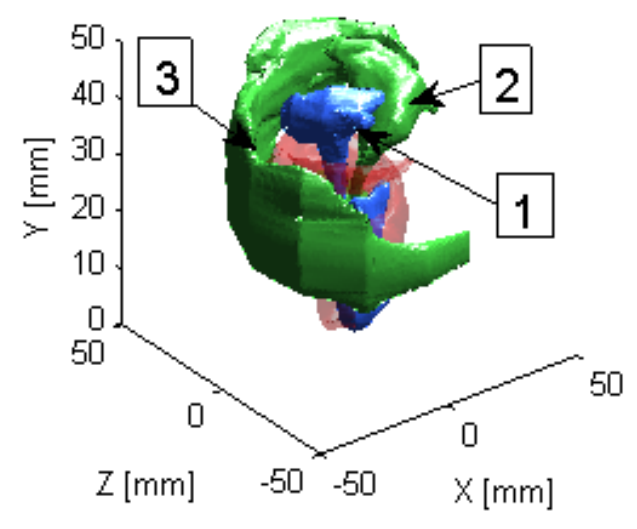

(e) Case 6 .

Figure 4.2: Isorsurfaces of volumetric thermo-acoustic energy transfer $(\psi)$ of the studied cases, in order of increasing thermal power $\left(\mathrm{P}_{\mathrm{th}}\right)$. The red isosurface is vorticity at 5000 $\mathrm{s}^{-1}$. Numbers 1 and 2 point to regions of negative and positive $\psi$ in the ICR, respectively. Number 3 points to the OCR. As the thermal power increases, the net thermo-acoustic energy transfer increases in the OCR, and approaches 0 in the ICR. 


\subsection{Flow-Flame Interactions Causing $\psi$ Distributions}

With an essentially spatially uniform pressure field in the combustion chamber, the modified Rayleigh integral shows that the spatial distribution of $\psi$ is set by the spatial and phase (relative to the pressure) distribution of $\Sigma^{\mathrm{a}}$. This distribution can be influenced by a number of flow- and flame-related factors, including variations in flame corrugations, periodic local flame extinctions, and large-scale flame motions associated with bulk flow oscillations.

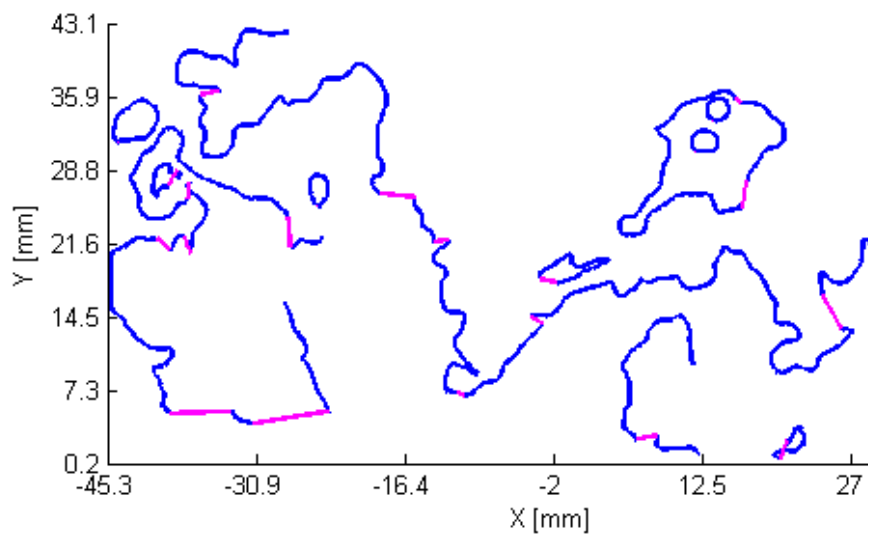

(a) Instantaneous local flame extinctions.

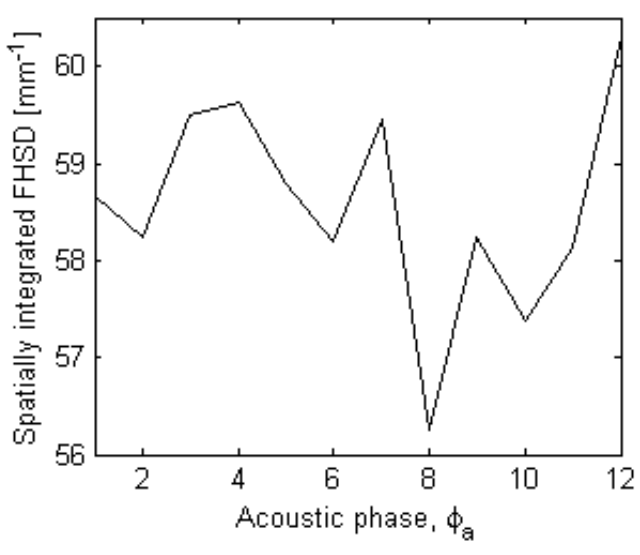

(b) Net FHSD for case 6.

Figure 4.3: Flame holes (a) used to compute the net flame hole surface density (b). Flames are represented as blue lines, while the flame holes are shown in magenta. Note that the net FHSD is not periodic over the acoustic cycle.

Periodic local flame extinctions were quantified by computing the flame hole surface density (FHSD). Using the flame fronts obtained with the algorithm of Sec. 3.4, local flame extinctions were approximated by tracing a line between the extremities of two flame fronts. Since a local extinction necessarily produces two extremities by splitting a flame front, a condition was imposed to limit the number of links of a given flame front extremity to one. Figure 4.3(a) shows the flame fronts (blue) and corresponding local extinctions (magenta) of an instantaneous flame image for case 6. These approximate flame extinctions were expressed statistically by the FHSD, computed using the algorithm of Sec. 3.5, and were found to fluctuate randomly over the acoustic cycle, as seen in Fig. 4.3(b). Furthermore, if local flame extinctions are assumed to be the principle mechanism causing fluctuations in heat release, an increase in FHSD should be accompanied by a 


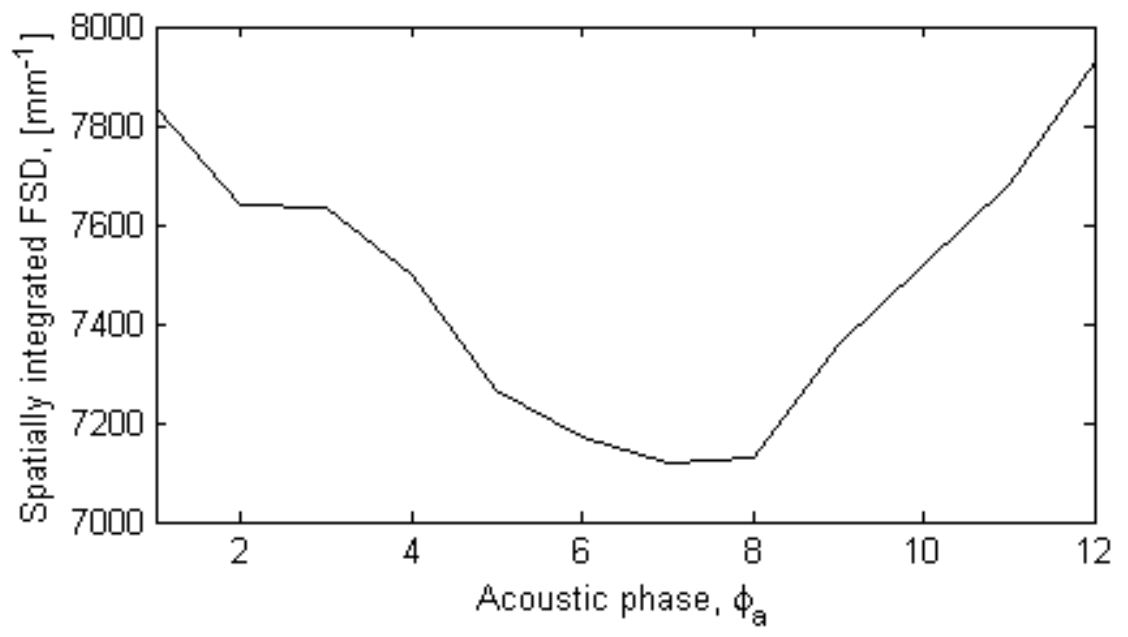

Figure 4.4: Net flame surface density as a function of the acoustic phase. The net FSD fluctuates sinusoidally over the acoustic cycle.

decrease in FSD. This was not observed in the measurements, as seen by comparing Fig. 4.3(b) with Fig. 4.4, which shows the net FSD fluctuations over the acoustic cycle for the same experimental case. The random fluctuations in net FHSD do not show an inverse relationship to the net FSD, which is roughly sinusoidal over the acoustic cycle. Local periodic flame extinctions therefore were found to be negligible contributors to the overall heat release fluctuations, leaving variations in flame corrugations and large scale flame deformations as likely causes.

While it was difficult to decouple large scale flame deformations from smaller scale flame corrugations, analysis of doubly-phase-resolved FSD data indicated the presence of large scale deformation of the flame. Experimental results show that the mean position of the flame deformed considerably over the acoustic cycle. This can be seen in the images of Fig. 4.5, which show the doubly phase averaged flame surface density $\left(\bar{\Sigma}^{\text {ah }}\right)$ at $\phi_{\mathrm{h}}=4$ for the acoustic phase of lowest $\left(\phi_{\mathrm{a}}=1\right)$ and highest $\left(\phi_{\mathrm{a}}=7\right)$ spatially integrated FSD. The position at which the HVC intersected the measurement plane is shown using magenta swirling strength isocontours for spatial referencing. At $\phi_{\mathrm{a}}=1$, the region of high $\bar{\Sigma}^{\text {ah }}$ intersected the upper vortex intersection, whereas at $\phi_{\mathrm{a}}=7$ it was lifted above the vortex intersection, clearly indicating a large scale deformation of the flame. In fact, a detailed analysis of flow-flame dynamics for a loud and quiet case will show that the spatial distribution of $\Sigma^{\mathrm{a}}$ over the acoustic cycle can be explained by large scale flame deformations resulting from interactions with the HVC. 


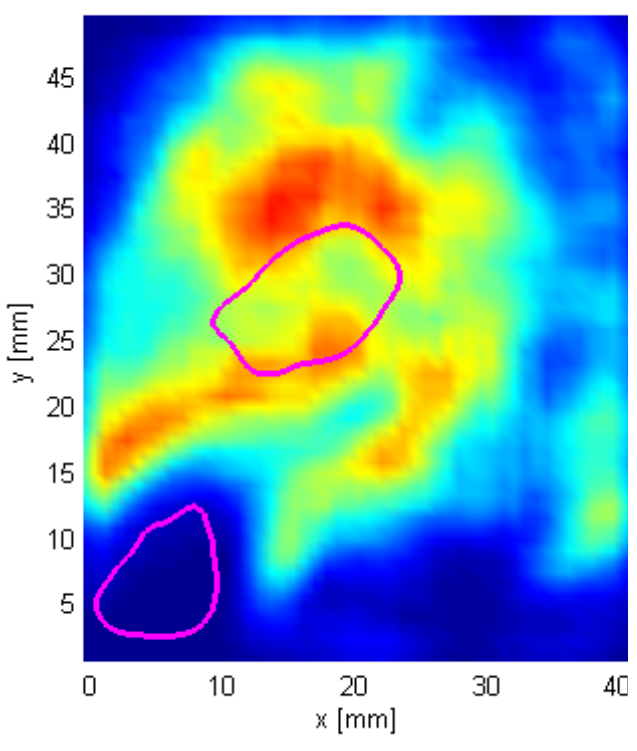

(a) $\phi_{\mathrm{a}}=1$.

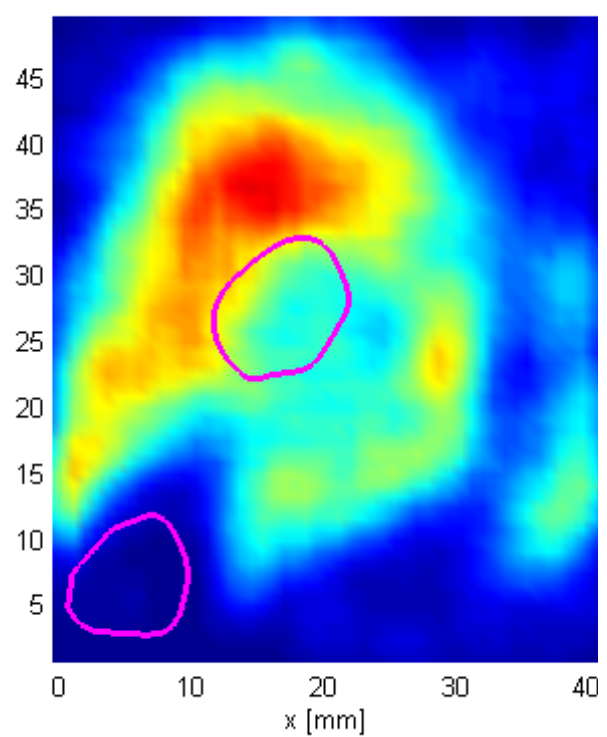

(b) $\phi_{\mathrm{a}}=7$.

Figure 4.5: Flame surface density slices taken at $\phi_{\mathrm{h}}=4$ of case 6 . Images correspond to the acoustic phase of lowest (a), and highest (b), spatially integrated flame surface density. At $\phi_{\mathrm{a}}=4$ the region of high FSD is low, intersecting an HVC intersection represented by a swirling strength isocontour. At $\phi_{\mathrm{a}}=7$, the region of high FSD is displaced above the vortex.

\subsubsection{Flame-HVC Interactions}

The large-scale flame deformations were associated with changes in the doubly-phaseresolved vorticity field ( viz. $\omega_{\mathrm{z}}^{\mathrm{a}}$ structures), which caused the flame to deform, periodically igniting reactants in the outer recirculation zone. These $\omega_{\mathrm{z}}^{\mathrm{a}}$ structures appeared as counter rotating vortex pairs that revolved around a common axis over the thermo-acoustic cycle. It is noted that these vortex pairs are observed in the doubly-phase-resolved vorticity fluctuation fields $\left(\omega_{z}^{\mathrm{a}}\right)$, and therefore they do not refer to the HVC, which appears in doubly-phase-resolved vorticity fields $\left(\bar{\omega}_{\mathrm{z}}^{\mathrm{ah}}\right)$. The $\omega_{\mathrm{z}}^{\mathrm{a}}$ structures do not represent physical structures in the flow, but provide an important means of visualizing and understanding the mechanisms driving acoustically-coupled flame oscillations. 


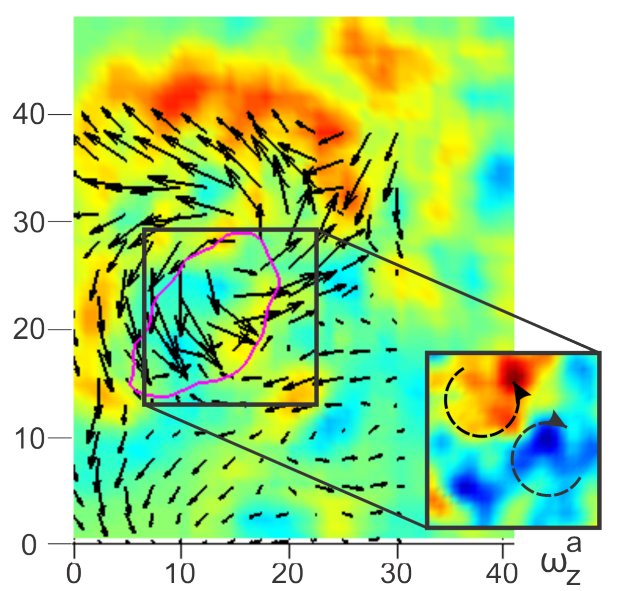

(a) $\phi_{\mathrm{a}}=1$.

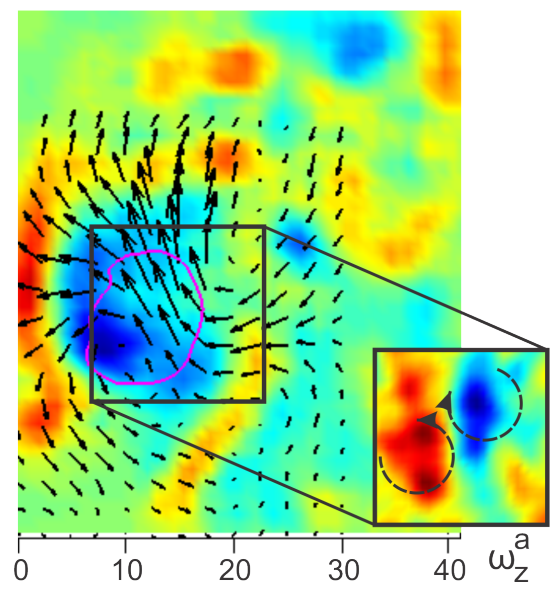

(b) $\phi_{\mathrm{a}}=4$.

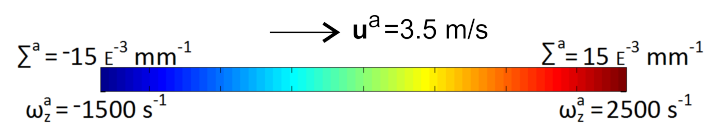

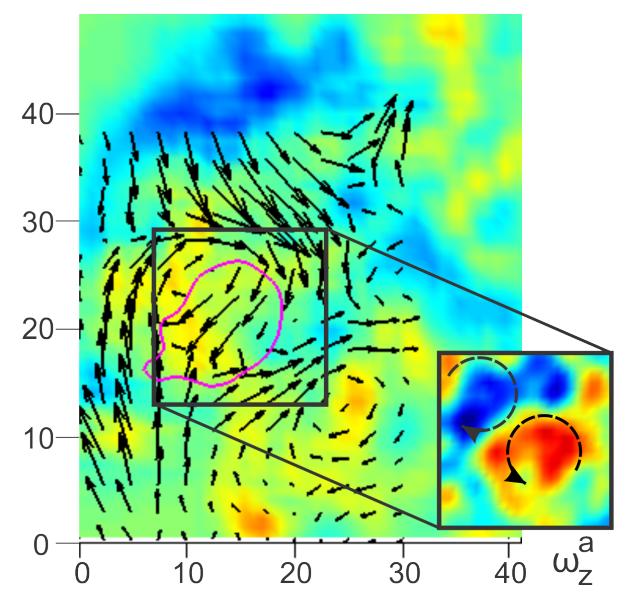

(c) $\phi_{\mathrm{a}}=8$.

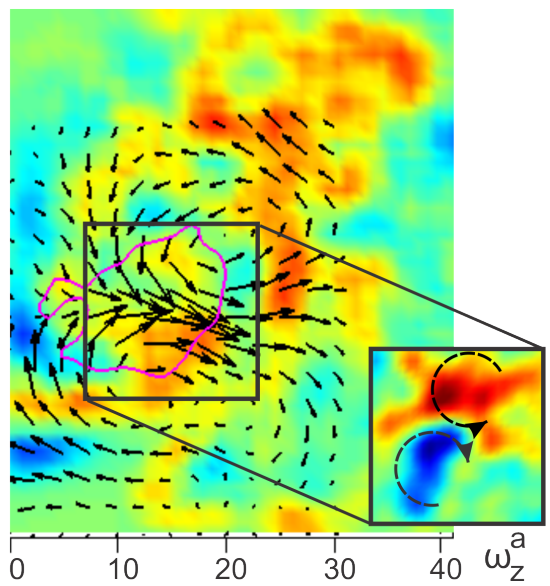

(d) $\phi_{\mathrm{a}}=11$.

Figure 4.6: Evolution of flame surface density fluctuations $\left(\Sigma^{\mathrm{a}}\right)$ over the acoustic cycle and velocity fluctuations $\left(\mathbf{u}^{\mathrm{a}}\right)$ at $\phi_{\mathrm{h}}=1$ for Case 6 . The HVC is shown by magenta iso-contours of swirl strength at $30 \%$ of the maximum swirl strength value. The fly-outs show $\omega_{\mathrm{z}}^{\mathrm{a}}$ in the vicinity of the HVC. Axis units are milimeters. 


\section{Role of the $\omega_{\mathrm{z}}^{\mathrm{a}}$ Structures in the Loudest Case}

The process by which the $\omega_{\mathrm{z}}^{\mathrm{a}}$ structures deformed the flame is illustrated in Fig. 4.6, which shows the doubly-phase-resolved flame surface density fluctuation $\left(\Sigma^{\mathrm{a}}\right)$ fields with superimposed velocity fluctuation vector fields $\left(\mathbf{u}^{\mathrm{a}}\right)$ in the loudest flame (Case 6). The data are shown at different stages in the acoustic cycle (different $\phi_{\mathrm{a}}$ ) and at a characteristic azimuthal position of the HVC $\left(\phi_{\mathrm{h}}=1\right)$. The location at which the HVC intersected the measurement plane is shown by a swirling strength iso-contour taken at $30 \%$ of the maximum value. Fly-outs in each subfigure show local vorticity fluctuation fields $\left(\omega_{\mathrm{z}}^{\mathrm{a}}\right)$ around the HVC. This figure shows how the flame and flow varied over the acoustic cycle relative to their acoustic-mean distributions.

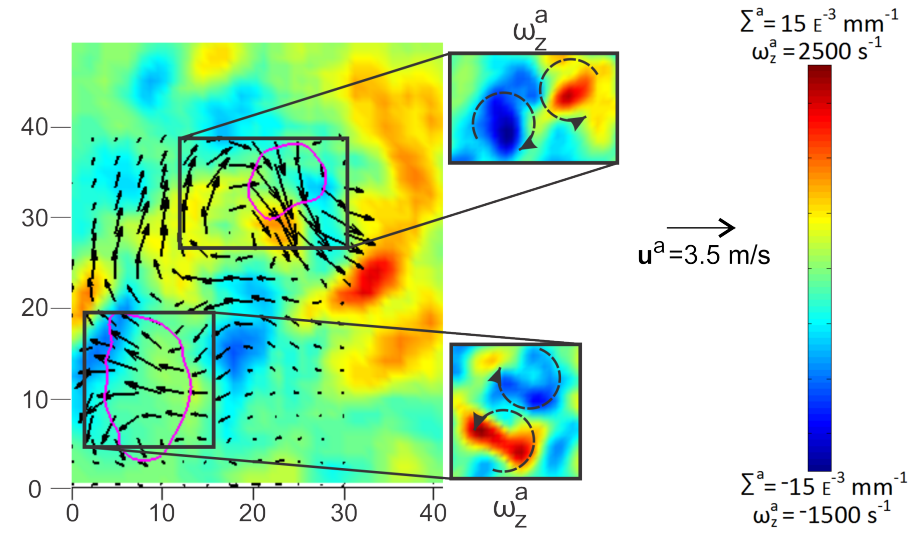

(a) $\phi_{\mathrm{a}}=1$.

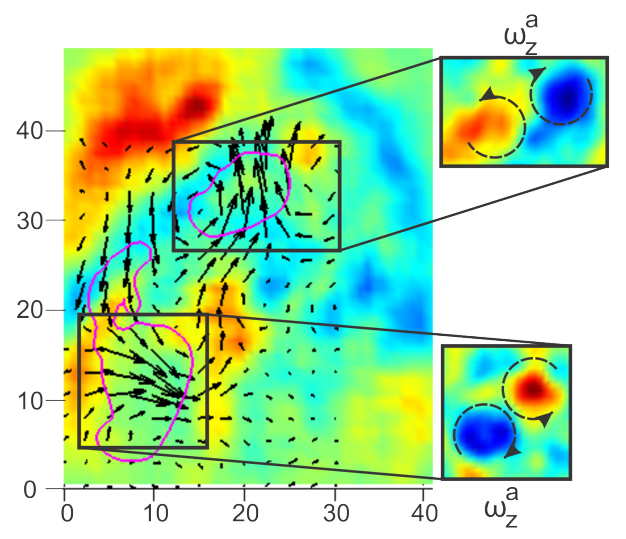

(b) $\phi_{\mathrm{a}}=7$.

Figure 4.7: Evolution of flame surface density fluctuations $\left(\Sigma^{\mathrm{a}}\right)$ over the acoustic cycle and velocity fluctuations $\left(\mathbf{u}^{\mathrm{a}}\right)$ at $\phi_{\mathrm{h}}=7$ for Case 6 . The HVC is shown by magenta iso-contours of swirl strength at $30 \%$ of the maximum swirl strength value. The fly-outs show $\omega_{\mathrm{z}}^{\mathrm{a}}$ in the vicinity of the HVC. Axis units are milimeters.

At $\phi_{\mathrm{a}}=1$, the $\omega_{\mathrm{z}}^{\mathrm{a}}$ structures were positioned such that the clockwise rotating vortex was below and slightly to the right of the counter-clockwise rotating vortex. This created a local velocity fluctuation towards the top part of the OCR, displacing and deforming the flame in this direction and causing positive $\Sigma^{\mathrm{a}}$ on top of the HVC intersection. At $\phi_{\mathrm{a}}=4$, the relative positions of the $\omega_{\mathrm{z}}^{\mathrm{a}}$ structures had rotated by approximately 90 degrees, with the clockwise rotating vortex now lying slightly higher and to the right of the counterclockwise rotating vortex. The associated velocity fluctuations pushed the flame upward 
and toward the ICR (Fig. 4.6(b)). Later in the acoustic cycle $\left(\phi_{\mathrm{a}}=8\right)$, the $\omega_{\mathrm{z}}^{\mathrm{a}}$ structures' relative positions had rotated by another 90 degrees. This pushed the flame downwards, resulting in the most contracted state of the flame in the acoustic cycle for this particular HVC phase, causing low flame surface area and very little positive $\Sigma^{\mathrm{a}}$, as seen in Fig. 4.6(c). At $\phi_{\mathrm{a}}=11$, the $\omega_{\mathrm{z}}^{\mathrm{a}}$ structures rotated by approximately another 90 degrees and the associated velocity fluctuations pushed the flame toward the OCR, igniting unburned reactants and increasing flame surface density in the process. Subsequently, these flames, assisted by the velocity fluctuations imparted by the $\omega_{\mathrm{z}}^{\mathrm{a}}$ structures, were moved upwards and towards the ICR until the state of $\phi_{\mathrm{a}}=1$ was reached at the beginning of the next cycle.

The same type of flow-flame dynamics were observed at azimuthal positions where the HVC intersected the measurement plane at two locations. However, a second $\omega_{\mathrm{z}}^{\mathrm{a}}$ structure appeared, such that a counter-rotating vortex pair was present in the vicinity of each HVC intersection. Figure 4.7 shows the same quantities as the sequence of Fig. 4.6, but for $\phi_{\mathrm{h}}=7$. At the acoustic phase of highest pressure $\left(\phi_{\mathrm{a}}=1\right)$, the $\omega_{\mathrm{z}}^{\mathrm{a}}$ structures near the top and bottom HVC intersections were out of phase by approximately 180 degrees, such that the flame was pushed into the OCR near the upper intersection and towards the burner axis near the lower intersection. This resulted in the creation of positive $\Sigma^{\mathrm{a}}$ in these areas. At the acoustic phase of lowest pressure $\left(\phi_{\mathrm{a}}=7\right)$, both $\omega_{\mathrm{z}}^{\mathrm{a}}$ structures had rotated by 180 degrees. This caused the flame to be pushed in the opposite directions to those occurring at $\phi_{\mathrm{a}}=1$. At the top vortex intersection, this led to negative $\Sigma^{\mathrm{a}}$ in the OCR and positive $\Sigma^{\mathrm{a}}$ in the ICR, as the flame was pushed back over the vortex intersection. On the other hand, the flame in the vicinity of the lower vortex intersection was pushed outwards, creating positive $\Sigma^{\mathrm{a}}$; this likely is actually a flame transfer from the other half of the burner.

\section{Role of the $\omega_{\mathrm{z}}^{\mathrm{a}}$ Structures in the Quietest Case}

The behaviour of the HVC-flame interactions in the quietest case were very similar to those of the loudest case, wherein $\omega_{\mathrm{z}}^{\mathrm{a}}$ structures in the form of counter-rotating vortices moved the flame back and forth between the ICR and the OCR over the acoustic cycle. To highlight the subtle differences in flow-flame interactions between the two cases, the analogous image sequence to Fig. 4.6 is reproduced in Fig. 4.8 for the quietest case. 
For a representative comparison, $\phi_{\mathrm{h}}$ was chosen so that the HVC intersection was at the same axial location as the one in Fig. 4.6. Because the spreading angle of the HVC in the quietest case was slightly different than the one in the loudest case, Fig. 4.8 is shown for $\phi_{\mathrm{h}}=2$ instead of $\phi_{\mathrm{h}}=1$.

As for the loudest case, the $\omega_{\mathrm{z}}^{\mathrm{a}}$ structures rotated about themselves once over the acoustic cycle; a counter-clockwise rotation of approximately 90 degrees was observed over each acoustic phase increment in Fig. 4.8. The associated velocity fluctuations pushed the flame back and forth between the OCR and the ICR. The most significant difference in observed behaviour between the $\omega_{\mathrm{z}}^{\mathrm{a}}$ structures in the quietest case and those of the loudest case was a notable phase shift in their rotation. That is, the counterclockwise rotation of the $\omega_{\mathrm{z}}^{\mathrm{a}}$ structures in the quietest case lagged behind that of the loudest case. This also was true of the associated velocity fluctuations, and hence the flame's transition between the ICR and the OCR was also delayed. For example, whereas the velocity fluctuations pushed the flame above the HVC and into the ICR at $\phi_{\mathrm{a}}=1$ in the loudest case (see Fig. 4.6(a)), the flame was pushed out into the OCR at the same acoustic phase of the quietest case (Fig. 4.8(a)). In fact, the flame did not transition to the ICR until later in the acoustic cycle, at $\phi_{\mathrm{a}}=4$. The flame was then deformed by the revolving $\omega_{\mathrm{z}}^{\mathrm{a}}$ structures; first towards the ICR at $\phi_{\mathrm{a}}=8$ and then downwards at $\phi_{\mathrm{a}}=11$, eventually igniting reactants in the OCR at $\phi_{\mathrm{a}}=1$. It is noted that the heat release dynamics described here are qualitatively the same as those found for the loudest case, with the sole difference being a phase shift relative to the acoustic cycle. It is therefore apparent that the increase in thermo-acoustic energy transfer from the quietest to the loudest case does not result from a change in the fundamental nature of the flow-flame interactions, but rather from a change in the phase at which these interactions occur. Since the heat release dynamics were set by the $\omega_{\mathrm{z}}^{\mathrm{a}}$ structures, a thorough understanding of the thermo-acoustic velocity coupling in the model combustor requires an understanding of the processes that create such structures, and set their phase relative to the acoustic cycle.

\subsubsection{Origin of the $\omega_{\mathrm{z}}^{\mathrm{a}}$ structures}

From the discussion above, it is clear that the $\omega_{\mathrm{z}}^{\mathrm{a}}$ structures are crucial in setting the spatial distribution of the $\Sigma^{\mathrm{a}}$ fields, and hence the thermo-acoustic energy transfer. Un- 


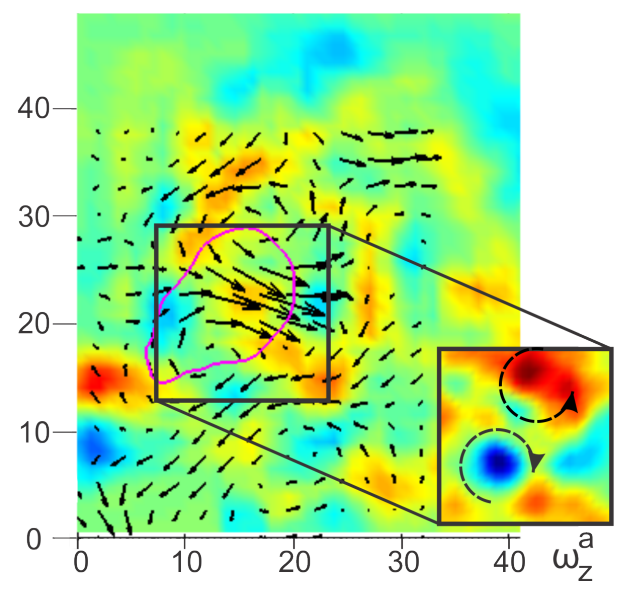

(a) $\phi_{\mathrm{a}}=1$.

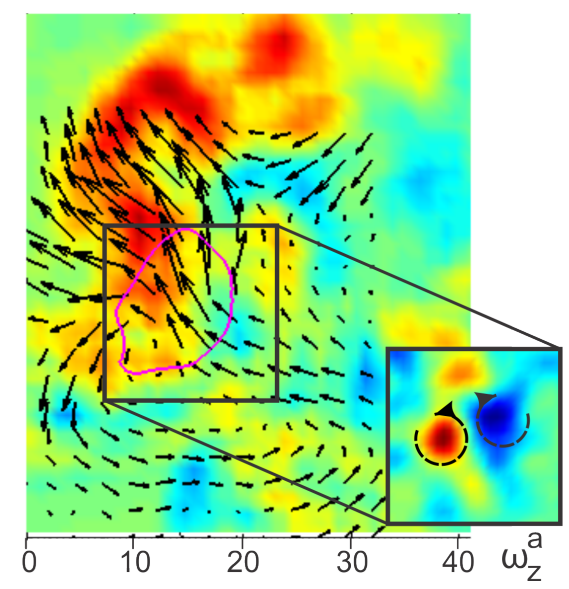

(b) $\phi_{\mathrm{a}}=4$.

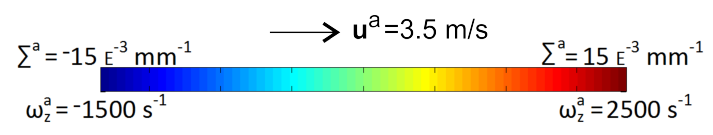

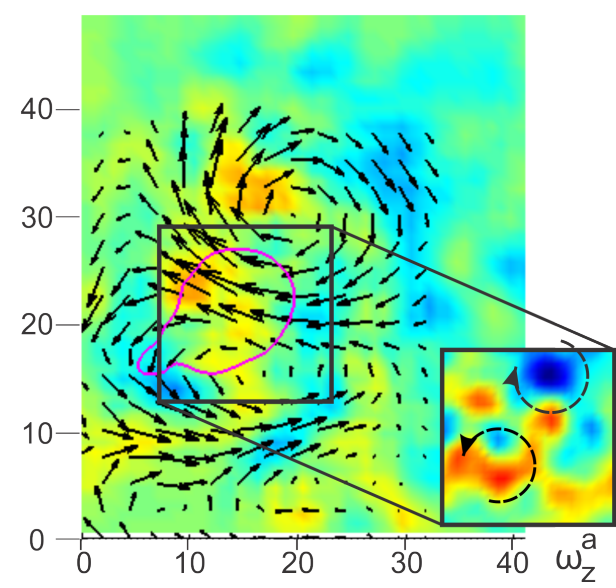

(c) $\phi_{\mathrm{a}}=8$.

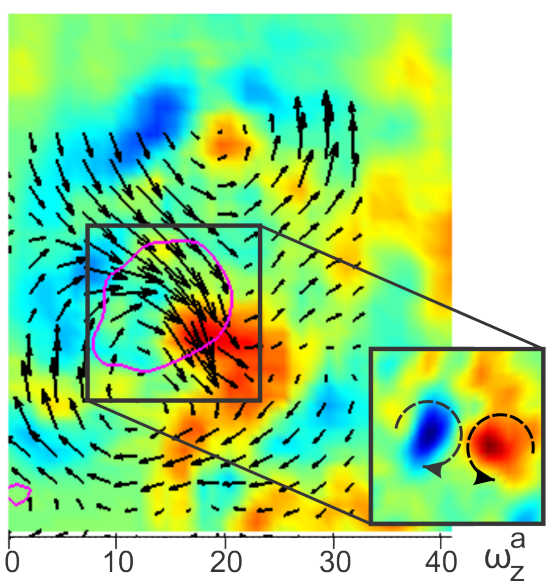

(d) $\phi_{\mathrm{a}}=11$.

Figure 4.8: Evolution of flame surface density fluctuations $\left(\Sigma^{\mathrm{a}}\right)$ over the acoustic cycle and velocity fluctuations $\left(\mathbf{u}^{\mathrm{a}}\right)$ at $\phi_{\mathrm{h}}=2$ for Case 6 . The HVC is shown by magenta iso-contours of swirl strength at $30 \%$ of the maximum swirl strength value. The fly-outs show $\omega_{\mathrm{z}}^{\mathrm{a}}$ in the vicinity of the HVC. Axis units are milimeters. 
derstanding the $\psi$ distributions therefore requires an understanding of what creates these structures and why they revolve over the acoustic cycle. The origin of the $\omega_{\mathrm{z}}^{\mathrm{a}}$ structures was found to be a dynamic deformation of the HVC over the thermoacoutic cycle. That is, at each HVC phase, the position at which the HVC intersected the measurement plane changed over the acoustic cycle. This is illustrated in the cartoon of Fig. 4.9, which shows the red HVC's intersection with the measurement plane. A white cross marks the swirling-strength weighted doubly-phase-resolved centroid $\left(\bar{x}_{\mathrm{c}}^{\mathrm{ah}}, \bar{y}_{\mathrm{c}}^{\mathrm{ah}}\right)$ of each HVC intersection, which were computed over the region around the local swirling-strength peaks corresponding to values greater than half the local maximum. The deformation of the HVC in Fig. 4.9 is exaggerated for clarity, and the actual vortex intersection displacements calculated from $\bar{x}_{\mathrm{c}}^{\text {ah }}$ and $\bar{y}_{\mathrm{c}}^{\text {ah }}$ are shown in Fig. 4.10 for different HVC phases. To understand how a displacement in the HVC intersection affected the $\omega_{\mathrm{z}}^{\mathrm{a}}$ structures, it is useful to consider a simple unidirectional deformation of the HVC. As the HVC moves, for example, from left to right, the region of high vorticity also is displaced. Hence, negative $\left(\omega_{\mathrm{z}}^{\mathrm{a}}<0\right)$ and positive $\left(\omega_{\mathrm{z}}^{\mathrm{a}}>0\right)$ oscillations would occur to the left and right of the mean HVC position, respectively. The previously noted rotation of the $\omega_{z}^{\mathrm{a}}$ structures around a common axis was therefore a result of the elliptical trajectory of the HVC intersection, observed as a phase lag between the axial and radial displacements in Fig. 4.10.

The cause of the HVC deformation is thought to be a swirl number wave that propagated from the burner nozzle associated with an axial velocity wave, caused by the changing pressure difference between the chamber and plenum. This wave convected downstream and interacted with the HVC. Such convective bulk velocity waves have been observed in the past by several researchers [106]. Here, they were observed via the cross-section integrated axial velocity fluctuations $\left(\left\langle u_{\mathrm{y}}\right\rangle^{\mathrm{a}}\right.$, refer to Sec. 3.3), shown in Fig. 4.11 for different acoustic phases of Case 6. It was first hypothesised that the mechanism by which the $\left\langle u_{\mathrm{y}}\right\rangle^{\mathrm{a}}$ wave deformed the HVC was convection. In this case, the displacement of the vortex anywhere along the helix would obey the well known classical relation that displacement is the temporal integral of velocity. To test this, the displacement of the HVC's intersection at $\phi_{\mathrm{h}}=1$ was compared to the displacement that would have resulted from convection of the HVC by the $\left\langle u_{\mathrm{y}}\right\rangle^{\mathrm{a}}$ wave. The latter was calculated by integrating the velocity fluctuations over the acoustic cycle, taken at the acoustic-mean height of the HVC's centroid $\left(y=\overline{\bar{y}_{c}^{\mathrm{ah}}}\left(\phi_{\mathrm{h}}=1\right)\right)$. The measured deformations, on the other hand, were computed directly from the centroid locations $\bar{y}_{c}^{\text {ah }}$. Fig. 4.12 shows 


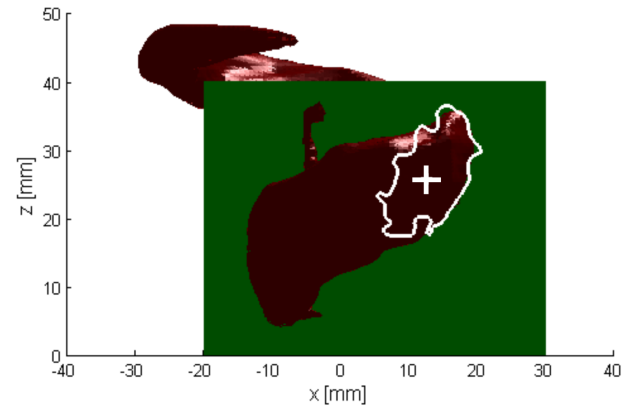

(a) $\phi_{\mathrm{a}}=1$.

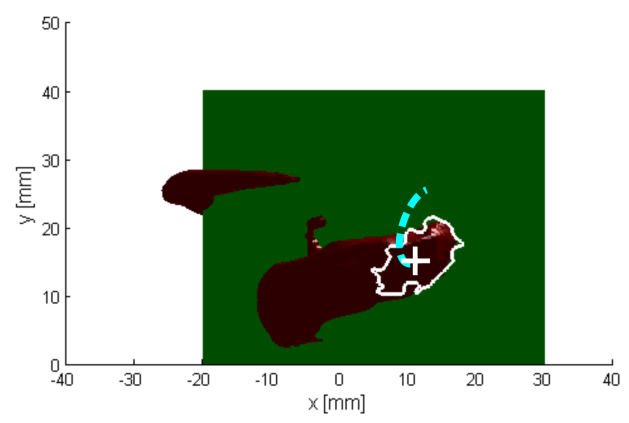

(c) $\phi_{\mathrm{a}}=8$.

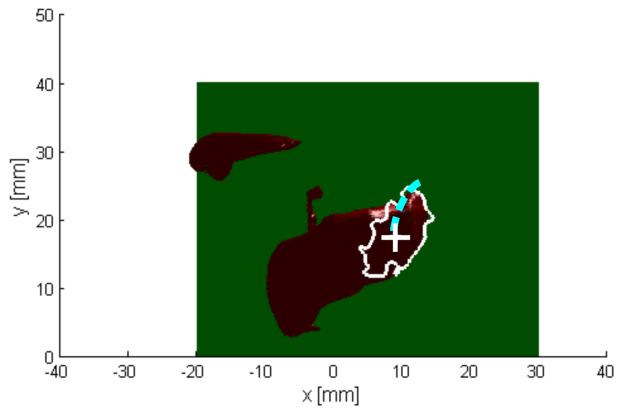

(b) $\phi_{\mathrm{a}}=4$.

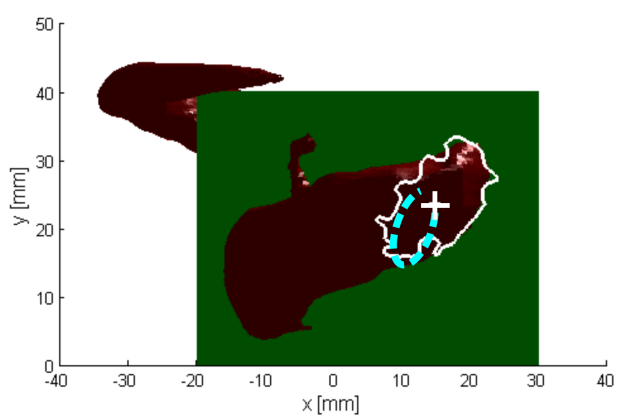

(d) $\phi_{\mathrm{a}}=11$.

Figure 4.9: Illustration of the HVC's deformation over the acoustic cycle. The HVC is shown as a red vorticity isosurface $\left(\omega_{\mathrm{z}}=5000 \mathrm{~s}^{-1}\right)$ and the PIV measurement plane is shown in green. The intersection of the HVC and the measurement plane is shown in

white, with a white cross marking the location of the swirling-strength weighted centroid of the vortex intersection. The elliptical trajectory of the HVC intersection is depicted by a dashed blue line, tracing the location of the vortex intersection's centroid over the acoustic cycle.

that both the phase and the amplitude of the HVC's measured deformation at $\phi_{\mathrm{h}}=1$ were quite different from that would have resulted from convection. This implies that convection was not responsible for the deformation of the HVC.

Rather, the $\left\langle u_{\mathrm{y}}\right\rangle^{\mathrm{a}}$ wave influenced the shape of the HVC through fluctuations in the swirl number, a critical parameter in the formation of HVCs (see Sec. 1.5). Performing Reynold's decomposition of the axial velocity component, $u_{\mathrm{y}}=\bar{u}_{\mathrm{y}}+u_{\mathrm{y}}^{\prime}$, where $\bar{u}_{\mathrm{y}}$ is the time averaged axial velocity and $u_{\mathrm{y}}^{\prime}$ is the fluctuating component, the instantaneous swirl 


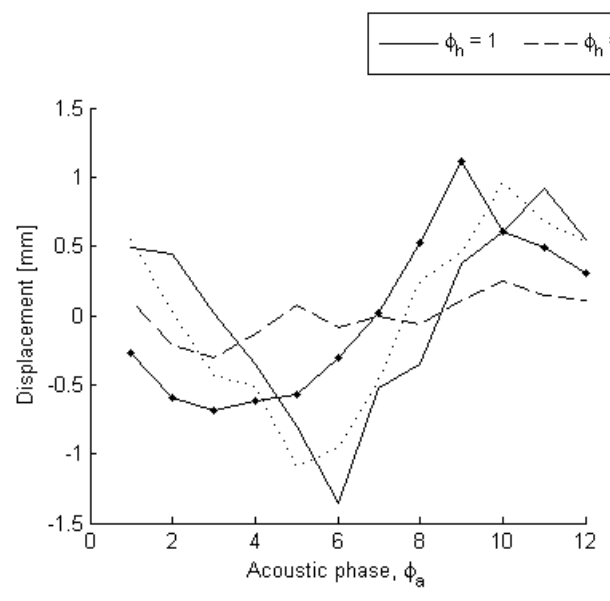

(a) Axial displacement.

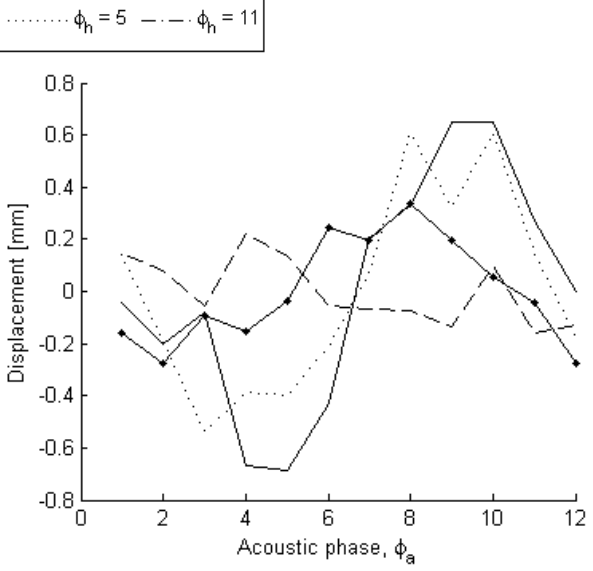

(b) Radial displacement.

Figure 4.10: Axial and radial displacement of the vortex intersection for Case 6 over the acoustic cycle.

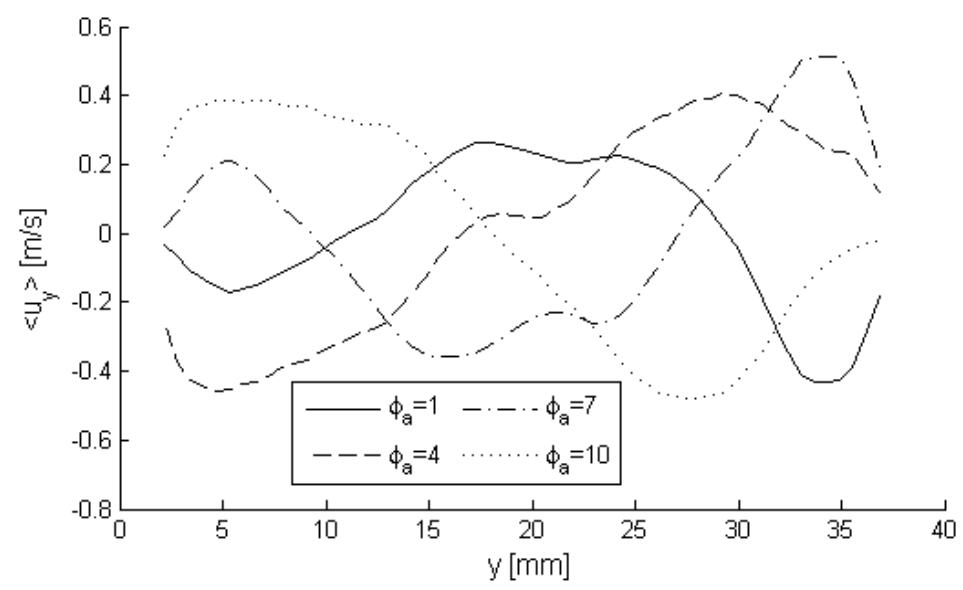

Figure 4.11: Axial velocity wave $\left(\left\langle u_{\mathrm{y}}\right\rangle^{\mathrm{a}}\left(\phi_{\mathrm{a}}\right)\right)$ as a function of axial location at different acoustic phases.

number can be written as [19]

$$
S=\bar{S}\left(1-\frac{u_{\mathrm{y}}^{\prime}}{\bar{u}_{\mathrm{y}}}\right) .
$$

This expression describes the relationship between the instantaneous swirl number and the velocity fluctuations of a given flow field: $S$ reaches its minimum value when $u_{\mathrm{y}}^{\prime}$ is at its maximum. For sinusoidal fluctuations, this entails a phase shift of 180 degrees, which was indeed found to be case. This phase shift is seen in Fig. 4.13, which shows $\left\langle u_{\mathrm{y}}\right\rangle^{\mathrm{a}}$ and $\langle S\rangle^{\mathrm{a}}$ as a function of axial distance from the burner nozzle at $\phi_{\mathrm{a}}=1$. Such swirl number 
waves were also noticed by Palies et al. [19, 106], and are thought to affect the shape of the HVC through a displacement of the central recirculation zone.

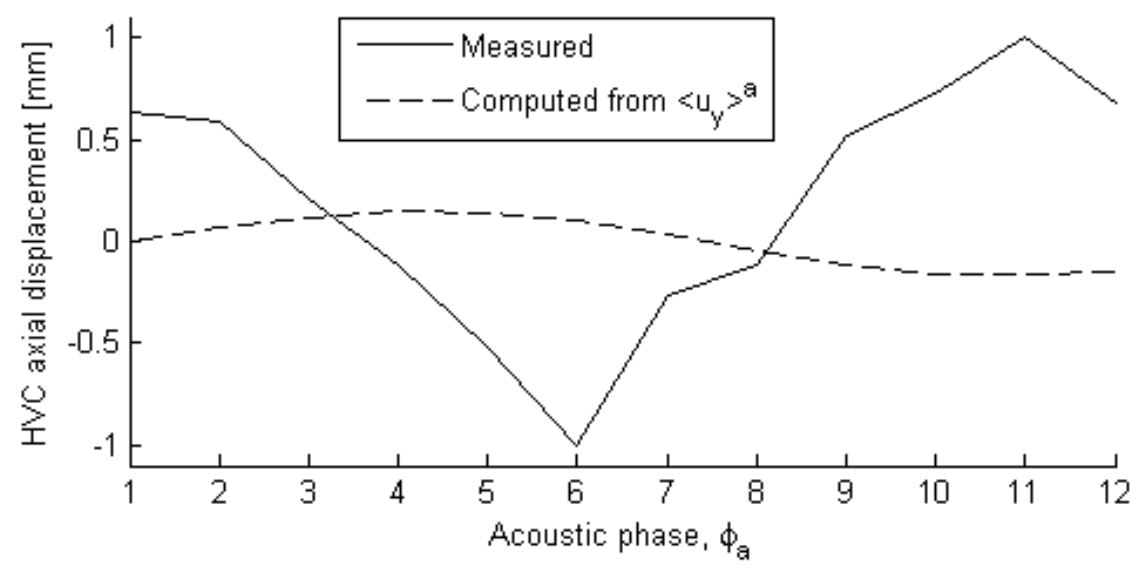

Figure 4.12: Comparison of the experimental vortex intersection displacement at $\phi_{\mathrm{h}}=1$ with the displacement that would result from convection by the $\langle a\rangle_{\mathrm{y}}^{\mathrm{a}}$ waves. The disagreement in amplitude and phase indicates that the HVC deformation was not due to convection of the vortex by the axial velocity wave.

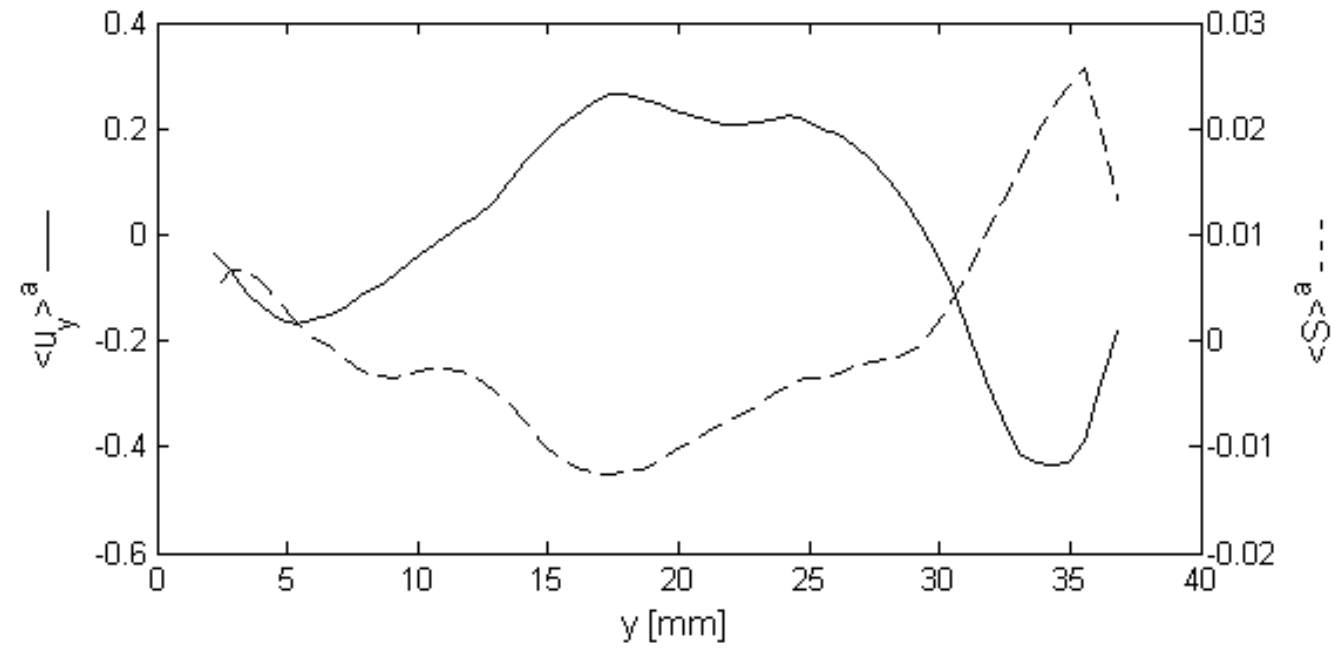

Figure 4.13: Axial distribution of $\left\langle u_{\mathrm{y}}\right\rangle^{\mathrm{a}}$ and $\langle S\rangle^{\mathrm{a}}$ at $\phi_{\mathrm{a}=1}$ for case 6. Local swirl number fluctuations are imparted by axial velocity fluctuations, with a 180 degree phase shift in the two waves resulting from Eq. 4.3.

Palies et al. also observed that the swirl wave was convected by the mean flow. To verify this, the convective speed of the swirl wave was computed by fitting a line to the $\langle S\rangle^{\mathrm{a}}$ weighted space-phase domain in which the swirl wave evolved. This domain is 


Case Mean axial flow velocity $[\mathrm{m} / \mathrm{s}] \quad$ Swirl wave convective velocity $[\mathrm{m} / \mathrm{s}] \quad$ Disagreement $[\%]$

$\begin{array}{cccc}3 & 8.0 & 8.7 & 9 \\ 4 & 9.2 & 9.6 & 4 \\ 5 \mathrm{~b} & 11.1 & 11.3 & 2 \\ 6 & 12.9 & 13.4 & 4 \\ 7 & 11.3 & 11.3 & 0\end{array}$

Table 4.1: Comparison of the mean axial flow velocity and the convective speed of the swirl wave.

plotted in Fig. 4.14, with the colormap indicating $\langle S\rangle^{\mathrm{a}}$, and a black line showing the linear curve fit. The convective speed of the wave was calculated from $\Delta y n_{\phi_{\mathrm{a}}} f_{\mathrm{ac}} / \Delta \phi_{\mathrm{a}}$, where $n_{\phi_{\mathrm{a}}} f_{\mathrm{ac}}$ is a constant included to convert from phase space to time. This speed is compared to the mean axial flow velocity in the combustor, which was extracted from the PIV measurements of the different cases. This comparison appears in Table 4.1, where it is concluded that the swirl wave did indeed propagate at the mean flow velocity, with an uncertainty of less than $9 \%$.

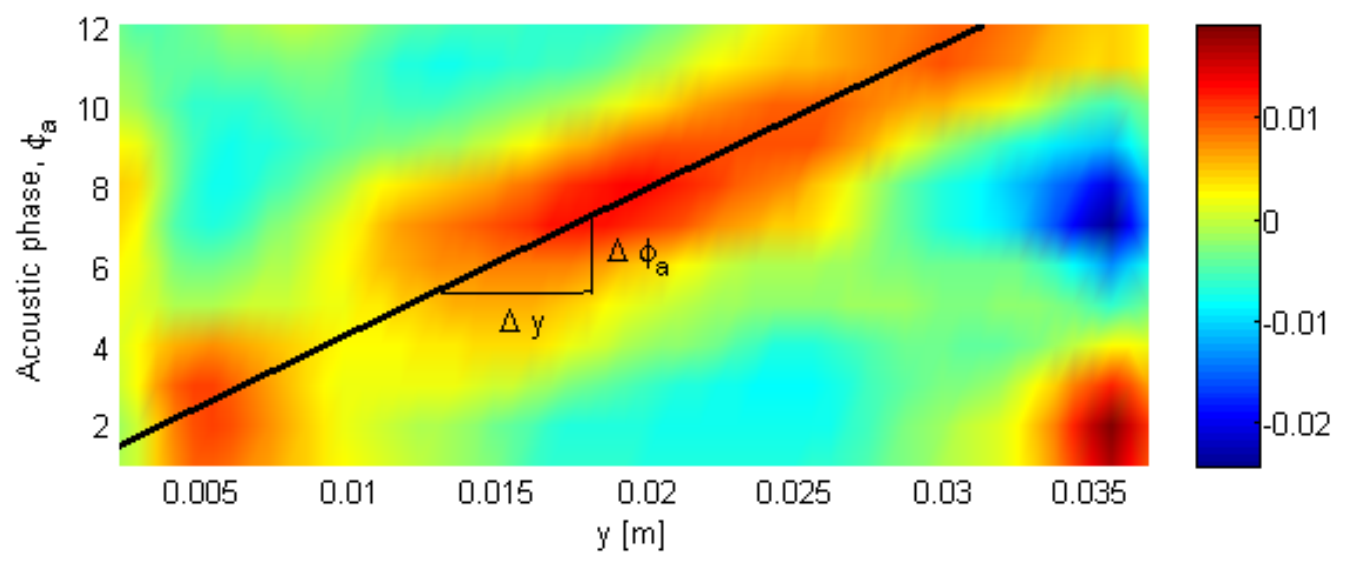

Figure 4.14: Swirl fluctuation plotted as a function of axial position and the acoustic cycle. The convective speed of the $\langle S\rangle^{\mathrm{a}}$ wave was found by fitting a line through the $\langle S\rangle^{\mathrm{a}}$ weighted domain. The speed can be evaluated by taking the reciprocal of the line's slope. 


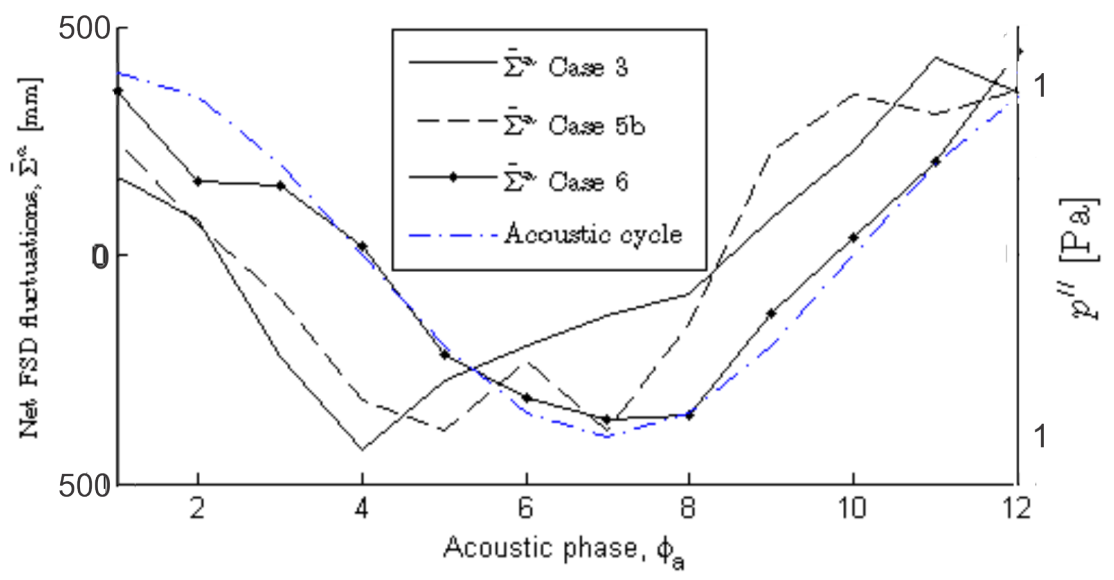

Figure 4.15: Fluctuations of the spatially integrated flame surface density $\left(\tilde{\Sigma}^{\mathrm{a}}\right)$ over the acoustic cycle for the quietest case (3), an intermediate case (5b), and the loudest case (6). The acoustic cycle is shown as a dash-doted line for phase referencing. As the cases get louder, $\tilde{\Sigma}^{\mathrm{a}}$ becomes gradually more in phase with the acoustics, increasing thermo-acoustic energy transfer.

\subsection{Phase Relationships}

As shown in Sec. 4.2, velocity fluctuations periodically deformed the flame front, cyclically igniting reactants in the OCR. While the velocity coupling mechanism was similar for all cases, the phase relative to the acoustic cycle at which the flame underwent transition from the ICR to the OCR changed as a function of combustor conditions and $\phi_{\mathrm{h}}$. As a result, the spatially integrated FSD fluctuations $\left(\tilde{\Sigma}^{\mathrm{a}}\right)$ also changed in phase relative to the acoustic cycle. The flame's transition from the ICR to the OCR was caused by a deformation of the HVC induced by a swirling strength wave that propagated from the burner nozzle, and therefore the phase of $\tilde{\Sigma}^{\mathrm{a}}$ relative to the acoustic cycle $\left(\phi_{\tilde{\Sigma}^{\mathrm{a}}-a}\right)$ may be expressed as the combination of a phase lag between the acoustics, the swirling strength wave, and $\tilde{\Sigma}^{\mathrm{a}}$ via the relation

$$
\phi_{\tilde{\Sigma}^{\mathrm{a}}-a}=\phi_{\langle S\rangle^{\mathrm{a}}-a}+\phi_{\tilde{\Sigma}^{\mathrm{a}}-\langle S\rangle^{\mathrm{a}}}
$$

where $\phi_{\langle S\rangle^{a}-a}$ is the phase between the swirling strength fluctuations at the burner nozzle and the acoustic cycle, and $\phi_{\tilde{\Sigma}^{a}-\langle S\rangle^{a}}$ is the phase between the spatially integrated FSD fluctuations and the swirling strength fluctuations at the burner nozzle. 
The phase relationship between $\tilde{\Sigma}^{\mathrm{a}}$ and the acoustic cycle can be qualitatively assessed from Fig. 4.15, which shows the evolution of $\tilde{\Sigma}^{\mathrm{a}}$ over the acoustic cycle for cases $3,5 \mathrm{~b}$, and 6 , along with a scaled acoustic signal for phase referencing. While the amplitude of $\tilde{\Sigma}^{\mathrm{a}}$ did not change much between cases, it became increasingly in phase with the acoustics cycle for the louder cases. For a rough estimation of the phase difference between the the acoustic cycle and the spatially integrated FSD, one may compare the minima of the functions. For example, $\tilde{\Sigma}^{\mathrm{a}}$ for case 3 reached a minimum at $\phi_{\mathrm{a}}=4$, whereas the pressure reached a minimum at $\phi_{\mathrm{a}}=7$ (by definition, see Sec. 3.3). Hence, for the 12segment phase decomposition performed in this analysis, $\tilde{\Sigma}^{\mathrm{a}}$ of case 3 was approximately $(7-4) \frac{360}{12}=90$ degrees out of phase with the acoustic cycle. This resulted in very low thermo-acoustic energy transfer and low acoustic amplitudes. A similar analysis for case 6 indicated that $\tilde{\Sigma}^{\mathrm{a}}$ was perfectly in phase with the acoustic cycle, resulting in extremely favourable conditions for thermo-acoustic energy transfer and high amplitude acoustic oscillations. For a more accurate quantification of the phase lag between the acoustic cycle and $\tilde{\Sigma}^{\mathrm{a}}$, a co-sinusoid of the form $\zeta\left(\phi_{\mathrm{a}}\right)=A \cos \left(\omega \phi_{a}+\phi_{\tilde{\Sigma}^{\mathrm{a}}-a}\right)+B$ was fitted to the acoustically resolved data. The results are plotted with square markers for all experimental cases in Fig. 4.16(a), where the phase lag is shown as a function of the RMS pressure amplitude.
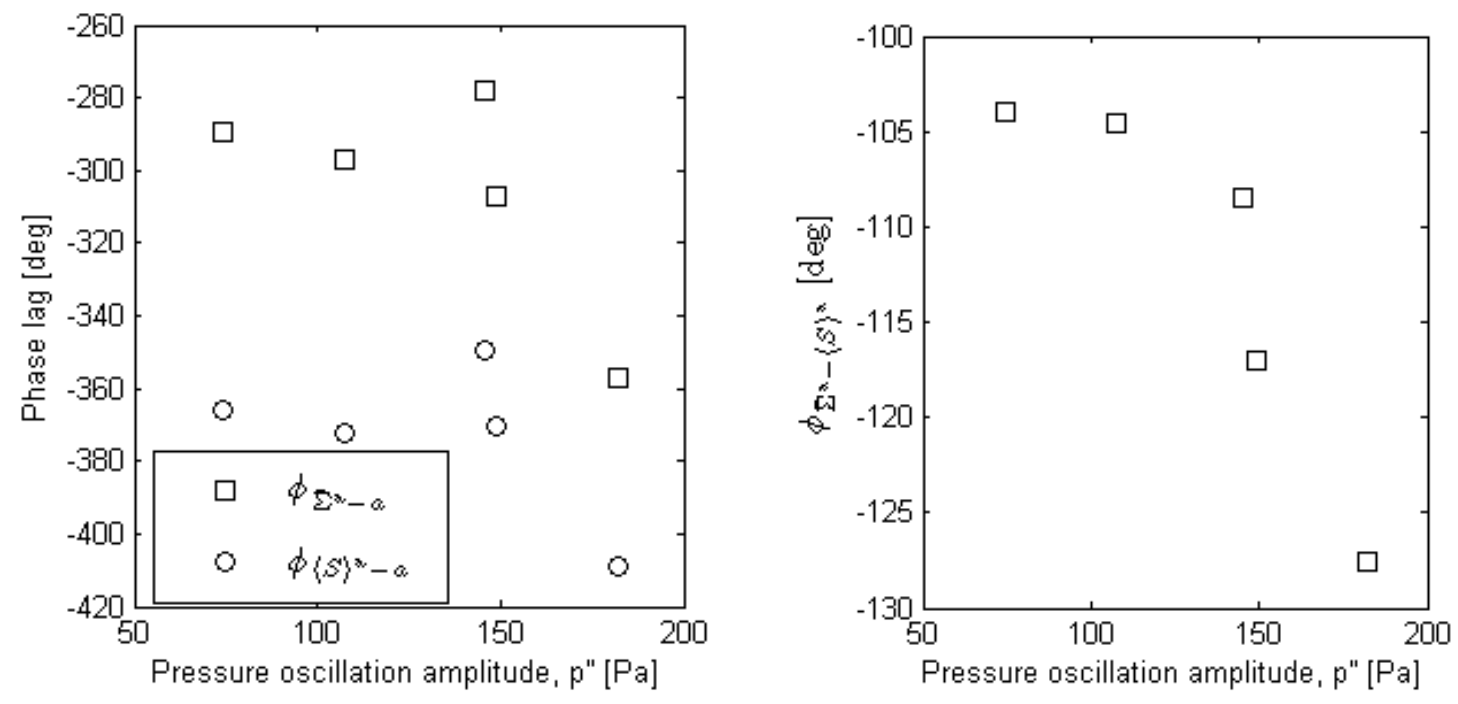

(a) Phase lag with respects to the acoustic cy- (b) Phase lag between $\langle S\rangle^{\mathrm{a}}\left(y=0, \phi_{\mathrm{a}}\right)$ and $\tilde{\Sigma}^{\mathrm{a}}$. cle of $\tilde{\Sigma}^{\mathrm{a}}$ and $\langle S\rangle^{\mathrm{a}}\left(y=0, \phi_{\mathrm{a}}\right)$.

Figure 4.16: Phase relationship between the acoustic cycle, $\tilde{\Sigma}^{\mathrm{a}}$ and $\langle S\rangle^{\mathrm{a}}\left(y=0, \phi_{\mathrm{a}}\right)$. 


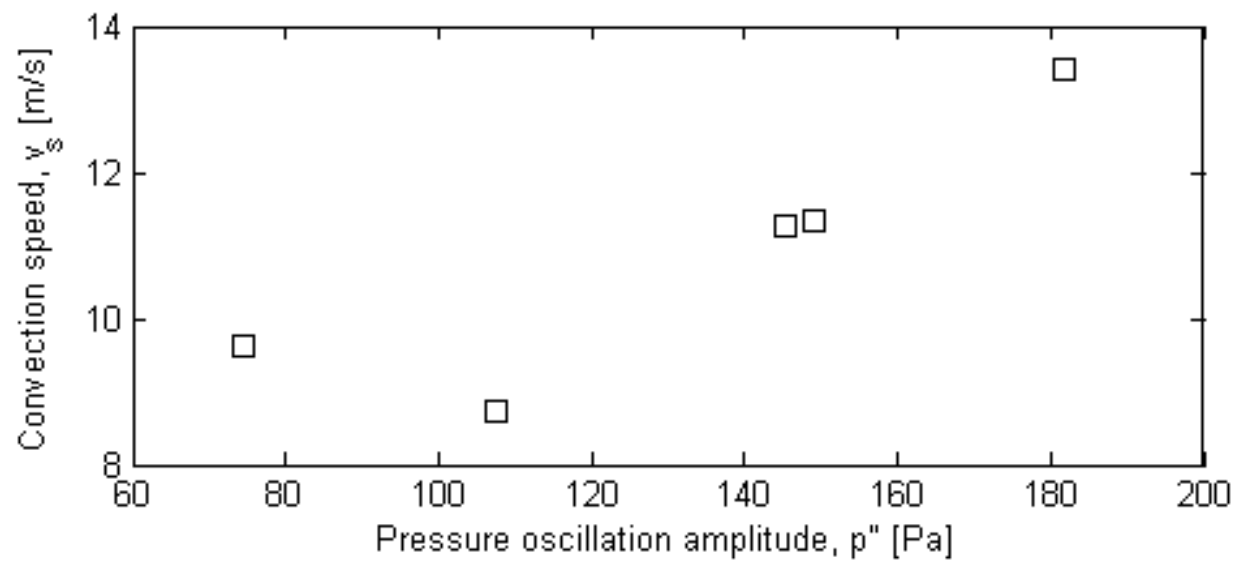

Figure 4.17: Convective speed of the swirling strength wave as a function of the RMS pressure amplitude.

Also plotted in this figure are the phase lags between the acoustic cycle and the swirl number wave at the nozzle exit plane $\left(\phi_{\langle S\rangle^{\mathrm{a}}-a}\right)$ for the corresponding experimental cases. These were obtained using the same co-sinusoid curve fitting approach outlined earlier, applied to $\langle S\rangle^{\mathrm{a}}\left(y=0, \phi_{\mathrm{a}}\right)$. The similar trend highlights the importance of the swirl number wave's influence on heat release oscillations, and shows that the change in phase of the heat release oscillations were largely due to a change in phase of the swirling strength wave.

The phase relationship between the FSD and swirling strength fluctuations was obtained from Eq. 4.4, and is illustrated as a function of the RMS pressure amplitude in Fig. 4.16(b). $\phi_{\tilde{\Sigma}^{\mathrm{a}}-\langle S\rangle^{\mathrm{a}}}$ represents the delay between the deformation of the HVC at the flame and the swirling strength fluctuations at the burner nozzle. Hence, the decreasing trend seen in Fig. 4.16(b) implies that the swirl number fluctuations at the nozzle exit were felt earlier at the flame for cases with larger pressure oscillation amplitudes. This phase relationship was determined by the convective speed of the swirl number wave, shown in Fig. 4.17. An increase in this speed meant that it took less time for the swirl number wave to convect downstream from the nozzle exit plane to the flame, thereby reducing the time and phase delay between the swirl number fluctuations at the nozzle and the total FSD oscillations.

From the discussion above, and from Eq. 4.4, the change in phase of the heat release oscillations relative to the acoustic cycle was mostly caused by a change in the phase and 


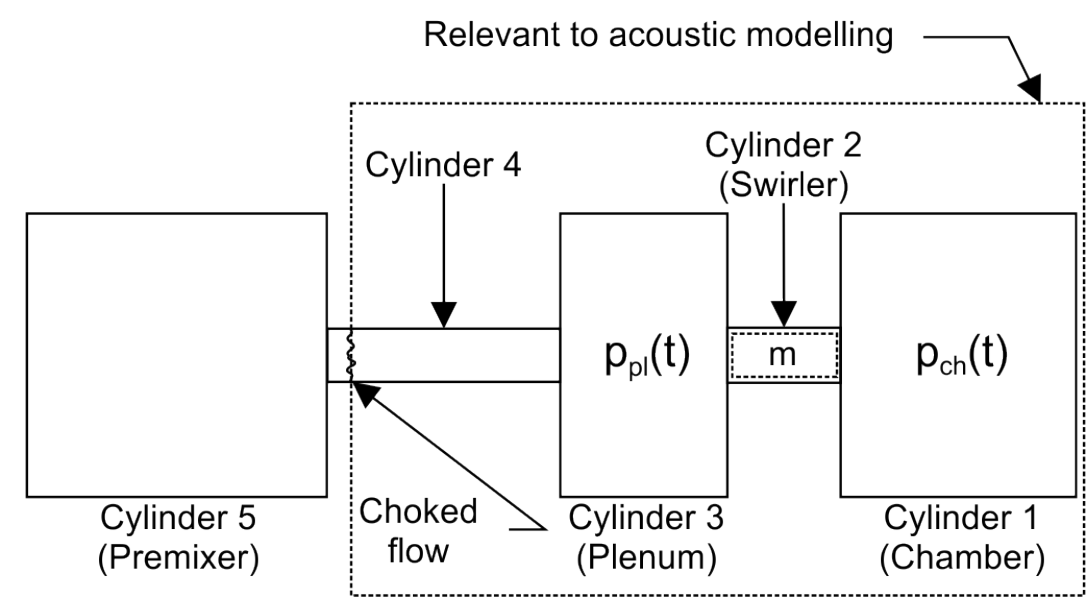

Figure 4.18: Simplified representation of the model combustor using a network of cylinders.

convective speed of the swirl number wave. While it has already been established that $\langle S\rangle^{\mathrm{a}}$ convected at the mean axial velocity in the combustor, understanding the phase lag between the acoustic cycle and the swirl number fluctuations at the nozzle exit $\left(\phi_{<S>-a}\right)$ requires further analysis.

\subsubsection{Quantifying $\phi\langle S\rangle^{\mathrm{a}}-a$}

The swirl number fluctuations may be quantified from Eq. 4.3. The fluctuations are obtained by expanding this equation, and keeping only the terms with fluctuating components. Replacing the instantaneous term $u_{\mathrm{y}}^{\prime}$ by its doubly phase resolved equivalent $\left\langle u_{\mathrm{y}}\right\rangle\left(y, \phi_{\mathrm{a}}\right)$, the swirl number fluctuations are

$$
\langle S\rangle\left(y, \phi_{\mathrm{a}}\right)=-\bar{S} \frac{\left\langle u_{\mathrm{y}}\right\rangle\left(y, \phi_{\mathrm{a}}\right)}{\bar{u}_{\mathrm{y}}} .
$$

Where as the mean swirl number and flow speed may be estimated from the mass flow rate of reactants and the geometry of the burner, estimating the axial velocity oscillations is more involved. Recall from Sec. 3.1 that the pressure within the model combustor was spatially uniform, meaning that the burner behaved like a multi-chamber Helmholtz resonator. In this case, the burner can be modelled as a spring-mass-damper system [107]. To quantify the behaviour of the combustor, its geometry was represented as a sequence of cylindrical chambers conserving the length, cross-sectional area, and volume of the different burner components, as illustrated in Fig. 4.18. To understand how this 


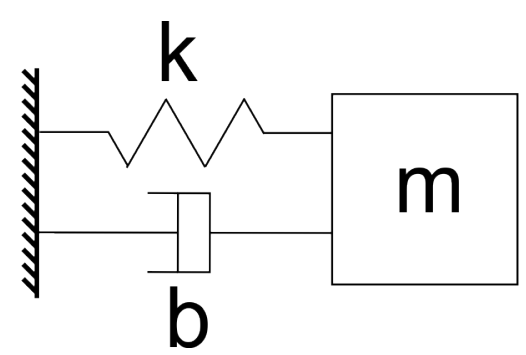

Figure 4.19: Spring-mass-damper model of the acoustic behaviour in the gas turbine model combustor.

system can properly represent the acoustic response of the model combustor, consider the representation of Fig. 4.18 to be completely quiescent. If the chamber pressure $\left(p_{\mathrm{ch}}\right)$ were to be suddenly increased, the pressure difference between the chamber and the plenum would induce a force on the fluid in the swirler, and it would uniformly accelerate towards the plenum. Hence, the fluid in the swirler can be replaced by a lumped mass. As this mass is displaced into the plenum, it compresses the gases according to the thermodynamic relation for isentropic compression [107]

$$
p_{\mathrm{pl}}=-\gamma \frac{\Delta V}{V_{\mathrm{pl}}} p_{0, \mathrm{pl}}
$$

where $p_{\mathrm{pl}}$ is the pressure in the plenum, $\gamma$ is the ratio of specific heats, $\Delta V$ is change in volume due to the displacement of the fluid in the swirler, $V_{\mathrm{pl}}$ is the volume of the plenum, and $p_{0, \mathrm{pl}}$ is the initial pressure in the plenum. This increase in pressure tends to push back on the fluid in the swirler, providing a spring effect. A damper is added to the spring-mass system to consider the entropy generated during compression. Note that an increase in pressure in the plenum would cause the same effect on the fluid in Cylinder 4. However, the the flow emanating from the premixer was choked, and therefore no disturbances could propagate upstream to cause mass flow oscillations. As a result, the premixer was transparent to acoustic oscillations while only the section of Cylinder 4 downstream of the choked flow, along with the plenum, chamber, and swirler must be considered in the acoustic modelling.

The system of equations representing the behaviour of the Helmholtz resonator can be established by looking at the balance of forces acting on the fluid mass $\left(m_{\mathrm{s}}\right)$ in the swirler. The Free Body Diagram (FBD) of $m_{\mathrm{s}}$ is shown in Fig. 4.20, and for any movement and acceleration in the positive $\xi$ direction, the sum of the inertial, viscous, and pressure 
forces are

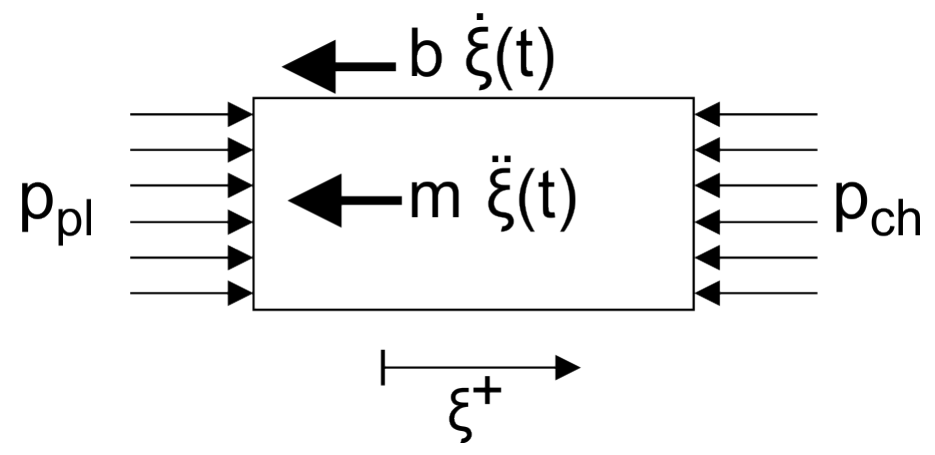

Figure 4.20: Free body diagram of the forces acting on $m_{\mathrm{s}}$ as it is accelerated in the positive $\xi$ direction.

$$
-m_{\mathrm{s}} \frac{\mathrm{d}^{2} \xi}{\mathrm{d} t^{2}}-b \frac{\mathrm{d} \xi}{\mathrm{d} t}-\left(p_{\mathrm{pl}}(t)-p_{\mathrm{ch}}(t)\right) S=0
$$

where $b$ is the linear damping constant at $m_{\mathrm{s}}$ and $S$ is the cross-section area of $m_{\mathrm{s}}$. The pressure in the plenum can be expressed as a function of the unperturbed value $\left(p_{\mathrm{pl}, 0}\right)$, the volume of the plenum $\left(V_{\mathrm{pl}}\right)$, and the volumetric displacement $\Delta V$ via Eq. 4.6, where the change in volume resulting from the displacement of $m_{\mathrm{s}}$ is

$$
\Delta V=\xi(t) S
$$

Substituting Eq. 4.6 and 4.8 into Eq. 4.7, the differential equation describing the state of $m_{\mathrm{s}}$ can be written as

$$
-m_{\mathrm{s}} \frac{\mathrm{d}^{2} \xi}{\mathrm{d} t^{2}}-b \frac{\mathrm{d} \xi}{\mathrm{d} t}-\left(-\frac{\gamma p_{\mathrm{pl}, 0}}{V_{\mathrm{pl}}} S x(t)-p_{\mathrm{ch}}(t)\right) S=0 .
$$

defining $k=-\frac{\gamma p_{\mathrm{pl}, 0}}{V_{\mathrm{pl}}} S$ for notational convenience, the equation simplifies to

$$
-m_{\mathrm{s}} \frac{\mathrm{d}^{2} \xi}{\mathrm{d} t^{2}}-b \frac{\mathrm{d} \xi}{\mathrm{d} t}-k S \xi-S p_{\mathrm{ch}}(t)=0
$$

Assuming a harmonic excitation and response of the form $p_{\mathrm{ch}}(t)=P_{\mathrm{ch}} \mathrm{e}^{i \omega t}$ and $\xi(t)=$ $\Xi \mathrm{e}^{i \omega t}, \mathrm{Eq} .4 .10$ can be expressed as a function of the angular frequency $(\omega)$ :

$$
m_{\mathrm{s}} \omega^{2} \Xi-b \omega i \Xi-k S \Xi-S P_{\mathrm{ch}}=0 \text {. }
$$

For given values of the constants $\left(m_{\mathrm{s}}, k\right.$, and $\left.b\right)$, the displacement amplitude $(\Xi)$ could then be expressed as a function of the forcing amplitude and frequency

$$
\Xi\left(\omega, P_{\mathrm{ch}}\right)=\frac{S P_{\mathrm{ch}}}{m_{\mathrm{s}} \omega^{2}-b \omega i-k S} .
$$




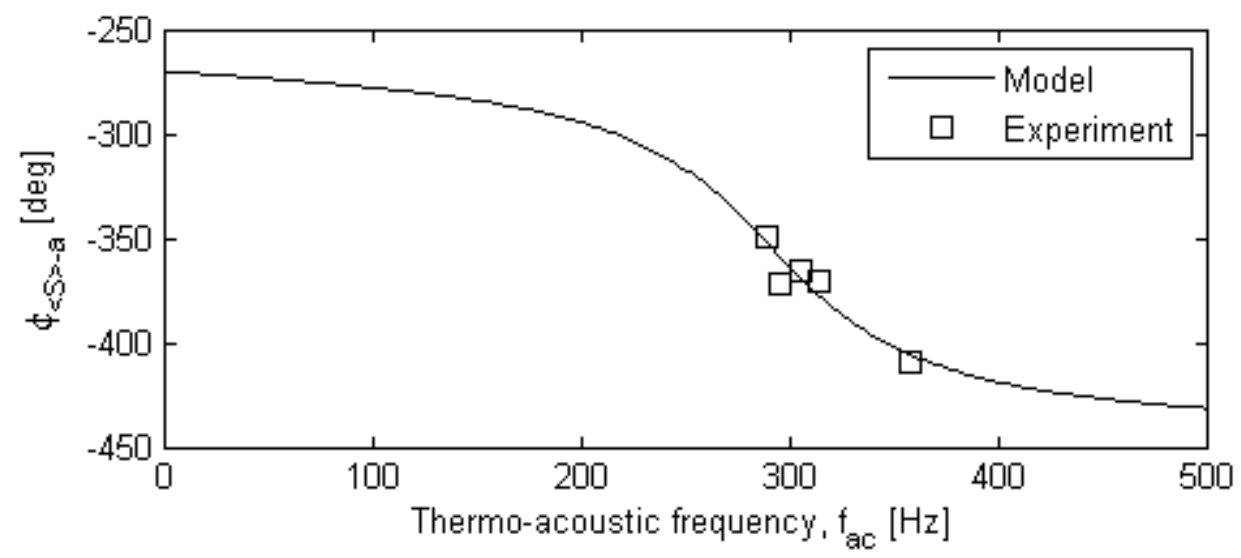

Figure 4.21: Phase lag of the reactant influx oscillations with respects to the chamber pressure as a function of acoustic forcing frequency. The Helmholtz resonator model (solid line) and experimental data (square markers) show good agreement, allowing for simple prediction of the space-phase distribution of the axial velocity wave $\left(\left\langle u_{\mathrm{y}}\right\rangle^{\mathrm{a}}\right)$.

The velocity fluctuations at the nozzle exit plane can be derived from this expression. Given that velocity is the temporal derivative position, the axial velocity fluctuations are given by:

$$
\begin{aligned}
\left\langle u_{\mathrm{y}}\right\rangle\left(\omega, P_{\mathrm{ch}}, y=0, \phi_{\mathrm{a}}\right) & =\frac{i \omega S P_{\mathrm{ch}}}{m_{\mathrm{s}} \omega^{2}-b \omega i-k S} \mathrm{e}^{i \omega \phi_{\mathrm{a}}} \\
& =U_{\mathrm{y}} \mathrm{e}^{i \omega \phi_{\mathrm{a}}}
\end{aligned}
$$

This equation can then be substituted into Eq. 4.5 to obtain the swirl number fluctuations at the nozzle exit. To verify the validity of this relation, the stiffness $(k)$, damping $(c)$, and inertial $(m)$ constants were computed by non-linear least squares curve fitting of finite elements simulation results generated by Seong [108]. These simulations presented the pressure ratio between the plenum and the chamber, a quantity that was independent of the chamber pressure amplitude, and therefore the amplitude of the swirling strength wave could not be verified. Instead, the relation was validated by computing $\phi_{<S>-a}$ via

$$
\phi_{\langle S\rangle^{\mathrm{a}}-\mathrm{a}}=\operatorname{atan}\left(\frac{\Im(U y)}{\Re(U y)}\right)+\pi,
$$

which was then compared to the experimental data. This comparison is shown in Fig. 4.21 , in which good agreement between the model and the experimental data shows that Helmoltz resonator modelling of the combustor is a useful tool for accurate prediction of the phase lag between the swirl number wave and the chamber acoustics. 


\section{Chapter 5}

\section{Conclusion}

This dissertation has provided new insight into flow-flame interactions leading to thermoacoustic coupling in a swirl-stabilized gas turbine model combustor via analysis of data from high-repetition-rate laser diagnostics. Furthermore, the nature of the coupling mechanism between the heat release oscillations, the velocity field, and the acoustics of the burner were identified.

The pressure in the gas turbine model combustor was found to be spatially uniform, and oscillated at a frequency different from that at which the HVC precessed around the burner. This allowed for statistics to be compiled as a function of both acoustic and HVC phases, and a statistical analogue to the Rayleigh integral was used to compute the thermo-acoustic energy transfer with the help of doubly-phase-resolved flame surface density and pressure. Most of the thermo-acoustic energy transfer originated from an outer coupling region, which surrounded the outer perimeter of the HVC. This was due to a periodic ignition of fresh reactants in the OCR, caused by the flame's oscillatory movement around the HVC, the cause of which was a deformation of the HVC over the thermo-acoustic cycle. This deformation was induced by a swirl number wave that

propagated from the burner nozzle as a result of the changing pressure difference between the combustor and the plenum. The speed at which this wave convected downstream was calculated, and corresponded to the mean axial velocity in the burner.

The variability in thermo-acoustic energy transfer with operating conditions such as thermal power was found to be the result of a change in phase lag between the heat release 
and pressure oscillations. Specifically, as thermal power increased, so did thermo-acoustic energy transfer. This increase was not associated with an increase in the amplitude of the heat release oscillations. Instead, the heat release oscillations remained roughly constant while the phase lag between the heat release and pressure oscillations decreased. To understand this phenomenon, the phase lag between the pressure and heat release oscillations was compared to the phase lag between the swirl number fluctuations at the nozzle exit plane and the acoustic cycle. The two phase relationships were almost identical, though the phase lag between the swirl and FSD fluctuations decreases slightly as a function of the operating conditions. This was due to an increase in the convective speed of the swirl number wave, which decreased the time necessary for the swirl number fluctuations at the nozzle exit plane to propagate downstream and deform the HVC, thereby decreasing the phase lag between the swirl number and FSD fluctuations.

The phase between the swirl number fluctuations at the nozzle exit and the acoustic cycle was further investigated with the help of a Helmholtz resonator model. This model was able to accurately predict the phase lag between the swirl number fluctuations and the acoustics. Note that this model is also capable of predicting the amplitude of the axial velocity wave, though amplitude information was not available for curve fitting of the model's constants and therefore was not studied here.

\section{$5.1 \quad$ Future work}

Despite the insight that this dissertation provides into the behaviour of flow-flame interactions in gas turbine combustor-like swirl-stabilized flames, achieving predictive capabilities for the limit-cycle amplitude of such flames will require further investigation. Assuming modelling will be achieved through the combined flame sheet and flame transfer function approach outlined in Sec. 1.4, quantitative prediction of the flow and flame dynamics is required. For the coupling mechanism outlined in this work, this entails quantitative modelling of the swirl number wave, the HVC's deformation, the long time average location of the flame as a function of the acoustic forcing frequency and amplitude, and general parameters related to operating conditions and geometry of the combustor.

The first topic was broached in Sec. 4.3.1, where a model capable of predicting both 
the phase and the amplitude of the swirl number wave was elaborated, making use of Helmholtz resonator theory. However, this model was shown to be adequate by curve fitting six different constants to finite element simulations performed by Seong [108]. In order to make this model truly predictive, these constants should be derived from the geometry of the combustor. While Helmholtz resonator theory provides rough estimates for these quantities [107], correction factors will need to be devised. For example, theory suggests that if the fluid in the throat sections moves uniformly, than the inertial constants $(m)$ should be

$$
m=\rho S l
$$

where $\rho$ is the density of the gas in the throat, $S$ is the cross-sectional area of the throat, and $l$ is its length. However, it is well known that fluids do not behave as a lumped mass, and therefore a correction factor must be introduced which depends on the geometry of the combustor. The same is true for the stiffness and damping constants.

Based on the phase and amplitude of the swirl number fluctuations, the deformation of the HVC must then be quantified. This will require a thorough understanding of the coupling between the velocity wave and the HVC. Moreover, the apparition of an HVC in the flow field should be predicted, and the effect of combustion and flow parameters on its precession frequency should be investigated. Once these are known, the HVC's deformation can be used in combination with the G-equation to predict the flame's movement, and associated heat release fluctuations. Of course, this will also require that the mean G-field, representative of the flame's location (see Sec. 1.4.2), be predicted from a set of relevant parameters.

The heat release oscillations could then be predicted as a function of the relevant flow and geometrical parameters, as well as the acoustic forcing frequency and amplitude. This information could be used to derive an FTF for identification of the unstable modes. Combined with an appropriate model for damping of thermo-acoustic energy, the limitcycle amplitude of a given combustor could be identified.

Achieving all of these goals may require higher phase resolutions, or lower statistical bias in the doubly phase resolved data. While increasing the number of phases decreases the uncertainty associated to phase resolution, it also decreases the number of measurements available for each phase, and therefore increases the statistical bias. Therefore, an increased number of measurements could prove useful in further analyses. For a 
description of uncertainty analysis of doubly-phase resolved statistics, see Ref. [73].

Finally, it is noted that all the experiments used for the analysis of this document were performed in a flame operating at atmospheric pressure, and in a can-type combustor. The reality of the problem is that gas turbine combustors typically operate at high pressures, and many modern gas turbine engines use annular or can-annular combustors. With compressor pressure ratios of approximately 40 and atmospheric pressures at flight altitude in the range $20-30 \mathrm{kPa}$, modern combustors operate at pressures that are an order of magnitude higher than those used in the current experiments. If the models discussed above are to be used in the early design stages of combustion chambers, then their validity should be verified at these higher pressures. Furthermore, combustor geometries with multiple inlets and exhausts must be studied in order to qualify the thermo-acoustic behaviour of annular and can-annular combustors, where the pressure, flame, and flow dynamics around a given injector are free to propagate and affect the dynamics of neighbouring injectors. 


\section{References}

[1] F. J. Verkamp, A. J. Verdouw, and J. G. Tomlinson. Impact of Emission Regulations on Future Gas Turbine Engine Combustors. J. Aircraft, 11, No.6, 340-344, 1974.

[2] S. K. Dhanuka. An Experimental Study of the Stable and Unstable Operation of an LPP Gas Turbine Combustor. PhD thesis, University of Michigan, 2008.

[3] H. S. Johnston. Chemistry in the stratosphere. Department of Transportation, Washington D.C., CIAP Monogr. 1, chap5, 1975.

[4] S. A. Mosier and R. M. Pierce. Advanced combustor systems for stationary gas turbine engines, phase I. Review and preliminary evaluation. U.S. Environmental Protection Agency, Vol 1., contract 68-02-2136, FR-11405, Finalr Report, 1980.

[5] A. S. Novick and D. L. Troth. Low $N O_{\mathrm{x}}$ heavy fuel combustor concept program. NASA CR-165367, 1981.

[6] H. G. Lew, D. B. Carl, G. Vermes, E. A. DeZubay, J. A. Schwab, and D. Prothroe. Low $N O_{\mathrm{x}}$ heavy fuel combustor concept program-phase 1: combustion technology generation. NASA CR-165482, 1981.

[7] G. Beal, B. Hinton, F. Kemp, P. Russel, and R. Sederquist. Low NO heavy fuel combustor concept program. NASA CR-165512, 1981.

[8] F. Kemp, R. Sederquist, and T. J. Rosfjord. Low $N O_{\mathrm{x}}$ heavy fuel combustor concept program-phase 1A: coal gas addendum final report. NASA CR-165577, 1982.

[9] F. Mao and R. B. Barat. Minimization of NO during staged combustion of $\mathrm{CH}_{3} \mathrm{NH}_{2}$. Combust. Flame, 105, 557-568, 1996. 
[10] R. J. Shaw, S. Gilkey, and R. Himes. Engine technology challenges for a 21st century high-speed civil transport. NASA TM-106216, 1993.

[11] T. J. Rosfjord and F. C. Pagent. Experimental assessment of the emissions control potential of a rich/quench/lean combustor for high speed civil transport aircraft engines. NASA CR-2001-210613, 2001.

[12] J. D. Holdeman, C. T. Chang, and H. L. Nguyen. Low emissions RQL flametube combustor component test results. NASA TM-2001-210678, 2001.

[13] G. S. Samuelsen, J. Brouwen, M. A. Vardakas, and J. D. Holdeman. Experimental and modeling investigation of the effect of air preheat on the formation of $N O_{\mathrm{x}}$ in an RQL combustor. Heat Mass Transfer, 49, 219-231, 2012.

[14] N. Docquier and S. Candel. Combustion control and sensors: a review. Prog. Energy Combust. Sci., 28, 107-150, 2002.

[15] I. Emiris and J. H. Whitelaw. Control of combustion oscillations. Combust. Sci. Tecnol., 175, 157-184, 2003.

[16] A. P. Dowling and A. S. Morgans. Annual Review of Fluid MechanicsRSS Feed. Annu. Rev. Fluid Mech., 37, 151-182, 2005.

[17] C. O. Paschereit and E. Gutmark. Active control of combustion instabilities in gas turbine burners. Proc. ASME Turbo Expo, No. GT2006-90186, 161-171, 2006.

[18] S. Ducruix, D. Durox, and S. Candel. Theoretical and Experimental Determinations of the Transfer Function of a Laminar Premixed Flame. Proc. Combust. Inst., 28, 765-773, 2000.

[19] P. Palies, D. Durox, T. Schuller, and S. Candel. The combined dynamics of swirler and turbulent premixed swirling flames. Combust. Flame, 157, 1698-1717, 2010.

[20] M. Lohrmann and H. Buchner. Prediction of Stability Limits for LP and LPP Gas Turbine Combustors. Combust. Sci. Technol., 177, 2243-2273, 2005.

[21] J. Ranalli, C. Martin, P. Black, U. Vandsburger, and R. West. Measurement of Flame Transfer Functions in SwirlStabilized, Lean-Premixed Combustion. J. Propul. Power, 25, 1350-1354, 2009. 
[22] D. You, Y. Huang, and V. Yang. A generalized model of acoustic response of turbulent premixed flame and its application to gas-turbine combustion instability analysis. Combust. Sci. and Tech., 177, 1109-1150, 2005.

[23] H. S. Preetham and T. Lieuwen. Dynamics of Laminar Premixed Flames Forced by Harmonic Velocity Disturbances. J. Propul. Power, 24, 1390-1402, 2008.

[24] T. Schuller, D. Durox, and S. Candel. A Unified Model for the Prediction of Laminar Flame Transfer Functions: Comparisons Between Conical and V-Flame Dynamics. Combust. Flame, 134, 21-34, 2003.

[25] S. Candel. Combustion dynamics and control: Progress and challenges. Proc. Combust. Inst., 29, P. 1-28, 2002.

[26] A. Bourehla and F. Baillot. Appearance and Stability of a Laminar Conical Premixed Flame Subjected to an Acoustic Perturbation. Combust. Flame, 114, 303-318, 1998.

[27] D. Durox, S. Ducruix, and S. Candel. Experiments on collapsing cylindrical flames. Combust. Flame, 125, 982-1000, 2001.

[28] S.-Y. Lee, S. Seo, J. C. Broda, S. Pal, and R. J. Santoro. An experimental estimation of mean reaction rate and flame structure during combustion instability in a lean premixed gas turbine combustor. Proc. Combust. Inst., 28, 775-782, 2000.

[29] V. G. McDonell and G. S. Samuelsen. Measurement of fuel mixing and transport processes in gas turbine combustion. Meas. Sci. Technol., 11, 870-886, 2000.

[30] Y. Deguchi, M. Noda, Y. Fukuda, Y. Ichinose, Y. Endo, M. Inada, Y. Abe, and S. Iwasaki. Industrial applications of temperature and species concentration monitoring using laser diagnostics. Meas. Sci. Technol., 13, R103-R115, 2002.

[31] P. Griebel, P. Siewert, and P. Jansohn. Flame characteristics of turbulent lean premixed methane/air flames at high pressure: Turbulent flame speed and flame brush thickness. Proc. Combust. Inst., 31, 3083-3090, 2007.

[32] U. Stopper, M. Aigner, W. Meier, R. Sadanandan, M. Stöhr, and I. K. Soo. Flow Field and Combustion Characterization of Premixed Gas Turbine Flames by Planar Laser Techniques. J. Eng. Gas Turb. Power, 131, 021504, 2009. 
[33] P. Strakey, S. d. Woodruff, T. C. Williams, and R. W. Schefer. OH-Planar Fluorescence Measurements of Pressurized, Hydrogen Premixed Flames in the SimVal Combustor. AIAA J., 46, 1604-1613, 2008.

[34] S. Dhanuka, J. E. Temme, J. F. Driscoll, and H. C. Mongia. Vortex-shedding and mixing layer effects on periodic flashback in a lean premixed prevaporized gas turbine combustor. Proc. Combust. Inst., 32, 2901-2908, 2009.

[35] R. Sadanandan, M Stöhr, and W. Meier. Simultaneous OH-PLIF and PIV measurements in a gas turbine model combustor. Appl. Phys. B., 90, 609-618, 2008.

[36] M Stöhr, R. Sadanandan, and W. Meier. Experimental study of unsteady flame structures of an oscillating swirl flame in a gas turbine model combustor. Proc. Combust. Inst., 32,2925-2932, 2009.

[37] F. L. Zwemer, D. S. Pratt, and J. J. May. Silo filler's disease in New York State. Am. Rev. Respir. Dis., 146, 650-653, 1992.

[38] J. Lepeule, F. Laden, D. Dockery, and J. Schwartz. Chronic Exposure to Fine Particles and Mortality: An Extended Follow-up of the Harvard Six Cities Study from 1974 to 2009. Environ. Health Persp., 120, 965-970, 2012.

[39] V. Ramaswamy. Radiative forcing of climate change. Climate Change, Cambrige Univ. Press, New York, 2001.

[40] M. Hori. Effects of Probing Conditions on NO2/NOx Ratios. Combust. Sci. Technol., 23, 131-135, 1980.

[41] J. C. Kramlich and P. C. Malte. Further Observations of the Effect of Sample Probes on Pollutant Gases Drawn from Flame Zones. Combust. Sci. Technol., 23, 262-269, 1980.

[42] C. Nickol. Environmentally Responsible Aviation (ERA) ProjectAssessing Progress Toward Simultaneous Reductions in Noise, Fuel Burn and NOx. NASA, $20110011739,2011$.

[43] E. Jahangir. ICAO Technology Goals Process for Aviation Environmental Protection. ICAO, 2010. 
[44] F. Collier. Environmentally Responsible Aviation Project: Real Solutions for Environmental Challenges Facing Aviation. AIAA ASM 2012, Nashville, 2012.

[45] International Civil Aviation Organization. ICAO Environmental Report. ICAO, 2010.

[46]

[47] J. A. Miller and C. T. Bowman. Mechanism and Modeling of Nitrogen Chemistry in Combustion. Prog. Energy Comb. Sci., 15, 287-388, 1989.

[48] S. M. Correa. A Review of $\mathrm{NO}_{\mathrm{x}}$ Formation Under Gas-Turbine Combustion Conditions. Combust. Sci. and Tech., 87, 329-362, 1992.

[49] M. C. Drake and J. B. Richard. Caluculations of NOx Formation Pathways in Propogating Laminar, High Pressure Premixed CH4/Air Flames. P. I. Mech. Eng. A-J Pow., 221, P. 387-398, 2007.

[50] T. Rutar, J. C. Y. Lee, P. Dagaut, P. C. Malte, and A. A. Bryne. NOx Formation Pathways in Lean-Premixed-Prevapourized Combustion of Fuels with Carbon-toHydrogen Ratios Between 0.25 and 0.88. P. I. Mech. Eng. A-J Pow., 221, P. 387-398, 2007.

[51] Y. Huang and V. Yang. Dynamics and stability of lean-premixed swirl-stabilized combustion. Prog. Energ. Combust. , 35, P. 293-364, 2009.

[52] T. C. Lieuwen and V. Yang. Combustion Instabilities in Gas Turbine Engines: Operational Experience, Fundamental Mechanics, and Modeling. AIAA, 2005.

[53] K. R. McManus, T. Poinsot, and S. M. Candel. A review of active control of combustion instabilities. Prog. Energy Comb., 19, 1-29, 1993.

[54] P. Palies, T. Schuller, D. Durox, and S. Candel. Modeling of premixed swirling flames transfer functions. Proc. Comb. Inst., 33, 2967-2974, 2011.

[55] B. Schuermans, F. Guethe, D. Pennel, D. Guyot, and C. Paschereit. Thermoacoustic modeling of a gas turbine using transfer functions measured at full engine pressure. ASME Turbo Expo 2009, GT2009-59605, 2009. 
[56] K. Kim, J. G. Lee, B. D. Quay, and D. A. Santavicca. Spatially distributed flame transfer functions for predicting combustion dynamics in lean premixed gas turbine combustors. Combust. Flame, 157, 1718-1730, 2010.

[57] C. J. Lawn. Thermo-acoustic frequency selection by swirled premixed flames. Proc. Combust. Inst., 28, 823-830, 2000.

[58] D. Durox, T. Schuller, and S. Candel. Combustion dynamics of inverted conical flames. Proc. Combust. Inst., 30, 1717-1724, 2005.

[59] R. Balachandran, B. Ayoola, C. Kaminski, A. Dowling, and E. Mastorakos. Experimental investigation of the nonlinear response of turbulent premixed flames to imposed inlet velocity oscillations. Combust. Flame, 143, 37-55, 2005.

[60] C. Kulsheimer and H. Buchner. Combustion dynamics of turbulent swirling flames. Combust. Flame, 131, 70-84, 2002.

[61] P. Palies, D. Durox, T. Schuller, and S. Candel. Nonlinear combustion instability analysis based on the flame describing function applied to turbulent premixed swirling flames. Combust. Flame, 158, 1980-1991, 2011.

[62] M. Fleifil, A. M. Annaswamy, Z. A. Ghoneim, and A. F. Ghoneim. Response of a Laminar Premixed Flame to Flow Oscillations: A Kinematic Model and Thermoacoustic Instability Results. Combust. Flame, 106, 487-510, 1996.

[63] T. Schuller, D. Durox, and S. Candel. Self-induced combustion oscillations of laminar premixed flames stabilized on annular burners. Combust. Flame, 135, 525-537, 2003.

[64] T. Lieuwen. Nonlinear kinematic response of premixed flames to harmonic velocity disturbances. Proc. Combust. Inst., 30, 1725-1732, 2005.

[65] J. A. Ranalli, D. Ferguson, and C. Martin. Simple Analysis of Flame Dynamics via Flexible Convected Disturbance Models. J. Propul. Power, 28, 1268-1276, 2012.

[66] F. Baillot, D. Durox, and R. Prud'homme. Experimental and Theoretical Study of a Premixed Vibrating Flame. Combust. Flame, 88, 149-168, 1992.

[67] L. Boyer and J. Quinard. On the Dynamics of Anchored Flames. Combust. Flame, 82, 51-65, 1990. 
[68] G. Borghesi, F. Biagioli, and B. Schuermans. Dynamic response of turbulent swirling flames to acoustic perturbations. Combust. Theory Modell., 13, 487-512, 2009.

[69] T. Komarek and W. Polifke. Impace of Swirl Fluctuations on the Flame Response of a Pervectly Premixed Swirl Burner. J. Eng. Gas Turbines Power , 132, 061503, 2010.

[70] I. Glassman and R. A. Yetter. Combustion, $4^{\text {th }}$ edition. Academic Press, 417-441, 2008.

[71] N. Syred. A review of oscillation mechanisms and the role of the precessing vortex core (PVC) in swirl combustion systems. Prog. Energ. Combust., 32, 93-161, 2006.

[72] C. O. U. Umeh. Theoretical and experimental studies of vortex breakdown in a lean, premixed swirl-stabilized combustor. Dissertation, 2009.

[73] A. M. Steinberg, I. Boxx, M. Stöhr, W. Meier, and C. D. Carter. Effects of flow structure dynamics on thermo-acoustic instabilities in swirl stabilized combustion. AIAA, 50, 952-975, 2011.

[74] Y. Sommerer, D. Galley, t. Poinsot, S. Ducruix, F. Lacas, and D. Veynante. Lare eddy simulation and experimental study of flashback and blow-off in a lean partially premixed swirled burner. J. Turbul., 5, 1468-5248, 2004.

[75] F. F. Grinstein and C. Fureby. LES studies of the flow in a swirl gas combustor. Proc. Combust. Inst., 30, 1791-1798, 2005.

[76] S. Menon and N. Patel. Subgrid Modeling for Simulation of Spray Combustion in Large-Scale Combustors. AIAA J., 44, 709-723, 2006.

[77] H. Pitsch. Large-Eddy Simulation of Turbulent Combustion. Ann. Rev. Fluid Mech., 38, 453-482, 2006.

[78] J. C. Oefelein. Large eddy simulation of turbulent combustion processes in propuslion and power systems. Prog. Aerosp. Sci., 42, 2-37, 2006.

[79] K. Kohse-Höinghaus. Laser techniques for the quantitative detection of reaction intermediates in combustion systems. Prog. Energ. Combust., 20, 203-279, 1994. 
[80] P. P. Radi, P. Beaud, H.-M. Frey, T. Gerber, B. Mischler, and A.-P. Tzannis. Analysis of Radicals in Combustion Processes. Chimia, 51,771-776, 1997.

[81] E. P. Hassel and S. Linow. Laser diagnostics for studies of turbulent combustion. Meas. Sci. Technol., 11, R37-R56, 2000.

[82] J.-M. Samaniego, F. N. Egolfopoulos, and C. T. Bowman. CO2 Chemiluminescence in Premixed Flames. Combust. Sci. and Tech., 109, 183-203, 1995.

[83] I. Grant. Particle imagery velocimetry: A review. J. Mech. Eng. Sci., 211, 1997.

[84] R. J. Adrian. 20 Years of Particle Image Velocimetry. Exp. in Fluids, 39, 159-169, 2005.

[85] W. Meier, P. Weigand, X. Duan, and R. Giezendanner-Thoben. Detailed characterization of the dynamics of thermoacoustic pulsations in a lean premixed swirl flame. Combust. Flame, 150, 2-23, 2007.

[86] P. Weigand, W. Meier, X. R. Duan, and M. Aigner. Laser-based investigations of thermoacoustic instabilities in a lean premixed gas turbine model combustor. J. Eng. Gas Turb. Power, 129, 664-671, 2007.

[87] W. Meier, I. Boxx, M. Stöhr, and C. D. Carter. Laser-based investigations in gas turbine model combustors. Exp. Fluids, 49, 865-882, 2010.

[88] C. M. Arndt, A. M. Steinberg, I. Boxx, W. Meier, and M. Aigner. Flow-field and flame dynamics of a gas turbine model combustor during transition between thermo-acoustically stable and unstable states. Proceedings of ASME Turbo Expo Glasgow, UK, GT2010-22830, 2010.

[89] S. Roux, G. Lartigue, T. Poinsot, U. Meier, and C. Bérat. Studies of mean and unsteady flow in a swirled combustor using experiments, acoustic analysis and large eddy simulations. Combust. Flame, 141, 40-54, 2005.

[90] J. Galpin, A. Naudin, L. Vervisch, C. Angelberger, O. Colin, and P. Domingo. Large-eddy simulation of a fuel-lean premixed turbulent swirl-burner. Combust. Flame, 155, 247-266, 2008. 
[91] B. Franzelli, E. Riber, L. Y. M. Gicquel, and T. Poinsot. Large eddy simulation of combustion instabilities in a lean partially premixed swirled flame. Combust. Flame, 159, 621-637, 2012.

[92] P. Weigand, W. Meier, X. R. Duan, W. Stricker, and M. Aigner. Investigations of swirl flames in a gas turbine model combustor I. Flow field, structures, temperature, and species distribution. Combust. Flame, 144, 205-224, 2006.

[93] W. Meier, X. R. Duan, and P. Weigand. Investigations of swirl flames in a gas turbine model combustor II. Turbulence-chemistry interactions. Combust. Flame, 144, 225-236, 2006.

[94] I. Boxx, M. Stöhr, C. Carter, and W. Meier. Temporally resolved planar measurements of transient phenomena in a partially premixed swirl flame in a gas turbine model combustor. Combust. Flame, 157, 1510-1525, 2010.

[95] M. Stöhr, I. Boxx, C. Carter, and W. Meier. Dynamics of lean blowout of a swirlstabilized flame in a gas turbine model combustor. Proc. Combust. Inst., 33, 16631672, 2011.

[96] A. M. Steinberg, C. M. Arndt, and W. Meier. Parametric study of vortex structures and their dynamics in swirl-stabilized combustion. Proc. Combust. Inst., 34, 3117$3125,2013$.

[97] H. J. Lugt. The Dilemma of Defining a Vortex. Recent Developments in Theoretical and Experimental Fluid Mechanics, A79-29651 11-34, 309-321, 1979.

[98] J. Jeong and F. Hussain. On the identification of a vortex. J. Fluid Mech., 285, 69-94, 1995.

[99] V. Kolar. Vortex identification: New requirements and limitations. Int. J. Heat Fluid Fl., 28, 638-652, 2007.

[100] C.-T. Pan and K. Sigmon. A Bottom-Up Inductive Proof of the Singular Value Decomposition. Recent Developments in Theoretical and Experimental Fluid Mechanics, A79-29651 11-34, 309-321, 1979. 
[101] A. M. Steinberg, I. Boxx, M. Stöhr, C. D. Carter, and W. Meier. Flow-flame interactions causing acoustically coupled heat release fluctuations in a thermo-acoustically unstable gas turbine model combustor. Combust. Flame,157, 2250-2266, 2010.

[102] C. Kortschik, T. Plessing, and N. Peters. Laser optical investigation of turbulent transport of temperature ahead of the preheat zone in a premixed flame. Combust. Flame,136, 43-50, 2004.

[103] R. Knicker, D. Veynante, J. C. Rolon, and C. Meneveau. Planar laser-induced fluorescence in a turbulent premixed flame to analyze large eddy simulation models. Heat Mass Trans., 2000.

[104] D. S. Louch. A Different Physical Interpretation of Flame Sheet Surface Density. Combust. Sci. and Tech.,108, 81-90, 1995.

[105] I. G. Shepherd. Flame surface density and burning rate in premixed turbulent flames. $25^{\text {th }}$ Combustion Symposium, 373-379, 1996.

[106] P. Palies, T. Schuller, D. Durox, L. Y. M. Gicquel, and S. Candel. Acoustically perturbed turbulent premixed swirling flames. Phys. Fluids, 23, 037101, 2011.

[107] L. E. Kinsler and A. R. Frey. Fundamentals of Acoustics. John Wiley \& Sons, New York, 1962.

[108] H. I. Seong. Caratérisation des instabilités thermoacoustiques dans les chambres de combustion à gaz. Dissertation, École Centrale de Paris, 2012. 